\title{
Self-duality of the compactified Ruijsenaars-Schneider system from quasi-Hamiltonian reduction
}

\author{
L. Fehér ${ }^{a}$ and C. Klimčík ${ }^{b}$ \\ ${ }^{a}$ Department of Theoretical Physics, WIGNER RCP, RMKI \\ H-1525 Budapest, P.O.B. 49, Hungary, and \\ Department of Theoretical Physics, University of Szeged \\ Tisza Lajos krt 84-86, H-6720 Szeged, Hungary \\ e-mail: lfeher@rmki.kfki.hu \\ ${ }^{b}$ Institut de mathématiques de Luminy \\ 163, Avenue de Luminy \\ F-13288 Marseille, France \\ e-mail: klimcik@univmed.fr
}

\begin{abstract}
The Delzant theorem of symplectic topology is used to derive the completely integrable compactified Ruijsenaars-Schneider III $_{\mathrm{b}}$ system from a quasi-Hamiltonian reduction of the internally fused double $S U(n) \times S U(n)$. In particular, the reduced spectral functions depending respectively on the first and second $S U(n)$ factor of the double engender two toric moment maps on the $\mathrm{III}_{\mathrm{b}}$ phase space $\mathbb{C} P(n-1)$ that play the roles of action-variables and particle-positions. A suitable central extension of the $S L(2, \mathbb{Z})$ mapping class group of the torus with one boundary component is shown to act on the quasi-Hamiltonian double by automorphisms and, upon reduction, the standard generator $S$ of the mapping class group is proved to descend to the Ruijsenaars self-duality symplectomorphism that exchanges the toric moment maps. We give also two new presentations of this duality map: one as the composition of two Delzant symplectomorphisms and the other as the composition of three Dehn twist symplectomorphisms realized by Goldman twist flows. Through the well-known relation between quasi-Hamiltonian manifolds and moduli spaces, our results rigorously establish the validity of the interpretation [going back to Gorsky and Nekrasov] of the $\mathrm{III}_{\mathrm{b}}$ system in terms of flat $S U(n)$ connections on the one-holed torus.
\end{abstract}




\section{Contents}

1 Introduction

2 Preliminaries

2.1 Quasi-Hamiltonian systems and their reductions . . . . . . . . . . 6

2.2 Evolution flows on the internally fused double of $S U(n) \ldots \ldots \ldots$

3 Reduction of the internally fused double of $S U(n) \quad \mathbf{1 1}$

3.1 The reduced phase space is smooth and compact . . . . . . . . . . 12

3.2 The images of the Hamiltonians $\alpha_{j}, \beta_{j}$ restricted to $\mu^{-1}\left(\mu_{0}\right) \ldots \ldots$. . . . . . 13

3.3 The reduced phase space is a Hamiltonian toric manifold . . . . . . . . . . . 19

3.4 The global structure of the reduced systems $(P, \hat{\omega}, \hat{\alpha})$ and $(P, \hat{\omega}, \hat{\beta}) \ldots \ldots 22$

4 Construction of the Delzant symplectomorphisms 25

4.1 Local version of the Delzant map $f_{\beta} \ldots \ldots \ldots$. . . . . . . . . . 25

4.2 Global extension of the Lax matrix . . . . . . . . . . . . . . . . . 31

4.3 Construction of the global Delzant map $f_{\beta} \ldots \ldots \ldots$. . . . . . . . . . . . . . .

4.4 The global Delzant map $f_{\alpha}$ and involution properties . . . . . . . . . . . 37

5 Self-duality of the compactified $\mathrm{III}_{\mathrm{b}}$ system 41

6 Duality and the mapping class group $\quad 45$

6.1 Flat connections and the quasi-Hamiltonian double . . . . . . . . . 46

6.2 Mapping class group of the one-holed torus . . . . . . . . . . . . . . 47

6.3 The duality map $\mathfrak{S}$ as a mapping class symplectomorphism . . . . . . . . . . 49

6.4 The anti-symplectomorphism $\mathfrak{R}$ as a $G L(2, \mathbb{Z})$ generator . . . . . . . . . . 51

\begin{tabular}{llr}
7 & Discussion & 52 \\
\hline
\end{tabular} 


\section{Introduction}

A remarkable feature of the non-relativistic [3, 34, 22] and relativistic [33] integrable many-body systems of Calogero type is their duality relation discovered by Ruijsenaars [29]. The phase space of a classical integrable many-body system is always equipped with two Abelian algebras of distinguished observables: the particle-positions tied to the physical interpretation and the action-variables tied to the Liouville integrability. The 'Ruijsenaars duality' between systems (i) and (ii) requires the existence of a symplectomorphism between the pertinent phase spaces that converts the particle-positions of system (i) into the action-variables of system (ii), and vice versa. One talks of self-duality if the leading Hamiltonians of systems (i) and (ii), which underlie the many-body interpretation, have the same form possibly with different coupling constants. In addition to being fascinating in itself, the duality proved useful for studying the dynamics, and it also appears at the quantum mechanical level where its manifestation is the bispectral property [6] of the many-body Hamiltonian operators [30, 32].

The duality relation has been established in [29, 31] with the help of a direct method for all non-elliptic Calogero type systems associated with the $A_{n}$ root system. The present paper is part of the series [8, 9, 7] aimed at understanding all Ruijsenaars dualities by means of the reduction approach. The basic tenet of this approach, which originated from the pioneering papers [27, 17], is that the integrable many-body systems descend from certain natural 'free' systems that can be reduced using their large symmetries. Regarding the duality, it is envisioned [10] that the starting phase spaces to be reduced actually carry two 'free' systems that turn into a dual pair in terms of two models of a single reduced phase space. The existence of the symplectomorphism between two models (that arise in the simplest cases as two gauge slices) of a single reduced phase space is entirely automatic. In this way the reduction approach may yield considerable technical simplification over the direct method, where the proof of the symplectic character of the duality map is very laborious. However, nothing guarantees that this approach must always work; finding the correct reduction procedure relies on inspiration.

To this date, the reduction approach has been successfully implemented for describing all but two cases of the known Ruijsenaars dualities. The remaining two cases are the self-dualities of the hyperbolic and of the compactified trigonometric Ruijsenaars-Schneider systems. The physical interpretation of the latter system (also called $\mathrm{III}_{\mathrm{b}}$ system in [31], with 'b' for 'bounded') is based on its local description valid before compactification. Since it is needed subsequently, next we briefly summarize this local description.

The definition of the III $_{\mathrm{b}}$ system begins with the local Hamiltonian 1

$$
H_{y}^{\mathrm{loc}}(\delta, \Theta) \equiv \sum_{j=1}^{n} \cos p_{j} \prod_{k \neq j}^{n}\left[1-\frac{\sin ^{2} y}{\sin ^{2}\left(x_{j}-x_{k}\right)}\right]^{\frac{1}{2}},
$$

where $y$ is a real non-vanishing parameter, the $\delta_{j}=e^{\mathrm{i} 2 x_{j}}(j=1, \ldots, n)$ are interpreted as the positions of $n$ 'particles' moving on the circle, and the canonically conjugate momenta $p_{j}$ encode the compact variables $\Theta_{j}=e^{-\mathrm{i} p_{j}}$. Here, the center of mass condition $\prod_{j=1}^{n} \delta_{j}=\prod_{j=1}^{n} \Theta_{j}=1$ is also adopted. Denoting the standard maximal torus of $S U(n)$ as $S \mathbb{T}_{n}$, the local phase space is

$$
M_{y}^{\mathrm{loc}} \equiv\left\{(\delta, \Theta) \mid \delta=\left(\delta_{1}, \ldots, \delta_{n}\right) \in \mathcal{D}_{y}, \Theta=\left(\Theta_{1}, \ldots, \Theta_{n}\right) \in S \mathbb{T}_{n}\right\}
$$

\footnotetext{
${ }^{1}$ The index $k$ in the next product $\prod_{k \neq j}^{n}$ runs over $\{1,2, \ldots, n\} \backslash\{j\}$, and similar notation is used throughout.
} 
where the domain $\mathcal{D}_{y} \subset S \mathbb{T}_{n}$ (a so-called Weyl alcove with thick walls [35]) is chosen in such a way to guarantee that $H_{y}^{\text {loc }}$ takes real values. The non-emptiness of $\mathcal{D}_{y}$ is ensured by the restriction $|y|<\frac{\pi}{n}$. The symplectic form on $M_{y}^{\text {loc }}$ reads

$$
\Omega^{\mathrm{loc}} \equiv \frac{1}{2} \operatorname{tr}\left(\delta^{-1} d \delta \wedge \Theta^{-1} d \Theta\right)=\sum_{j=1}^{n} d x_{j} \wedge d p_{j} .
$$

The Hamiltonian $H_{y}^{\text {loc }}$ can be recast as the real part of the trace of the unitary Lax matrix $L_{y}^{\text {loc }}$ :

$$
L_{y}^{\mathrm{loc}}(\delta, \Theta)_{j l} \equiv \frac{e^{\mathrm{i} y}-e^{-\mathrm{i} y}}{e^{\mathrm{i} y} \delta_{j} \delta_{l}^{-1}-e^{-\mathrm{i} y}} W_{j}(\delta, y) W_{l}(\delta,-y) \Theta_{l}
$$

with the positive functions

$$
W_{j}(\delta, y):=\prod_{k \neq j}^{n}\left[\frac{e^{\mathrm{i} y} \delta_{j}-e^{-\mathrm{i} y} \delta_{k}}{\delta_{j}-\delta_{k}}\right]^{\frac{1}{2}}
$$

The flows generated by the spectral invariants of $L_{y}^{\text {loc }}$ commute, but are not complete on $M_{y}^{\text {loc }}$. Ruijsenaars [31] has shown that one can realize $\left(M_{y}^{\text {loc }}, \Omega^{\text {loc }}\right)$ as a dense open submanifold of the complex projective space $\mathbb{C} P(n-1)$ equipped with a multiple of the Fubini-Study symplectic form, and thereby the commuting local flows generated by $L_{y}^{\text {loc }}$ extend to complete Hamiltonian flows on the compact phase space $\mathbb{C} P(n-1)$. The self-duality of the resulting compactified $\mathrm{III}_{\mathrm{b}}$ system was also proved in [31].

Besides its appearance in soliton theory, the importance of the $\mathrm{III}_{\mathrm{b}}$ system resides mainly in its interpretation in terms of an appropriate symplectic reduction of the space of $S U(n)$ connections on the torus with one boundary component (i.e. the one-holed torus). In fact, the local version of the $\mathrm{III}_{\mathrm{b}}$ system had been derived by means of such a symplectic reduction by Gorsky and his collaborators [14, 10] who moreover conjectured that the Ruijsenaars duality originates from the geometrically natural action of the $S L(2, \mathbb{Z})$ mapping class group of the torus on the reduced phase space. However, important global issues such as the compactification of the local phase space and the problem of the completeness of the Liouville flows were not addressed in their approach, and they have not proved that the Ruijsenaars self-duality symplectomorphism of [31] indeed originates from the action of the standard mapping class generator $S \in S L(2, \mathbb{Z})$.

The principal achievement of the present paper is a complete, global reduction treatment of the compactified $\mathrm{III}_{\mathrm{b}}$ system including a simple proof of its self-duality. The self-duality map will automatically arise as the composition of two Delzant symplectomorphisms, which will pave the way to also prove its conjectured relation [10] to the mapping class group. To obtain these results, we do not proceed by developing further the infinite-dimensional approach of [14], but shall rather work in a suitable finite-dimensional framework based on a non-trivial generalization of the Marsden-Weinstein symplectic reduction, called quasi-Hamiltonian reduction [1].

The quasi-Hamiltonian reduction was invented [1] as a finite-dimensional alternative for describing the symplectic structures on various moduli spaces of flat connections on Riemann surfaces whose investigation was initiated by Atiyah and Bott in the infinite-dimensional reduction context (see e.g. the book [18] and references therein). From this angle, it is not surprising 
that quasi-Hamiltonian methods can be applied for finite-dimensional reduction treatment of integrable systems 2 . Nevertheless, we find it remarkable how naturally the quasi-Hamiltonian geometry together with the Delzant theorem of symplectic topology [5] lead to an understanding of the global structure of the compactified Ruijsenaars-Schneider system. In fact, the Delzant theorem will be applied to establish the existence of two suitably equivariant symplectomorphisms, $f_{\alpha}$ and $f_{\beta}$, that both map $\mathbb{C} P(n-1)$ onto the quasi-Hamiltonian reduced phase space. By utilizing their main features, we also will be able to construct these Delzant symplectomorphisms explicitly, and then shall recover the Ruijsenaars self-duality symplectomorphism as the composition $\mathfrak{S}=f_{\alpha}^{-1} \circ f_{\beta}: \mathbb{C} P(n-1) \rightarrow \mathbb{C} P(n-1)$. A re-phrasing of this formula will allow us to interpret the self-duality of the compactified Ruijsenaars-Schneider system as a direct consequence of the 'mapping class democracy' between the $S U(n)$ factors of the quasiHamiltonian double. In fact, we shall prove the presentation $\mathfrak{S}=f_{\beta}^{-1} \circ S_{P} \circ f_{\beta}$ where $S_{P}$ stands for the natural action of $S \in S L(2, \mathbb{Z})$ on the quasi-Hamiltonian reduced phase space. Inspired by results of Goldman [12], $S_{P}$ itself will be decomposed into a product of three Dehn twist sympletomorphisms realized as special cases of certain Hamiltonian flows.

The paper is essentially self-contained and its organization is as follows. In Section 2, we first recall the concept of quasi-Hamiltonian dynamics and the method of quasi-Hamiltonian reduction. Then we describe the internally fused quasi-Hamiltonian double of the group $S U(n)$ and define two torus actions on it that will descend to the reduced phase space of our interest. In Section 3, we perform the reduction, we prove that the reduced phase space is a Hamiltonian toric manifold (in two alternative but equivalent ways) and we find its topology and symplectic structure by identifying the Delzant polytope corresponding to the moment map of the torus action. In Section 4 , we construct the Delzant symplectomorphisms $f_{\alpha}$ and $f_{\beta}$ explicitly. The local Lax matrix (1.4) and its global extension will arise naturally as building blocks of these maps. In Section 5, we recover the compactified Ruijsenaars-Schneider system and its selfduality from our reduction. In Section 6, we demonstrate that the action of the Ruijsenaars self-duality symplectomorphism on the $\mathrm{III}_{\mathrm{b}}$ phase space is the standard action of the mapping class generator $S \in S L(2, \mathbb{Z})$. Theorems 7,8 of Section 5 and Theorem 9 of Section 6 are our main results representing the final outcome of our analysis. Their implications are further discussed in Section 7, together with an outlook on open problems.

\section{Preliminaries}

Quasi-Hamiltonian systems can be useful since they can be reduced to honest Hamiltonian systems by a generalization of the standard Marsden-Weinstein reduction procedure, and this can give an effective tool for studying the resulting reduced systems. Below we first recall from [1] the relevant notions, and then describe those quasi-Hamiltonian dynamical systems that later will be shown to yield the compactified Ruijsenaars-Schneider system upon reduction.

\footnotetext{
${ }^{2}$ It was remarked by Oblomkov [26] that the Fock-Rosly treatment 11] of the complexified trigonometric Ruijsenaars-Schneider system could be replaced by quasi-Hamiltonian reduction based on $G L(n, \mathbb{C})$. This is very close in spirit to our framework, but the compact case that we consider is very different technically.
} 


\subsection{Quasi-Hamiltonian systems and their reductions}

Let $G$ be a compact Lie group with Lie algebra $\mathcal{G}$. Fix an invariant scalar product $\langle\cdot, \cdot\rangle$ on $\mathcal{G}$ and denote by $\vartheta$ and $\bar{\vartheta}$, respectively, the left- and right-invariant Maurer-Cartan forms on $G$. For a $G$-manifold $M$ with action $\Psi: G \times M \rightarrow M$, we use $\Psi_{g}(m):=\Psi(g, m)$ and let $\zeta_{M}$ denote the vector field on $M$ that corresponds to $\zeta \in \mathcal{G}$; we have $\left[\zeta_{M}, \eta_{M}\right]=-[\zeta, \eta]_{M}$ for all $\zeta, \eta \in \mathcal{G}$. The adjoint action of $G$ on itself is given by $\operatorname{Ad}_{g}(x):=g x g^{-1}$, and $\operatorname{Ad}_{g}$ denotes also the induced action on $\mathcal{G}$.

By definition [1], a quasi-Hamiltonian $G$-space $(M, G, \omega, \mu)$ is a $G$-manifold $M$ equipped with an invariant 2-form $\omega \in \Lambda(M)^{G}$ and with an equivariant map $\mu: M \rightarrow G, \mu \circ \Psi_{g}=\operatorname{Ad}_{g} \circ \mu$, in such way that the following conditions hold.

(a1) The differential of $\omega$ is given by

$$
d \omega=-\frac{1}{12} \mu^{*}\langle\vartheta,[\vartheta, \vartheta]\rangle .
$$

(a2) The infinitesimal action is related to $\mu$ and $\omega$ by

$$
\omega\left(\zeta_{M}, \cdot\right)=\frac{1}{2} \mu^{*}\langle\vartheta+\bar{\vartheta}, \zeta\rangle, \quad \forall \zeta \in \mathcal{G} .
$$

(a3) At each $x \in M$, the kernel of $\omega_{x}$ is provided by

$$
\operatorname{Ker}\left(\omega_{x}\right)=\left\{\zeta_{M}(x) \mid \zeta \in \operatorname{Ker}\left(\operatorname{Ad}_{\mu(x)}+\operatorname{Id}_{\mathcal{G}}\right)\right\}
$$

The map $\mu$ is called the moment map.

A quasi-Hamiltonian dynamical system $(M, G, \omega, \mu, h)$ is a quasi-Hamiltonian $G$-space with a distinguished $G$-invariant function $h \in C^{\infty}(M)^{G}$, the Hamiltonian. It follows from the axioms that there exists a unique vector field $v_{h}$ on $M$ determined by the following two requirements:

$$
\omega\left(v_{h}, \cdot\right)=d h, \quad \mathcal{L}_{v_{h}} \mu=0 .
$$

The 'quasi-Hamiltonian vector field' $v_{h}$ is $G$-invariant and it preserves $\omega, \mathcal{L}_{v_{h}} \omega=0$. Thus, $G$-invariant Hamiltonians on a quasi-Hamiltonian $G$-space define evolution flows in much the same way as arbitrary Hamiltonians do on symplectic manifolds. One can also introduce an honest Poisson bracket on $C^{\infty}(M)^{G}$. Naturally, if $f$ and $h$ are $G$-invariant functions and $v_{f}$ and $v_{h}$ the corresponding quasi-Hamiltonian vector fields, then this Poisson bracket is given by

$$
\{f, h\}:=\omega\left(v_{f}, v_{h}\right) .
$$

Indeed, it is not difficult to check that the result $\{f, h\}$ is again an invariant function and all the usual properties (including the Jacobi identity) are verified by this Poisson bracket. It is worth emphasizing that the general quasi-Hamiltonian manifold $M$ is not symplectic and the quasi-Hamiltonian form $\omega$ does not induce a proper Poisson algebra on the smooth functions on $M$ but just on the $G$-invariant smooth functions.

The quasi-Hamiltonian reduction of a quasi-Hamiltonian dynamical system $(M, G, \omega, \mu, h)$ that interests us is determined by choosing an element $\mu_{0} \in G$. We say that $\mu_{0}$ is strongly regular if it satisfies the following two conditions: 
1. The subset $\mu^{-1}\left(\mu_{0}\right):=\left\{x \in M \mid \mu(x)=\mu_{0}\right\}$ is an embedded submanifold of $M$.

2. If $G_{0} \subset G$ is the isotropy group of $\mu_{0}$ with respect to the adjoint action, then the quotient $\mu^{-1}\left(\mu_{0}\right) / G_{0}$ is a manifold for which the canonical projection $p: \mu^{-1}\left(\mu_{0}\right) \rightarrow \mu^{-1}\left(\mu_{0}\right) / G_{0}$ is a smooth submersion.

The result of the reduction based on a strongly regular element $\mu_{0}$ is a standard Hamiltonian system, $(P, \hat{\omega}, \hat{h})$. The reduced phase space $P$ is the manifold

$$
P \equiv \mu^{-1}\left(\mu_{0}\right) / G_{0},
$$

which carries the reduced symplectic form $\hat{\omega}$ and reduced Hamiltonian $\hat{h}$ uniquely defined by

$$
p^{*} \hat{\omega}=\iota^{*} \omega, \quad p^{*} \hat{h}=\iota^{*} h
$$

where $\iota: \mu^{-1}\left(\mu_{0}\right) \rightarrow M$ is the tautological embedding.

We stress that $\hat{\omega}$ is a symplectic form in the usual sense, whilst $\omega$ is neither closed nor globally non-degenerate in general. It follows from the above definitions that the Hamiltonian vector field and the flow defined by $\hat{h}$ on $P$ can be obtained by first restricting the quasi-Hamiltonian vector field $v_{h}$ and its flow to the 'constraint surface' $\mu^{-1}\left(\mu_{0}\right)$ and then applying the canonical projection $p$. The Poisson brackets on $(P, \hat{\omega})$ are inherited from the Poisson brackets (2.5) of the $G$-invariant functions as in standard symplectic reduction.

\subsection{Evolution flows on the internally fused double of $S U(n)$}

Consider a quasi-Hamiltonian space $M$ and a set of $k$ distinguished $G$-invariant functions on it. In the sense of the preceding subsection, these data define a family of quasi-Hamiltonian dynamical systems. We shall speak about a 'commuting $k$-family' if the corresponding quasiHamiltonian vector fields all commute among each other.

In this paper, we shall deal with two commuting $(n-1)$-families of quasi-Hamiltonian dynamical systems, which both live on a single quasi-Hamiltonian $G$-space. The quasi-Hamiltonian $G$-space in question is the so-called internally fused double of the group $G:=S U(n)$ [1], which as a manifold is provided by the direct product

$$
D:=G \times G=\{(A, B) \mid A, B \in G\} .
$$

The invariant scalar product on $\mathcal{G}:=s u(n)$ is given by

$$
\langle\eta, \zeta\rangle:=-\frac{1}{2} \operatorname{tr}(\eta \zeta), \quad \forall \eta, \zeta \in \mathcal{G}
$$

The group $G$ acts on $D$ by componentwise conjugation 3

$$
\Psi_{g}:(A, B) \mapsto\left(g A g^{-1}, g B g^{-1}\right) .
$$

\footnotetext{
${ }^{3}$ Later in some equations we apply $g \in U(n)$ in the formula of the action, which is harmless since only the factor group $S U(n) / \mathbb{Z}_{n} \simeq U(n) / U(1)$ acts effectively.
} 
The 2-form $\omega$ of $M:=D$ reads

$$
2 \omega=\left\langle A^{-1} d A^{\wedge}, d B B^{-1}\right\rangle+\left\langle d A A^{-1} \hat{,} B^{-1} d B\right\rangle-\left\langle(A B)^{-1} d(A B) \wedge \hat{\imath}(B A)^{-1} d(B A)\right\rangle,
$$

and the $G$-valued moment map $\mu$ is defined by

$$
\mu(A, B)=A B A^{-1} B^{-1}
$$

Consider a real class function $h \in C^{\infty}(G)^{G}$. Define the derivative $\nabla h \in C^{\infty}(G, \mathcal{G})^{G}$ by the equation

$$
\left.\frac{d}{d t}\right|_{t=0} h\left(e^{t \zeta} g\right)=\langle\zeta, \nabla h(g)\rangle, \quad \forall g \in G, \forall \zeta \in \mathcal{G}
$$

Associate to $h$ the following $G$-invariant functions on $D$,

$$
h_{1}(A, B):=h(A), \quad h_{2}(A, B):=h(B) .
$$

The evolution flow of the quasi-Hamiltonian system $\left(D, G, \omega, \mu, h_{1}\right)$ through the initial value $\left(A_{0}, B_{0}\right)$ is furnished by

$$
(A(t), B(t))=\left(A_{0}, B_{0} e^{-t \nabla h\left(A_{0}\right)}\right),
$$

while the system $\left(D, G, \omega, \mu, h_{2}\right)$ has the flow

$$
(A(t), B(t))=\left(A_{0} e^{t \nabla h\left(B_{0}\right)}, B_{0}\right)
$$

Indeed, the evolution vector field given by the $t$-derivative of the flow (2.15) at the point $(A(t), B(t))$ of the double equals $\left(0 \oplus-B(t) \nabla h\left(A_{0}\right)\right)$ and one can easily verify that it satisfies the defining relations (2.4) of the quasi-Hamiltonian vector field belonging to the function $h_{1}$.

In order to specify the Hamiltonians of two commuting $(n-1)$-families of quasi-Hamiltonian dynamical systems on the double, we have to introduce the so-called spectral functions on the group $G$.

As a preparation, we define the alcove $\mathcal{A}$ by

$$
\mathcal{A}:=\left\{\left(\xi_{1}, \ldots, \xi_{n}\right) \in \mathbb{R}^{n} \mid \xi_{j} \geq 0, \quad j=1, \ldots, n, \quad \sum_{j=1}^{n} \xi_{j}=\pi\right\},
$$

and the open alcove $\mathcal{A}^{0}$ by

$$
\mathcal{A}^{0}:=\left\{\left(\xi_{1}, \ldots, \xi_{n}\right) \in \mathbb{R}^{n} \mid \xi_{j}>0, \quad j=1, \ldots, n, \quad \sum_{j=1}^{n} \xi_{j}=\pi\right\} .
$$

We then consider the injective map $\delta$ from $\mathcal{A}$ into the subgroup $S \mathbb{T}_{n}$ of the diagonal elements of $S U(n)$ given by

$$
\delta_{11}(\xi):=e^{\frac{2 \mathrm{i}}{n} \sum_{j=1}^{n} j \xi_{j}}, \quad \delta_{k k}(\xi):=e^{2 \mathrm{i} \sum_{j=1}^{k-1} \xi_{j}} \delta_{11}(\xi), \quad k=2, \ldots, n .
$$


The image of $\delta$ is a fundamental domain for the action of the Weyl group of $S U(n)$ (i.e. the permutation group) on $S \mathbb{T}_{n}$, which is often called a Weyl alcove. For this reason, we may also refer to $\mathcal{A}$ as a Weyl alcove. With the aid of the fundamental weights $\lambda_{k}$ of $s u(n)$ represented by the diagonal matrices $\lambda_{k} \equiv \sum_{j=1}^{k} E_{j, j}-\frac{k}{n} \mathbf{1}_{n}$, the matrix $\delta(\xi)$ can be recast in the form

$$
\delta(\xi)=\exp \left(-2 \mathrm{i} \sum_{k=1}^{n-1} \xi_{k} \lambda_{k}\right)
$$

Here we denoted by $E_{j, j}$ the $n \times n$-matrix featuring 1 in the intersection of the $j^{\text {th }}$-row with the $j^{\text {th }}$-column and 0 everywhere else.

Every element $A \in S U(n)$ can be written as

$$
A=g(A)^{-1} \delta(\xi) g(A)
$$

for some $g(A) \in S U(n)$ and unique $\xi \in \mathcal{A}$. Moreover, whenever $\xi \in \mathcal{A}^{0}$, the element $A$ is regular and $g(A)$ is then determined up to left-multiplication by an element of $S \mathbb{T}_{n}$. By definition, the $j^{\text {th }}$ component of the alcove element $\xi$ entering the decomposition (2.21) is the value of the spectral function $\Xi_{j}$ on $A \in S U(n)$. In other words, the conjugation invariant function $\Xi_{j}$ on $G=S U(n)$ is characterized by the equation

$$
\Xi_{j}(\delta(\xi))=\xi_{j}, \quad \forall \xi \in \mathcal{A}, \quad j=1, \ldots, n .
$$

It is easily seen that the spectral function $\Xi_{j}$ is smooth on $G_{\mathrm{reg}} \subset G$, but it develops singularities at the non-regular points of $G$. Note also that $\Xi_{n}=\pi-\sum_{j=1}^{n-1} \Xi_{j}$ according to (2.17).

We are now in the position to define the $2(n-1)$ distinguished $G$-invariant Hamiltonians $\alpha_{j}, \beta_{j}$ on the double $D$ as follows:

$$
\alpha_{j}(A, B):=\Xi_{j}(A), \quad \beta_{j}(A, B):=\Xi_{j}(B), \quad j=1, \ldots, n-1 .
$$

We call $\alpha_{j}$ and $\beta_{j}$ 'spectral Hamiltonians' and our next task is to show that they respectively define commuting $(n-1)$-families of quasi-Hamiltonian dynamical systems. To be more precise, it must be noted that the domain of the $\alpha_{j}$-Hamiltonians (resp. $\beta_{j}$-Hamiltonians) is the dense open subset $D_{a} \subset D$ (resp. $D_{b} \subset D$ ) consisting of the couples $(A, B) \in D$ with $A \in G_{\text {reg }}$ (resp. $B \in G_{\mathrm{reg}}$ ), which is stable under the corresponding flows. In order to describe the flows, we now prove the following lemma.

Lemma 1. The derivative of the spectral function $\Xi_{j} \in C^{\infty}\left(G_{\mathrm{reg}}\right)(j=1, \ldots, n-1)$ reads

$$
\nabla \Xi_{j}(A)=g(A)^{-1}\left(\mathrm{i}\left(E_{j+1, j+1}-E_{j, j}\right)\right) g(A), \quad \forall A \in G_{\mathrm{reg}} .
$$

Proof. The $G$-invariance of $\Xi_{j}$ implies the $G$-equivariance of $\nabla \Xi_{j}$, and therefore it is enough to calculate $\nabla \Xi_{j}$ at the points of the open Weyl alcove. But at the points of the Weyl alcove $\nabla \Xi_{j}$ must be a diagonal matrix, because of invariance under $S \mathbb{T}_{n}$ inherited from the $G$-equivariance on $G_{\text {reg. }}$. Then $\left(\nabla \Xi_{j}\right)(\delta(\xi))$ is readily calculated to be $\mathrm{i}\left(E_{j+1, j+1}-E_{j, j}\right)$, which implies (2.24) on account of (2.21). 
Note that $\nabla \Xi_{j}(A)$ is well-defined by formula (2.24) since $g(A)$ is determined up to leftmultiplication by the elements of the maximal torus, and its smoothness on $G_{\text {reg }}$ follows directly from the smoothness of $\Xi_{j}$.

By combining the formulae (2.15), (2.16) and (2.24), we find that the following $2 \pi$-periodic curve in $D_{a}$ passing through $(A, B)$ is the integral curve of the quasi-Hamiltonian vector field $v_{\alpha_{j}}$ :

$$
\left(A, B g(A)^{-1} \operatorname{diag}\left(1,1, \ldots, 1, e^{\mathrm{i} t}, e^{-\mathrm{i} t}, 1, \ldots, 1\right) g(A)\right), \quad t \in \mathbb{R},
$$

and the following $2 \pi$-periodic curve in $D_{b}$ is an integral curve of the vector field $v_{\beta_{j}}$ :

$$
\left(A g(B)^{-1} \operatorname{diag}\left(1,1, \ldots, 1, e^{-\mathrm{i} t}, e^{\mathrm{i} t}, 1, \ldots, 1\right) g(B), B\right), \quad t \in \mathbb{R} .
$$

In particular, the formulae (2.25) and (2.26) trivially imply that the $\alpha$-flows commute among themselves and so do the $\beta$-flows. In other words, the infinitesimal actions of the commuting quasi-Hamiltonian vector fields $v_{\alpha_{j}}$ integrate to a (smooth free) action of the torus

$$
\mathbb{T}_{n-1}:=U(1)^{(n-1)}
$$

on $D_{a} \subset D$. The formula (2.25) gives the action of the $j^{\text {th }} U(1)$ factor of $\mathbb{T}_{n-1}$, the phase $e^{\mathrm{i} t}$ sits in the $j^{\text {th }}$ place of the diagonal and $g(A)$ is given by the decomposition (2.21). In spite of the ambiguity in the definition of $g(A)$, the curve (2.25) is defined unambiguously. To display the action map $\Psi^{a}: \mathbb{T}_{n-1} \times D_{a} \rightarrow D_{a}$ more explicitly, we introduce

$$
\rho(\tau):=\exp \left(\mathrm{i} \sum_{j=1}^{n-1} t_{j}\left(E_{j, j}-E_{j+1, j+1}\right)\right) \quad \text { for all } \quad \tau=\left(e^{\mathrm{i} t_{1}}, \ldots, e^{\mathrm{i} t_{n-1}}\right) \in \mathbb{T}_{n-1} .
$$

Then we have

$$
\Psi_{\tau}^{a}:(A, B) \mapsto\left(A, B g(A)^{-1} \rho(\tau) g(A)\right) .
$$

Similarly, the commuting quasi-Hamiltonian vector fields $v_{\beta_{j}}$ generate a $\mathbb{T}_{n-1}$-action on the dense open subset $D_{b} \subset D$, and the corresponding action map $\Psi^{b}: \mathbb{T}_{n-1} \times D_{b} \rightarrow D_{b}$ reads

$$
\Psi_{\tau}^{b}:(A, B) \mapsto\left(A g(B)^{-1} \rho(\tau)^{-1} g(B), B\right) .
$$

We observe also that

$$
\left\{\alpha_{j}, \alpha_{l}\right\}=0=\left\{\beta_{j}, \beta_{l}\right\}
$$

where the Poisson bracket of $G$-invariant functions was defined in Eq. (2.5). Indeed, we have

$$
\left\{\alpha_{j}, \alpha_{l}\right\} \equiv \omega\left(v_{\alpha_{j}}, v_{\alpha_{l}}\right) \equiv \mathcal{L}_{v_{\alpha_{l}}} \alpha_{j}=0
$$

where the last equality holds since the $\alpha_{l}$-generated flow acts only on the $B$-component of the double (see Eq. (2.15)) leaving therefore the $\alpha_{j}$-functions invariant. The Poisson-commutativity (2.32) of the spectral Hamiltonians $\alpha_{j}$ (and that of the $\beta_{j}$ ) survives any quasi-Hamiltonian reduction, and this fact will provide one of the underpinnings of our approach to the compactified Ruijsenaars-Schneider system. 
Remark 1. The spectral Hamiltonians $\alpha_{j}, \beta_{j}$ can be viewed as the respective generators of the Poisson-commutative rings $\mathcal{C}_{a}$ and $\mathcal{C}_{b}$ consisting of smooth invariant functions defined with the help of Eq. (2.14):

$$
\mathcal{C}_{a}:=\left\{h_{1} \in C^{\infty}\left(D_{a}\right)^{G} \mid h \in C^{\infty}\left(G_{\mathrm{reg}}\right)^{G}\right\}, \quad \mathcal{C}_{b}:=\left\{h_{2} \in C^{\infty}\left(D_{b}\right)^{G} \mid h \in C^{\infty}\left(G_{\mathrm{reg}}\right)^{G}\right\} .
$$

The rings $\mathcal{C}_{a}$ and $\mathcal{C}_{b}$ can be of course generated also by other generators, e.g. by the invariants $H_{m}(A, B) \equiv \Re \operatorname{tr}\left(A^{m}\right), H_{-m}(A, B) \equiv \Im \operatorname{tr}\left(A^{m}\right)$ and, respectively, by $F_{m}(A, B) \equiv \Re \operatorname{tr}\left(B^{m}\right)$, $F_{-m}(A, B) \equiv \Im \operatorname{tr}\left(B^{m}\right)$ for $m \in \mathbb{N}$. Although the generators $H_{ \pm m}, F_{ \pm m}$ have the apparent advantage of being globally smooth on $G$, it is more suited for our purpose to use the generators $\alpha_{j}$ and $\beta_{j}$ since their flows are $2 \pi$-periodic (this circumstance will be crucial for our arguments in Subsections 3.3 and 3.4). It will be shown that after our quasi-Hamiltonian reduction the matrix $A$ yields the Lax matrix of the Ruijsenaars-Schneider system, the generators $H_{ \pm m}$ become the Ruijsenaars-Schneider Hamiltonians, the $\alpha_{j}$ become the action-variables and the $\beta_{j}$ will parametrize the particle-positions. We shall also establish a dual interpretation of the reduction, where $B$ yields the Lax matrix, the generators $F_{ \pm m}$ become the Hamiltonians, the $\beta_{j}$ become the action-variables and the $\alpha_{j}$ the parameters of the particle-positions.

Remark 2. We note that from the viewpoint of the corresponding moduli spaces of flat connections the flows (2.15) and (2.16) are special cases of the Goldman flows [12]. The fact that the spectral functions are not smooth at the non-regular points of $G$ will cause no problem, since we shall consider a quasi-Hamiltonian reduction for which the constraint surface $\mu^{-1}\left(\mu_{0}\right)$ turns out to be a submanifold of $G_{\text {reg }} \times G_{\text {reg }} \subset D$.

\section{$3 \quad$ Reduction of the internally fused double of $S U(n)$}

As we already know, the starting point of the reduction is the choice of an element $\mu_{0} \in G$, and the corresponding constraint surface $\mu^{-1}\left(\mu_{0}\right)$ is the space of those $(A, B) \in D$ that solve the moment map constraint 4

$$
A B A^{-1} B^{-1}=\mu_{0}
$$

The simplest non-trivial possibility is to take $\mu_{0}$ from a conjugacy class of minimal but non-zero dimension. As seen from simple counting, in this case we may hope to obtain a non-trivial reduced system of dimension $2(n-1)$. Obviously, different choices from the same conjugacy class yield equivalent reduced systems. We here choose $\mu_{0}$ diagonal of the form

$$
\mu_{0}=\operatorname{diag}\left(e^{2 \mathrm{i} y}, \ldots, e^{2 \mathrm{i} y}, e^{2(1-n) \mathrm{i} y}\right), \quad y \in \mathbb{R} .
$$

Anticipating its eventual identification with the parameter of the Hamiltonian (1.1), in the next subsection we restrict $y$ to the range $0<|y|<\frac{\pi}{n}$ and then prove that $\mu_{0}$ (3.2) leads to a smooth, compact reduced phase space $P=\mu^{-1}\left(\mu_{0}\right) / G_{0}$.

In the end, we shall identify the reduced phase space with the complex projective space $\mathbb{C} P(n-1)$ and shall also obtain a full characterization of the reduced spectral Hamiltonians $\hat{\alpha}_{j}$ and $\hat{\beta}_{j}$ in terms of the standard parametrization of $\mathbb{C} P(n-1)$.

\footnotetext{
${ }^{4}$ A similar constraint equation was studied previously in a different local context [14, 10] and in complex holomorphic settings [11, 26].
} 


\subsection{The reduced phase space is smooth and compact}

Theorem 1. Consider the diagonal matrix $\mu_{0}=\operatorname{diag}\left(e^{2 \mathrm{i} y}, \ldots, e^{2 \mathrm{i} y}, e^{2(1-n) \mathrm{i} y}\right) \in S U(n)$ with a real parameter $y$ verifying

$$
0<|y|<\frac{\pi}{n}
$$

Any such $\mu_{0}$ is a strongly regular value of the moment map $\mu$ (2.12), and the corresponding reduced phase space $P=\mu^{-1}\left(\mu_{0}\right) / G_{0}$ is a smooth, compact manifold of dimension $2(n-1)$.

Proof. We first remark that $\mu^{-1}\left(\mu_{0}\right)$ is non-empty since every element of any connected, compact semi-simple Lie group can be written as a commutator [15].

To continue, note that the action (2.10) of $G$ on the double naturally descends to an action of the factor group $\bar{G}:=G / \mathbb{Z}_{n}$, where $\mathbb{Z}_{n}$ is the center of $G=S U(n)$. Similarly, the action of the adjoint isotropy group $G_{0} \subset G$ of $\mu_{0}$ on the constraint surface $\mu^{-1}\left(\mu_{0}\right)$ descends to an action of the factor group

$$
\bar{G}_{0}:=G_{0} / \mathbb{Z}_{n}
$$

It is sufficient to prove that this latter action is free. Indeed, the free action of $\bar{G}_{0}$ implies the embedded nature of $\mu^{-1}\left(\mu_{0}\right)$ by statement 3 of Proposition 4.1 of [1] (which shows that the locally free nature of the action of the isotropy group on the constraint surface is equivalent to the regularity of the moment map value). The fact that the compact Lie group $\bar{G}_{0}$ acts freely on the smooth compact manifold $\mu^{-1}\left(\mu_{0}\right)$ then ensures that

$$
\mu^{-1}\left(\mu_{0}\right) / G_{0}=\mu^{-1}\left(\mu_{0}\right) / \bar{G}_{0}
$$

also becomes a smooth compact manifold. As for its dimension, we have $\operatorname{dim}\left(\bar{G}_{0}\right)=(n-1)^{2}$, since

$$
G_{0}=S(U(n-1) \times U(1)),
$$

and therefore

$$
\operatorname{dim}\left(\mu^{-1}\left(\mu_{0}\right) / \bar{G}_{0}\right)=(n-1)(n+1)-(n-1)^{2}=2(n-1) .
$$

It remains to prove that if $(A, B) \in \mu^{-1}\left(\mu_{0}\right)$ is fixed by some $g \in S U(n)$, then $g$ belongs to the central subgroup $\mathbb{Z}_{n}$. For this, suppose that $\left(g A g^{-1}, g B g^{-1}\right)=(A, B)$ holds for some $(A, B) \in \mu^{-1}\left(\mu_{0}\right)$ and $g \in G$. This implies that both $A$ and $B$ belong to the centralizer subgroup

$$
G(g):=\left\{\eta \in S U(n) \mid \eta g \eta^{-1}=g\right\} \subseteq S U(n),
$$

and $\mu_{0}=A B A^{-1} B^{-1}$ belongs to the corresponding derivative subgroup $G(g)^{\prime}$ that contains the group-commutators in $G(g)$. Now observe that if $g$ is not central, then it is conjugate to an element $g_{0}$ of the maximal torus of $S U(n)$ whose centralizer $G\left(g_{0}\right)$ is a block-diagonal subgroup

$$
G\left(g_{0}\right)=S\left(U\left(n_{1}\right) \times U\left(n_{2}\right) \times \cdots \times U\left(n_{k}\right)\right),
$$

for some $k \geq 2$ and positive integers for which $n_{1}+n_{2}+\cdots+n_{k}=n\left(k=1\right.$ occurs for $\left.g \in \mathbb{Z}_{n}\right)$. Accordingly, if $g$ is not central, then $\mu_{0}$ must be conjugate to an element of the commutator subgroup $G\left(g_{0}\right)^{\prime}$ of $G\left(g_{0}\right)$ (3.9). It is readily seen that $G\left(g_{0}\right)^{\prime}$ is provided by

$$
G\left(g_{0}\right)^{\prime}=S U\left(n_{1}\right) \times S U\left(n_{2}\right) \times \cdots \times S U\left(n_{k}\right),
$$


which leads to a contradiction. Indeed, it follows from (3.3) that in whatever way we partition the $n$ eigenvalues of $\mu_{0}$ into $k>1$ parts, the product of the eigenvalues in at least one part (actually in each part) will not be equal to 1 . Thus $\mu_{0}$ cannot be conjugate to an element of $G\left(g_{0}\right)^{\prime}(3.10)$ for $k>1$.

We remark in passing that the above arguments show also the strong regularity of any such moment map value from $S U(n)$ which is not conjugate to a block-diagonal $S U(n)$ matrix whose blocks themselves have determinant 1.

\subsection{The images of the Hamiltonians $\alpha_{j}, \beta_{j}$ restricted to $\mu^{-1}\left(\mu_{0}\right)$}

Having established that the reduced phase space $\mu^{-1}\left(\mu_{0}\right) / G_{0}$ is a compact smooth manifold, the next step is to determine the reduced symplectic form $\hat{\omega}$ on it. Remarkably, the shortest way to this goal leads through the study of the images of the spectral Hamiltonians $\alpha_{j}, \beta_{j}$ (2.23) restricted to the constraint surface $\mu^{-1}\left(\mu_{0}\right)$.

Theorem 2. For $\mu_{0}=\operatorname{diag}\left(e^{2 \mathrm{i} y}, \ldots, e^{2 \mathrm{i} y}, e^{2(1-n) \mathrm{i} y}\right)$ with $0<|y|<\frac{\pi}{n}$, the convex polytope

$$
\mathcal{P}_{y}:=\left\{\left(\xi_{1}, \ldots, \xi_{n-1}\right) \in \mathbb{R}^{n-1}\left|\xi_{j} \geq\right| y\left|, \quad j=1, \ldots, n-1, \sum_{j=1}^{n-1} \xi_{j} \leq \pi-\right| y \mid\right\}
$$

is the common image of the vector-valued Hamiltonian functions $\left(\alpha_{1}, \ldots, \alpha_{n-1}\right)$ and $\left(\beta_{1}, \ldots, \beta_{n-1}\right)$ restricted to the constraint surface $\mu^{-1}\left(\mu_{0}\right)$.

Proof. The formulation of Theorem 2 in terms of the convex polytope $\mathcal{P}_{y}$ will play an important role in Section 3.4. However, from the technical point of view, it is more convenient to include into the analysis also the functions $\alpha_{n}(A, B):=\Xi_{n}(A), \beta_{n}(A, B):=\Xi_{n}(B)$ (cf. (2.22) $-(2.23)$ ) and to prove the following equivalent statement:

The common image of the vector-valued functions $\left(\alpha_{1}, \ldots, \alpha_{n-1}, \alpha_{n}\right)$ and $\left(\beta_{1}, \ldots, \beta_{n-1}, \beta_{n}\right)$ restricted to the constraint surface $\mu^{-1}\left(\mu_{0}\right)$ is the set

$$
\mathcal{A}_{y}:=\left\{\left(\xi_{1}, \ldots, \xi_{n}\right) \in \mathbb{R}^{n}\left|\xi_{j} \geq\right| y \mid, \quad j=1, \ldots, n, \sum_{j=1}^{n} \xi_{j}=\pi\right\} .
$$

The constraint $A B A^{-1} B^{-1}=\operatorname{diag}\left(e^{2 \mathrm{i} y}, \ldots, e^{2 \mathrm{i} y}, e^{2(1-n) \mathrm{i} y}\right)$ is invariant under the interchange of $A$ and $B$ accompanied with a simultaneous change of the sign of the parameter $y$. Since $\mathcal{A}_{y}$ does not depend on the sign of $y$, it is enough to show that the image of $\left(\beta_{1}, \ldots, \beta_{n-1}, \beta_{n}\right)$ restricted to $\mu^{-1}\left(\mu_{0}\right)$ is $\mathcal{A}_{y}$.

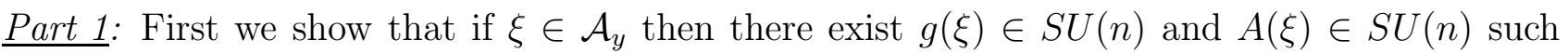
that $A(\xi)$ and $B(\xi):=g(\xi)^{-1} \delta(\xi) g(\xi)$ solve the moment map constraint (3.1). (Recall that the map $\delta: \mathcal{A} \rightarrow S \mathbb{T}_{n}$ was defined in Eq. (2.19) in connection with the decomposition (2.21); below we use $\delta_{j}:=\delta_{j j}$.)

Consider an arbitrary $\xi=\left(\xi_{1}, \ldots, \xi_{n}\right) \in \mathcal{A}_{y}$ and define

$$
\xi_{k n+j}:=\xi_{j}, \quad k \in \mathbb{Z}, \quad j=1, \ldots, n .
$$


As an immediate consequence of Eq. (2.19), note the validity of the following relation:

$$
\delta_{j}(\xi) \delta_{l}(\xi)^{-1}=\exp \left(2 \mathrm{i} \sum_{k=l}^{j-1} \xi_{k}\right), \quad 1 \leq l<j \leq n .
$$

Moreover, using the convention (3.13), we have

$$
\cot |y| \geq\left|\cot \left(\sum_{k=l}^{j-1} \xi_{k}\right)\right|, \quad l=1, \ldots, n ; \quad j=l+1, \ldots, l+n-1,
$$

because $\sum_{k=l}^{j-1} \xi_{k}$ in (3.15) always lies in the closed interval $[|y|, \pi-|y|]$. Thus for $\xi \in \mathcal{A}_{y}$ and $l=1, \ldots, n$ we obtain the reality and non-negativity of the quantities $z_{l}(\delta(\xi), y)$ defined by

$$
z_{l}(\delta(\xi), y):=\frac{e^{2 \mathrm{i} y}-1}{e^{2 n \mathrm{i} y}-1} \prod_{j \neq l}^{n} \frac{\delta_{j}(\xi)-e^{2 \mathrm{i} y} \delta_{l}(\xi)}{\delta_{j}(\xi)-\delta_{l}(\xi)}=\frac{(\sin |y|)^{n}}{\sin (n|y|)} \prod_{j=l+1}^{l+n-1}\left(\cot |y|-\frac{y}{|y|} \cot \left(\sum_{k=l}^{j-1} \xi_{k}\right)\right)
$$

Note that the second equality in (3.16) follows from (3.14) and from the following trigonometric identity:

$$
\cot y-\cot \beta \equiv \frac{\sin (\beta-y)}{\sin y \sin \beta} \equiv 2 \mathrm{i} \frac{e^{2 \mathrm{i} \beta}-e^{2 \mathrm{i} y}}{\left(e^{2 \mathrm{i} y}-1\right)\left(e^{2 \mathrm{i} \beta}-1\right)} .
$$

Now consider an arbitrary map $v: \mathcal{A}_{y} \rightarrow \mathbb{C}^{n}$ such that

$$
\left|v_{l}(\xi)\right|^{2}:=z_{l}(\delta(\xi), y)
$$

Let us show then that

$$
\|v(\xi)\|^{2}:=\sum_{l=1}^{n}\left|v_{l}(\xi)\right|^{2}=1
$$

For this, we first check the equality of the following two polynomials in an auxiliary complex variable $\lambda$ :

$$
\prod_{j=1}^{n}\left(\delta_{j}(\xi)-\lambda\right)=\prod_{j=1}^{n}\left(\delta_{j}(\xi) \epsilon^{2 \mathrm{i} y}-\lambda\right)+\left(e^{2 \mathrm{i}(1-n) y}-e^{2 \mathrm{i} y}\right) \sum_{k=1}^{n}\left(\left|v_{k}(\xi)\right|^{2} \delta_{k}(\xi) \prod_{j \neq k}^{n}\left(\delta_{j}(\xi) e^{2 \mathrm{i} y}-\lambda\right)\right) .
$$

Indeed, it is easy to verify (3.20) for the $n$ (all distinct) values $\lambda_{j}=\delta_{j}(\xi) e^{2 \mathrm{i} y}, j=1, \ldots, n$. Consequently, (3.20) holds true for any $\lambda$, and we obtain (3.19) by evaluating (3.20) for $\lambda=0$.

We note that the polynomial identity (3.20) can be understood as the equality of the characteristic polynomials of the diagonal matrix $\delta(\xi)$ and of the matrix $\mu_{v(\xi)} \delta(\xi)$,

$$
\operatorname{det}\left(\delta(\xi)-\lambda \mathbf{1}_{n}\right)=\operatorname{det}\left(\mu_{v(\xi)} \delta(\xi)-\lambda \mathbf{1}_{n}\right)
$$

where the matrix $\mu_{v(\xi)}$ reads

$$
\mu_{v(\xi)}:=e^{2 \mathrm{i} y} \mathbf{1}_{n}+\left(e^{2 \mathrm{i}(1-n) y}-e^{2 \mathrm{i} y}\right) v(\xi) v(\xi)^{\dagger} .
$$


Because of the normalization property (3.19), there certainly exists an $S U(n)$ matrix $g(\xi)$ having the vector $v(\xi)$ as its last column, i.e., $v_{j}(\xi)=g(\xi)_{j n}$. It is then easily seen that the diagonal moment map value $\mu_{0}(3.2)$ can be written as

$$
\mu_{0}=g(\xi)^{-1} \mu_{v(\xi)} g(\xi)
$$

and the determinant identity (3.21) can be therefore rewritten as

$$
\operatorname{det}\left(g(\xi)^{-1} \delta(\xi) g(\xi)-\lambda \mathbf{1}_{n}\right)=\operatorname{det}\left(\mu_{0} g(\xi)^{-1} \delta(\xi) g(\xi)-\lambda \mathbf{1}_{n}\right) .
$$

This means that the matrix $B(\xi):=g(\xi)^{-1} \delta(\xi) g(\xi)$ has the same spectrum as the matrix $\mu_{0} B(\xi)$, which implies the existence of a matrix $A(\xi) \in S U(n)$ such that

$$
A(\xi) B(\xi) A(\xi)^{-1}=\mu_{0} B(\xi) .
$$

Part 1 of the proof of Theorem 2 is thus complete.

Part 2: It remains to show that if $(A, B) \in D$ satisfies the moment map constraint (3.1), then $B$ can be written as

$$
B=g^{-1} \delta(\xi) g
$$

with some $g \in S U(n)$ and some $\xi \in \mathcal{A}_{y}$ (3.12). Using that any $B \in S U(n)$ has the form (3.26) with uniquely determined $\xi \in \mathcal{A}(2.17)$, it will be convenient to distinguish two cases: i) $\xi$ is in the open Weyl alcove $\mathcal{A}^{0}(2.18)$; ii) $\xi \notin \mathcal{A}^{0}$. We consider first i) and then ii). More precisely, we shall first prove the statement:

i) If $(A, B) \in \mu^{-1}\left(\mu_{0}\right), B=g^{-1} \delta(\xi) g$ for some $g \in S U(n)$ and $\xi \in \mathcal{A}^{0}$, then $\xi \in \mathcal{A}_{y}$.

Then we prove the statement:

ii) If $\xi \notin \mathcal{A}^{0}$ then whatever is $g \in S U(n)$, the matrix $B=g^{-1} \delta(\xi) g$ cannot be the second component of some solution $(A, B)$ of the constraint (3.1).

Proof of statement $i)$ : Define $A_{g}$ and $\mu_{g}$ as

$$
A_{g}:=g A g^{-1}, \quad \mu_{g}:=g \mu_{0} g^{-1} .
$$

The validity of (3.1) implies

$$
A_{g} \delta(\xi) A_{g}^{-1}=\mu_{g} \delta(\xi)
$$

Note that the matrix $\mu_{g}$ depends only on the last column of the matrix $g$. To see this we rewrite $\mu_{0}$ as

$$
\mu_{0}=e^{2 \mathrm{i} y} \mathbf{1}_{n}+\left(e^{2 \mathrm{i}(1-n) y}-e^{2 \mathrm{i} y}\right) v_{0} v_{0}^{\dagger},
$$

where the vector $v_{0} \in \mathbb{C}^{n}$ is defined by its components $\left(v_{0}\right)_{n}:=1,\left(v_{0}\right)_{j}:=0, j=1, \ldots, n-1$. This means that $\mu_{g}$ can be written as

$$
\mu_{g}=e^{2 \mathrm{i} y} \mathbf{1}_{n}+\left(e^{2 \mathrm{i}(1-n) y}-e^{2 \mathrm{i} y}\right)\left(g v_{0}\right)\left(g v_{0}\right)^{\dagger}=e^{2 \mathrm{i} y} \mathbf{1}_{n}+\left(e^{2 \mathrm{i}(1-n) y}-e^{2 \mathrm{i} y}\right) v v^{\dagger},
$$

where $v:=g v_{0}$ is the last column of the matrix $g$, i.e., $v_{j}=g_{j n}$.

We observe from (3.28) that the spectrum of the matrix $\mu_{g} \delta(\xi)$ must be equal to the spectrum of $\delta(\xi)$, which entails the equality of the characteristic polynomials

$$
\operatorname{det}\left(\delta(\xi)-\lambda \mathbf{1}_{n}\right)=\operatorname{det}\left(\mu_{g} \delta(\xi)-\lambda \mathbf{1}_{n}\right) .
$$


Both determinants in (3.31) can be easily evaluated so that (3.31) becomes

$$
\prod_{j=1}^{n}\left(\delta_{j}(\xi)-\lambda\right)=\prod_{j=1}^{n}\left(\delta_{j}(\xi) \epsilon^{2 \mathrm{i} y}-\lambda\right)+\left(e^{2 \mathrm{i}(1-n) y}-e^{2 \mathrm{i} y}\right) \sum_{k=1}^{n}\left(\left|v_{k}\right|^{2} \delta_{k}(\xi) \prod_{j \neq k}^{n}\left(\delta_{j}(\xi) e^{2 \mathrm{i} y}-\lambda\right)\right) .
$$

Due to the assumption $\xi \in \mathcal{A}^{0}$, we know that the elements of the diagonal matrix $\delta(\xi)$ have $n$ distinct values. By evaluating the relation (3.32) for the $n$ distinct values $\lambda_{l}=\delta_{l}(\xi) e^{2 \mathrm{i} y}$, we immediately find

$$
\left|v_{l}\right|^{2}=\frac{e^{2 \mathrm{i} y}-1}{e^{2 n \mathrm{i} y}-1} \prod_{j \neq l}^{n} \frac{\delta_{j}(\xi)-e^{2 \mathrm{i} y} \delta_{l}(\xi)}{\delta_{j}(\xi)-\delta_{l}(\xi)}=\frac{(\sin |y|)^{n}}{\sin (n|y|)} \prod_{j=l+1}^{l+n-1}\left(\cot |y|-\frac{y}{|y|} \cot \left(\sum_{k=l}^{j-1} \xi_{k}\right)\right) .
$$

Now we have to distinguish whether $y>0$ or $y<0$. We start with $y>0$. Then the first term in the last product in (3.33) is $\cot y-\cot \xi_{l}$. If $\xi_{l}$ was strictly inferior to $|y|$ for a certain $l$, we would have obviously

$$
\cot |y|-\cot \xi_{l}<0
$$

If we then had $\cot |y|-\cot \left(\xi_{l}+\xi_{l+1}\right)>0$, this would imply $\cot |y|-\cot \left(\xi_{l}+\xi_{l+1}+\xi_{l+2}\right)>0$ etc, which would give $\left|v_{l}\right|^{2}<0$ by (3.33). In order to avoid such a contradiction, we see that the assumption $\xi_{l}<|y|$ leads to

$$
\cot |y|-\cot \left(\xi_{l}+\xi_{l+1}\right) \leq 0
$$

and hence to

$$
\xi_{l}+\xi_{l+1} \leq|y|
$$

Because $\xi \in \mathcal{A}^{0}$, we have $\xi_{l}>0$ and $\xi_{l+1}>0$. This fact together with (3.36) gives

$$
\xi_{l+1}<|y|
$$

Thus, we have shown that if $\xi_{l}<|y|$ for some $l$ then $\xi_{l+1}<|y|$, and hence $\xi_{j}<|y|$ for all $j=1, \ldots, n$. This is a contradiction since we know, respectively, from (2.17) and (3.2) that $\sum_{j=1}^{n} \xi_{j}=\pi$ and $n|y|<\pi$ hold. We conclude that $\xi_{l} \geq|y|$ for all $l=1, \ldots, n$, whereby statement i) is proved for $y>0$.

If $y<0$, note that the last term in the last product in (3.33) is equal to $\cot |y|-\cot \xi_{l-1}$. If $\xi_{l-1}$ was strictly inferior to $|y|$, we would have obviously

$$
\cot |y|-\cot \xi_{l-1}<0 \text {. }
$$

If moreover the next to last term, $\cot |y|-\cot \left(\xi_{l-1}+\xi_{l-2}\right)$, was strictly positive, this would give $\left|v_{l}\right|^{2}<0$ because all preceding terms would have to be strictly positive, too. Thus, the assumption $\xi_{l-1}<|y|$ leads to $\cot |y|-\cot \left(\xi_{l-1}+\xi_{l-2}\right) \leq 0$. This implies $\xi_{l-2}<|y|$, and consequently $\xi_{j}<|y|$ for all $j$. But this creates the same contradiction as in the case $y>0$, whereby the proof of statement i) is complete.

Proof of statement ii): To start, we note that the condition that $\xi \in \mathcal{A}$ but $\xi \notin \mathcal{A}^{0}(2.18)$ means that there exists at least one index $l \in\{1, \ldots, n\}$ for which $\xi_{l}=0$. We call such a configuration 
$\xi$ degenerate, since it is characterized by the fact that the phases $\delta_{j}(\xi)$ take only $r<n$ distinct values. We find it more convenient to describe the degenerate configurations directly by their phases $\delta_{j}(\xi)$. Since the map $\delta: \mathcal{A} \rightarrow S \mathbb{T}_{n}$ is injective, such a description is equivalent to the previous description in terms $\xi$ and from now on we simply write $\delta_{j}$ instead of $\delta_{j}(\xi)$.

Fixing an arbitrary degenerate configuration, we partition $n$ as a sum of $1 \leq r<n$ positive integers,

$$
n=k_{1}+k_{2}+\ldots+k_{r}
$$

in such a way that

$$
\delta_{1}=\delta_{2}=\ldots=\delta_{k_{1}}, \quad \delta_{k_{1}+1}=\delta_{k_{1}+2}=\ldots=\delta_{k_{1}+k_{2}}, \ldots, \delta_{\sum_{i=1}^{r-1} k_{i}+1}=\delta_{\sum_{i=1}^{r-1} k_{i}+2}=\ldots=\delta_{n} .
$$

Plainly, at least one integer $k_{s}(1 \leq s \leq r)$ must be superior or equal to 2 .

Define the matrices $A_{g}, \mu_{g}$ and the vector $v$ in the same way as in the proof of statement i). Then the assumed validity of the relation $A_{g} \delta A_{g}^{-1}=\mu_{g} \delta$ entails the equality of the characteristic polynomials of the matrices $\delta$ and $\mu_{g} \delta$, which now yields

$$
\prod_{j=1}^{r}\left(\Delta_{j}-\lambda\right)^{k_{j}}=\prod_{j=1}^{r}\left(\Delta_{j} e^{2 \mathrm{i} y}-\lambda\right)^{k_{j}}+\left(e^{2 \mathrm{i}(1-n) y}-e^{2 \mathrm{i} y}\right) \sum_{m=1}^{r} Z_{m} \Delta_{m}\left(\Delta_{m} e^{2 \mathrm{i} y}-\lambda\right)^{k_{m}-1} \prod_{j \neq m}^{r}\left(\Delta_{j} e^{2 \mathrm{i} y}-\lambda\right)^{k_{j}} .
$$

Here we introduced $r$ distinct variables $\Delta_{s}(s=1, \ldots, r)$,

$$
\Delta_{1}:=\delta_{k_{1}}, \quad \Delta_{2}:=\delta_{k_{1}+k_{2}}, \ldots, \Delta_{r}:=\delta_{k_{1}+k_{2}+\ldots+k_{r}} \equiv \delta_{n}
$$

and $r$ non-negative real variables $Z_{s}$,

$$
Z_{1}:=\left|v_{1}\right|^{2}+\left|v_{2}\right|^{2}+\ldots+\left|v_{k_{1}}\right|^{2}, \ldots, Z_{j+1}:=\left|v_{k_{1}+\ldots+k_{j}+1}\right|^{2}+\left|v_{k_{1}+\ldots+k_{j}+2}\right|^{2}+\ldots+\left|v_{k_{1}+k_{2}+\ldots+k_{j+1}}\right|^{2}
$$

for all $j=1, \ldots, r-1$. Due to the degeneracy of $\delta$, the implications of (3.41) are qualitatively different from the implications of its relative (3.32) obtained in case i). To see this, we now rewrite equation (3.41) as a relation between two rational functions of $\lambda$ :

$Q(\Delta, y, \lambda):=\prod_{j=1}^{r} \frac{\left(\Delta_{j}-\lambda\right)^{k_{j}}}{\left(\Delta_{j} e^{2 \mathrm{i} y}-\lambda\right)^{k_{j}-1}}=\prod_{j=1}^{r}\left(\Delta_{j} e^{2 \mathrm{i} y}-\lambda\right)+\left(e^{2 \mathrm{i}(1-n) y}-e^{2 \mathrm{i} y}\right) \sum_{m=1}^{r} Z_{m} \Delta_{m} \prod_{j \neq m}^{r}\left(\Delta_{j} e^{2 \mathrm{i} y}-\lambda\right)$.

Eq. (3.44) says that the variables $\Delta_{s}$ (3.42) must be such that the rational function $Q(\Delta, y, \lambda)$ is a polynomial in $\lambda$. This means that all putative poles of $Q(\Delta, y, \lambda)$ must be cancelled by appropriate monomials present in the numerator. The necessary and sufficient condition for this to occur is easily seen to be the following:

For every index $m \in\{1, \ldots, r\}$ such that $k_{m}>1$, there must exist an index $s \in\{1, \ldots, r\}$ such that $\Delta_{s}=\Delta_{m} e^{2 \mathrm{i} y}$ and $k_{s} \geq k_{m}-1$.

From now on we consider only the admissible degenerate $\delta$-configurations that, by definition, satisfy the condition $(*)$. (We saw that other degenerate configurations cannot occur in the spectrum of the matrix $B$ solving the constraint (3.1).) Taking any such configuration, with $\Delta$ in (3.42), we can find the uniquely determined quantities $Z_{m}=Z_{m}(\Delta, y)(m=1, \ldots, r)$ for 
which the relation (3.44) is satisfied. For this, it is sufficient to use $r$ different values of the parameter $\lambda$ given by $\lambda_{m}=\Delta_{m} e^{2 \mathrm{i} y}$, whereby we obtain $Z_{m}(\Delta, y)$ from (3.44). However, in distinction to the non-degenerate cases, here three possibilities may occur. First, if $k_{m}=1$ and there exists no such index $s$ for which $\Delta_{s}=\Delta_{m} e^{2 \mathrm{i} y}$, then we find

$$
Z_{m}(\Delta, y)=\frac{e^{2 \mathrm{i} y}-1}{e^{2 n \mathrm{i} y}-1} \prod_{j \neq m}^{r}\left(\frac{\Delta_{j}-e^{2 \mathrm{i} y} \Delta_{m}}{\Delta_{j}-\Delta_{m}}\right)^{k_{j}} \neq 0 .
$$

Second, if $k_{m}>1$ and $k_{s}=k_{m}-1$, then

$$
Z_{m}(\Delta, y)=(-1)^{k_{m}+1} e^{2\left(k_{m}-1\right) \mathrm{i} y} \frac{e^{2 \mathrm{i} y}-1}{e^{2 n \mathrm{i} y}-1} \prod_{j \neq m, s}^{r}\left(\frac{\Delta_{j}-e^{2 \mathrm{i} y} \Delta_{m}}{\Delta_{j}-\Delta_{m}}\right)^{k_{j}} \neq 0 .
$$

Here and below, it should not cause any confusion that we suppressed the dependence of $s$ on $m$ as given by the condition $\left(^{*}\right)$. Third, in the rest of the cases, for which either $k_{m}=1$ and there exists an index $s$ with $\Delta_{s}=\Delta_{m} e^{2 \mathrm{i} y}$ or $k_{m}>1$ and $k_{s}>k_{m}-1$, we obtain

$$
Z_{m}(\Delta, y)=0
$$

Let $S(\delta)$ denote the set of the integers $m$ that occur in Eqs. (3.45) and (3.46). This set cannot be empty, since otherwise all components of the vector $v=g v_{0}$ of unit norm were zero (cf. (3.43) ). We are going to finish the proof of statement ii) by showing that Eqs. (3.45) and (3.46) imply that at least one of the a priori non-negative quantities $Z_{m}(\Delta, y)$ is necessarily strictly negative, whatever is the admissible degenerate $\delta$-configuration that we consider. To this end, we introduce a real positive parameter $\epsilon$ and associate to every admissible degenerate $\delta$-configuration a continuous $\epsilon$-family of configurations $\delta_{\epsilon}$ in the open Weyl alcove $\delta\left(\mathcal{A}^{0}\right) \subset S \mathbb{T}_{n}$ :

$$
\delta_{\epsilon, p}:=\Delta_{1} e^{\mathrm{i} p \epsilon}, \quad p=1, \ldots, k_{1} ; \quad \delta_{\epsilon, k_{1}+\ldots+k_{j-1}+p}:=\Delta_{j} e^{\mathrm{i} p \epsilon}, \quad p=1, \ldots, k_{j}, \quad j=2, \ldots, r,
$$

where we use the partition (3.39). It is evident that for sufficiently small values of $\epsilon>0$ the configurations $\delta_{\epsilon}$ are non-degenerate, i.e., they sit in the $\delta$-image of $\mathcal{A}^{0}$ (defined in (2.18) and $(2.19))$. Consider now for $l=1, \ldots, n$ the quantities $z_{l}\left(\delta_{\epsilon}, y\right)$,

$$
z_{l}\left(\delta_{\epsilon}, y\right):=\frac{e^{2 \mathrm{i} y}-1}{e^{2 n \mathrm{i} y}-1} \prod_{j \neq l}^{n} \frac{\delta_{\epsilon, j}-e^{2 \mathrm{i} y} \delta_{\epsilon, l}}{\delta_{\epsilon, j}-\delta_{\epsilon, l}}
$$

which appeared also in the formula (3.16). From the fact that the configuration is admissible $\left(^{*}\right)$ it follows easily that for some l's the quantities $z_{l}\left(\delta_{\epsilon}, y\right)$ vanish. More precisely, we first observe that

$$
z_{l}\left(\delta_{\epsilon}, y\right)=0 \quad \text { if } \quad l \notin\left\{k_{1}, k_{1}+k_{2}, \ldots, k_{1}+\ldots+k_{r}\right\}
$$

and also

$$
z_{k_{1}+k_{2}+\ldots+k_{m}}\left(\delta_{\epsilon}, y\right)=0 \quad \forall m \text { for which } \exists s \text { such that } \Delta_{s}=e^{2 \mathrm{i} y} \Delta_{m} \text { and } k_{s} \geq k_{m} .
$$

Moreover, it turns out that the limits $\lim _{\epsilon \rightarrow 0} z_{l}\left(\delta_{\epsilon}, y\right)$ exist and do not vanish for all other $l$. That is they do not vanish for all $l=k_{1}+k_{2}+\ldots+k_{m}$ for which one of the following alternatives 
occurs: a) $k_{m}=1$ and there is no $s$ such that $\Delta_{s}=e^{2 \mathrm{i} y} \Delta_{m}$; b) $k_{m}>1, \Delta_{s}=e^{2 \mathrm{i} y} \Delta_{m}$ and $k_{s}=k_{m}-1$. Those non-vanishing limits read

$$
\lim _{\epsilon \rightarrow 0} z_{k_{1}+\ldots+k_{m}}\left(\delta_{\epsilon}, y\right)=Z_{m}(\Delta, y)
$$

where $Z_{m}(\Delta, y)$ is given by Eqs. (3.45) or (3.46) for the cases a) and b), respectively. In other words, the $m$-values occurring in (3.52) form the set $S(\delta)$ defined after (3.47).

Now turning to the crux of the argument, we note that for small $\epsilon$ the configuration $\delta_{\epsilon}$ does not belong to the $\delta$-image of $\mathcal{A}_{y}(3.12)$. Indeed, whenever $k_{j}>1$ (recall that such $1 \leq j \leq r$ exists), we observe that in the $\xi$-parametrization (2.19) of the configuration $\delta_{\epsilon}$ we have $\xi_{k_{j}-1}=$ $\epsilon / 2<|y|$. Following the proof of statement i), the quantity $z_{l}\left(\delta_{\epsilon}, y\right)$ must be therefore strictly negative at least for one (in principle $\epsilon$-dependent) value of $l$. As an obvious consequence, there must also exist a fixed index $l$ and a decreasing series $\epsilon_{p} \rightarrow 0$ such that $z_{l}\left(\delta_{\epsilon_{p}}, y\right)$ is strictly negative for all positive integers $p$. From (3.50) and (3.51), we conclude the existence of an integer $m^{*} \in S(\delta)$ such that the above mentioned $\epsilon$-independent $l$ is given by $l=k_{1}+k_{2}+\ldots+k_{m^{*}}$. We know from (3.52) that the limit

$$
\lim _{p \rightarrow \infty} z_{k_{1}+\ldots+k_{m^{*}}}\left(\delta_{\epsilon_{p}}, y\right)=Z_{m^{*}}(\Delta, y)
$$

does not vanish, which implies that $Z_{m^{*}}(\Delta, y)$ must be strictly negative. This is a contradiction with the non-negativity of the variables $Z_{m}$ (3.43).

\subsection{The reduced phase space is a Hamiltonian toric manifold}

By definition, a Hamiltonian toric manifold 5 is a compact, connected symplectic manifold of dimension $2 d$ equipped with an effective, Hamiltonian action of a torus of dimension $d$. We already know that the reduced phase space $P=\mu^{-1}\left(\mu_{0}\right) / G_{0}$ is a compact symplectic manifold. The following Lemmas 2 and 3 show that $P$ is a Hamiltonian toric manifold. Lemma 2 refers to the $\beta$-generated torus action (2.30), but of course an analogous result holds also for the $\alpha$-generated action (2.29); and eventually this will explain the Ruijsenaars self-duality.

Lemma 2. The $\beta$-generated $\mathbb{T}_{n-1}$-action on the open submanifold $D_{b}$ of the internally fused double $D$, given by (2.30), descends to the reduced phase space $P=\mu^{-1}\left(\mu_{0}\right) / G_{0}$, where it becomes Hamiltonian and effective.

Proof. It follows from Theorem 2 that the constraint surface $\mu^{-1}\left(\mu_{0}\right) \subset D$ lies completely in the open submanifold $D_{b} \subset D$ (recall that $D_{b}$ is the set of pairs $(A, B) \in D$ for which $B$ is regular). Thus, the statement that the torus action $\Psi^{b}(2.30)$ descends to a Hamiltonian torus action on the reduced phase space follows immediately from the general theory of quasi-Hamiltonian reduction [1], which we briefly summarized around equation (2.7). In fact, the reduced torus action $\hat{\Psi}^{b}: \mathbb{T}_{n-1} \times P \rightarrow P$ can be defined by means of the equality

$$
\hat{\Psi}_{\tau}^{b} \circ p=p \circ\left(\left.\Psi_{\tau}^{b}\right|_{\mu^{-1}\left(\mu_{0}\right)}\right), \quad \forall \tau \in \mathbb{T}_{n-1},
$$

\footnotetext{
${ }^{5}$ The general theory of these compact completely integrable systems is reviewed, for example, in [2].
} 
where $p: \mu^{-1}\left(\mu_{0}\right) \rightarrow P$ is the canonical projection. The corresponding infinitesimal torus action on $P$ is generated by the vector fields $\hat{v}_{\hat{\beta}_{j}}(j=1, \ldots, n-1)$ that are the projections of the vector fields $v_{\beta_{j}}(2.26)$ restricted to $\mu^{-1}\left(\mu_{0}\right)$. These projected vector fields are Hamiltonian,

$$
\hat{\omega}\left(\hat{v}_{\hat{\beta}_{j}}, \cdot\right)=d \hat{\beta}_{j}
$$

where $\hat{\omega}$ is the reduced symplectic form on $P$ and the reduced Hamiltonians $\hat{\beta}_{j} \in C^{\infty}(P)$ are characterized by $\hat{\beta}_{j} \circ p=\beta_{j} \circ \iota$ using the embedding $\iota: \mu^{-1}\left(\mu_{0}\right) \rightarrow D$. In other words,

$$
\hat{\beta} \equiv\left(\hat{\beta}_{1}, \ldots, \hat{\beta}_{n-1}\right): P \rightarrow \mathbb{R}^{n-1}
$$

is the moment map for the $\mathbb{T}_{n-1}$-action $\hat{\Psi}^{b}$ on $P$.

Suppose now that the $\mathbb{T}_{n-1}$-action $\hat{\Psi}^{b}$ on $P$ is not effective. We observe from (2.30) that this is equivalent to the existence of a non-unit element $\rho \in S \mathbb{T}_{n}$ such that for all $(A, B) \in \mu^{-1}\left(\mu_{0}\right)$ there exists an element $h(A, B) \in G_{0}$ satisfying

$$
\left(A g(B)^{-1} \rho g(B), B\right)=\left(h(A, B) A h(A, B)^{-1}, h(A, B) B h(A, B)^{-1}\right) .
$$

This means that $\hat{\Psi}_{\tau}^{b}(p(A, B))=p(A, B)$ for the element $\tau \in \mathbb{T}_{n-1}$ for which $\rho=\rho(\tau)$ according to (2.28). Note from (3.57) that $h(A, B)$ must commute with $B$. Because $(A, B) \in \mu^{-1}\left(\mu_{0}\right)$, $B=g(B)^{-1} \delta(\xi) g(B)$ is regular by Theorem 2 and therefore there exists some $(A, B)$-dependent $\zeta=\operatorname{diag}\left(\zeta_{1}, \ldots, \zeta_{n}\right) \in S \mathbb{T}_{n}$ such that

$$
h(A, B)=g(B)^{-1} \zeta g(B) .
$$

The fact that $h(A, B) \in G_{0}$ then says that

$$
g(B)^{-1} \zeta g(B) \mu_{0}=\mu_{0} g(B)^{-1} \zeta g(B),
$$

or, equivalently,

$$
\zeta g(B) \mu_{0} g(B)^{-1}=g(B) \mu_{0} g(B)^{-1} \zeta .
$$

We know from the proof of Theorem 2 that the last column of the matrix $g(B)$ is given by a vector $v(\xi)$ verifying

$$
\left|v_{l}(\xi)\right|^{2}=\frac{(\sin |y|)^{n}}{\sin (n|y|)} \prod_{j=l+1}^{l+n-1}\left(\cot |y|-\frac{y}{|y|} \cot \left(\sum_{k=l}^{j-1} \xi_{k}\right)\right), \quad \forall l=1, \ldots, n,
$$

where $\xi \in \mathcal{A}_{y}(\underline{3.12})$. If $\xi$ belongs to the interior $\mathcal{A}_{y}^{0}$ of $\mathcal{A}_{y}$,

$$
\mathcal{A}_{y}^{0}:=\left\{\left(\xi_{1}, \ldots, \xi_{n}\right) \in \mathbb{R}^{n}\left|\xi_{j}>\right| y \mid, \quad j=1, \ldots, n, \quad \sum_{j=1}^{n} \xi_{j}=\pi\right\},
$$

then all components of the vector $v(\xi)$ are non-vanishing. In this case we compare the last columns of the matrices on the two sides of Eq. (3.60). By using the formula of $\mu_{0}$, this leads to the relation

$$
\zeta v(\xi)=v(\xi) \zeta_{n}
$$

from which we conclude that $\zeta=\zeta_{n} \mathbf{1}_{n}$. It then follows from (3.58) that $h(A, B)=\zeta_{n} \mathbf{1}_{n}$, and thereby (3.57) implies that $\rho=\mathbf{1}_{n}$. This contradicts our assumption that $\rho$ is a non-unit element of $S \mathbb{T}_{n}$. Therefore the $\mathbb{T}_{n-1}$-action $\hat{\Psi}^{b}$ on $P$ is effective. 
The statement of the following lemma can be obtained as an immediate consequence of Theorem 7.2 of [1] (the proof of which itself is based on results of [20]). We give here a direct proof since we shall need some details of it subsequently.

Lemma 3. The reduced phase space $P=\mu^{-1}\left(\mu_{0}\right) / G_{0}$ is connected.

Proof. It is enough to prove that any two points of $P$ can be connected by a continuous path. We fix a point $x \in P$ and define $P_{x}:=\{z \in P \mid \hat{\beta}(z)=\hat{\beta}(x)\}$, where $\hat{\beta}=\left(\hat{\beta}_{1}, \ldots, \hat{\beta}_{n}\right)$ is the $\mathbb{R}^{n}$-valued function on $P$ that descends from the $\mathbb{R}^{n}$-valued 6 invariant function $\beta$ on $\mu^{-1}\left(\mu_{0}\right)$. We pick an arbitrary $z \in P_{x}$, and next show that $x$ can be connected to $z$. To begin, denote some representatives of $x$ and $z$ in $\mu^{-1}\left(\mu_{0}\right)$ by $(A, B)$ and $\left(A^{\prime}, B^{\prime}\right)$, respectively. Referring to Section 2.2, we have $g(B) B g(B)^{-1}=\delta(\hat{\beta}(x))=g\left(B^{\prime}\right) B^{\prime} g\left(B^{\prime}\right)^{-1}$ for some $S U(n)$ matrices $g(B)$ and $g\left(B^{\prime}\right)$. We see from the proof of Theorem 2 that $g(B)$ and $g\left(B^{\prime}\right)$ can be chosen to have the same last column (in fact, one may take $g(B)_{j n}=g\left(B^{\prime}\right)_{j n}=z_{j}(\delta(\hat{\beta}(x)), y)^{\frac{1}{2}}$ defined in (3.16) $)$. Then it follows that $g(B) \mu_{0} g(B)^{-1}=g\left(B^{\prime}\right) \mu_{0} g\left(B^{\prime}\right)^{-1}$, which in turn implies that $h:=g(B)^{-1} g\left(B^{\prime}\right)$ is in $G_{0}$. This tells us that the representative of $z$ in $\mu^{-1}\left(\mu_{0}\right)$ can be replaced by $\left(A^{\prime \prime}, B\right)=\left(h A^{\prime} h^{-1}, h B^{\prime} h^{-1}\right)$. Then, it must be true that $A^{\prime \prime}=A M$ where $M=g(B)^{-1} \zeta g(B)$ for some $\zeta \in S \mathbb{T}_{n}$. This holds because the moment map constraint for $(A, B)$ and $\left(A^{\prime \prime}, B\right)$ implies that $A B A^{-1}=A^{\prime \prime} B A^{\prime \prime-1}$, and $B$ is regular by Theorem 2 . Next, by using the $\mathbb{T}_{n-1^{-}}$ action (2.30), we can rewrite the equality $\left(A^{\prime \prime}, B\right)=(A M, B)$ as $\left(A^{\prime \prime}, B\right)=\Psi_{\eta}^{b}(A, B)$, where $\eta \in \mathbb{T}_{n-1}$ is defined by the relation $\zeta^{-1}=\rho(\eta)$ with (2.28). Finally, we choose a continuous curve $[0,1] \ni s \mapsto \tau(s) \in \mathbb{T}_{n-1}$ for which $\tau(0)$ is the identity and $\tau(1)=\eta$, whereby we obtain the continuous path $\hat{\Psi}_{\tau(s)}^{b}(x)$ in $P$ that connects $x$ to $z$. Notice that $\hat{\Psi}_{\tau(s)}^{b}(x) \in P_{x}$ for all $s$, and thus we have also shown that the $\hat{\Psi}^{b}$ action (3.54) of $\mathbb{T}_{n-1}$ is transitive on $P_{x}$.

We now take two arbitrary points $x_{0}, x_{1} \in P$ for which $\hat{\beta}\left(x_{0}\right) \neq \hat{\beta}\left(x_{1}\right)$, and prove the existence of a continuous path $[0,1] \ni s \mapsto x(s) \in P$ for which $x(0)=x_{0}$ and $x(1)=x_{1}$. The following argument relies on the first part of the proof of Theorem 2 . We begin by choosing a continuous path $\xi(s) \in \mathcal{A}_{y}$ in such a way that $\xi(0)=\hat{\beta}\left(x_{0}\right)$ and $\xi(1)=\hat{\beta}\left(x_{1}\right)$. Next we define the vector function $v(s)$ by putting $v_{l}(s):=z_{l}(\delta(\xi(s)), y)^{\frac{1}{2}}$ using (3.16). Since $v(s)$ is continuous in $s$, we can find (actually could give explicitly) an $S U(n)$-valued continuous function $g(s)$ that solves $\mu_{0}=g(s)^{-1} \mu_{v(s)} g(s)$, where $\mu_{v(s)}$ is obtained by replacing $v(\xi)$ in (3.22) by $v(s)$ (see also (3.23) $)$. We continue by defining $B(s):=g(s)^{-1} \delta(\xi(s)) g(s)$, and then note the existence of a continuous function $A(s) \in S U(n)$ for which $A(s) B(s) A(s)^{-1}=\mu_{0} B(s)$. Such function exists since $B(s)$ is similar to $\mu_{0} B(s)$, as can be seen from the discussion around equations (3.21) - (3.25), and the eigenvectors of $B(s)$ and that of $\mu_{0} B(s)$ can be chosen as continuous functions of $s$. Now the projection of the curve $(A(s), B(s)) \in \mu^{-1}\left(\mu_{0}\right)$ yields a continuous curve $\tilde{x}(s):=p(A(s), B(s)) \in P$ for which $\hat{\beta}(\tilde{x}(0))=\hat{\beta}\left(x_{0}\right)$ and $\hat{\beta}(\tilde{x}(1))=\hat{\beta}\left(x_{1}\right)$. By the previous part of the proof, it is obviously possible to find a continuous curve $\tau(s) \in \mathbb{T}_{n-1}$ such that $x(s):=\hat{\Psi}_{\tau(s)}^{b}(\tilde{x}(s))$ gives the path connecting $x_{0}$ with $x_{1}$.

Remark 3. The main message of the present subsection is that the reduced phase space $(P, \hat{\omega})$ is naturally equipped with two effective Hamiltonian actions of the torus $\mathbb{T}_{n-1}$. The first is

\footnotetext{
${ }^{6}$ The fact that we here consider $\hat{\beta}$ and $\beta$ as $\mathbb{R}^{n}$-valued functions, but elsewhere view them as $\mathbb{R}^{n-1}$-valued functions should not lead to any confusion; we have $\hat{\beta}_{n} \equiv \pi-\sum_{k=1}^{n-1} \hat{\beta}_{k}$ and similarly for $\beta$.
} 
the action $\hat{\Psi}^{b}(\underline{3.54})$, which we call the $\hat{\beta}$-generated action since its moment map is given by $\hat{\beta}$ (3.56). The second is the $\hat{\alpha}$-generated action, which can be defined by the formula

$$
\hat{\Psi}_{\tau}^{a} \circ p=p \circ\left(\left.\Psi_{\tau}^{a}\right|_{\mu^{-1}\left(\mu_{0}\right)}\right), \quad \forall \tau \in \mathbb{T}_{n-1},
$$

where $\Psi^{a}$ is given by (2.29). The corresponding moment map is

$$
\hat{\alpha} \equiv\left(\hat{\alpha}_{1}, \ldots, \hat{\alpha}_{n-1}\right): P \rightarrow \mathbb{R}^{n-1}
$$

where the functions $\hat{\alpha}_{j}$ descend from the spectral Hamiltonians $\alpha_{j}$ introduced in (2.23). The data $(P, \hat{\omega}, \hat{\alpha})$ and $(P, \hat{\omega}, \hat{\beta})$ both encode Hamiltonian toric manifolds, or in other words we have two completely integrable systems on the reduced phase space $(P, \hat{\omega})$.

Remark 4. Let us introduce the following open submanifolds of $P$ :

$$
P_{0}^{a}:=\hat{\alpha}^{-1}\left(\mathcal{P}_{y}^{0}\right) \quad \text { and } \quad P_{0}^{b}:=\hat{\beta}^{-1}\left(\mathcal{P}_{y}^{0}\right)
$$

Observe from the proofs of Lemmas 2 and 3 that the $\hat{\Psi}^{b}$ action is free and transitive on $P_{x}$ for all $x \in P_{0}^{b}$ (the transitivity holds for all $x \in P$ ). Thus $P_{0}^{b}$ is a principal $\mathbb{T}_{n-1}$-bundle over the base $\mathcal{P}_{y}^{0}$. This bundle is topologically trivial since its base is contractible. Consider now $x_{0}, x_{1} \in P$ such that $x_{0} \notin P_{0}^{b}$ and $x_{1} \in P_{0}^{b}$. Then $x_{0}$ can be connected to $x_{1}$ by a curve $x(s)$ as in the proof of Lemma 3 in such a way that $\hat{\beta}(x(s)) \in \mathcal{P}_{y}^{0}$ for all $0<s \leq 1$ (since $\mathcal{P}_{y}$ is a convex polytope). This in turn implies that $P_{0}^{b}$ is a dense open submanifold of $P$. By the reasoning used below Eq. (3.27), $P_{0}^{a}$ is a also a dense open submanifold of $P$ and, equipped with the $\hat{\Psi}^{a}$ action, it is a principal $\mathbb{T}_{n-1}$-bundle over $\mathcal{P}_{y}^{0}$.

\subsection{The global structure of the reduced systems $(P, \hat{\omega}, \hat{\alpha})$ and $(P, \hat{\omega}, \hat{\beta})$}

We below identify the reduced systems by utilizing a celebrated result of Delzant [5] that characterizes Hamiltonian toric manifolds in terms of the image of the moment map.

Delzant's first theorem (Th. 2.1 of [5]). Let $\left(M_{1}, \omega_{1}\right)$ and $\left(M_{2}, \omega_{2}\right)$ be $2 d$-dimensional Hamiltonian toric manifolds with moment maps $\Phi_{1}: M_{1} \rightarrow \mathcal{T}^{*}$ and $\Phi_{2}: M_{2} \rightarrow \mathcal{T}^{*}$, where $\mathcal{T}$ is the Lie algebra of the d-dimensional torus $\mathbb{T}$ acting on $M_{1}$ and on $M_{2}$. If the images $\Phi_{1}\left(M_{1}\right)$ and $\Phi_{2}\left(M_{2}\right)$ coincide, then there exists a $\mathbb{T}$-equivariant symplectomorphism $\varphi: M_{1} \rightarrow M_{2}$ such that $\Phi_{2} \circ \varphi=\Phi_{1}$.

According to an earlier result of Atiyah, Guillemin and Sternberg, the images in question are convex polytopes. Delzant also obtained full classification of the moment polytopes associated with Hamiltonian toric manifolds, which are now routinely called Delzant polytopes [2].

By now, we have exhibited two effective Hamiltonian actions of the torus $\mathbb{T}_{n-1}=U(1)^{n-1}$ on the compact connected reduced phase space $(P, \hat{\omega})$. Referring to a fixed basit 7 of the Lie algebra $\mathcal{T}_{n-1}$ of $\mathbb{T}_{n-1}$, the respective moment maps are $\hat{\alpha}: P \rightarrow \mathbb{R}^{n-1}$ (3.65) and $\hat{\beta}: P \rightarrow \mathbb{R}^{n-1}$ (3.56). The Delzant polytopes are provided in both cases by $\mathcal{P}_{y}$ (3.11).

\footnotetext{
${ }^{7}$ Our base elements, $X_{1}, \ldots, X_{n-1}$, correspond to a fixed product structure (2.27), and realize $\mathbb{T}_{n-1}$ as $\mathcal{T}_{n-1}$ factored by the lattice $\operatorname{span}_{\mathbb{Z}}\left\{2 \pi X_{1}, \ldots, 2 \pi X_{n-1}\right\}$, i.e., the corresponding Hamiltonian flows are $2 \pi$-periodic.
} 
Specialists of symplectic geometry can immediately recognize the Delzant polytope $\mathcal{P}_{y}(3.11)$ as the one associated with a very standard Hamiltonian toric manifold: $\mathbb{C} P(n-1)$ equipped with a multiple of the Fubini-Study form and the familiar 'rotational action' of $\mathbb{T}_{n-1}$. For the sake of keeping our paper self-contained, and also since we need to fix notations, we next explain how this Hamiltonian toric manifold comes about.

Let us start with the symplectic vector space $\mathbb{C}^{n} \simeq \mathbb{R}^{2 n}$ endowed with the Darboux form

$$
\Omega_{\mathbb{C}^{n}}=\mathrm{i} \sum_{k=1}^{n} d \bar{u}_{k} \wedge d u_{k}
$$

where $u_{k}(k=1, \ldots, n)$ are the components of the vector $u$ that runs over $\mathbb{C}^{n}$. Then consider the Hamiltonian action $\psi$ of the group $U(1)$ on $\mathbb{C}^{n}$ operating as

$$
\psi_{e^{\mathrm{i} \gamma}}(u):=e^{\mathrm{i} \gamma} u
$$

This action is generated by the moment map $\chi: \mathbb{C}^{n} \rightarrow \mathbb{R}$,

$$
\chi(u) \equiv \sum_{k=1}^{n}\left|u_{k}\right|^{2}
$$

since $d \chi=\Omega_{\mathbb{C}^{n}}(V, \cdot)$ holds for the vector field $V=\mathrm{i} \sum_{k=1}^{n}\left(u_{k} \frac{\partial}{\partial u_{k}}-\bar{u}_{k} \frac{\partial}{\partial \bar{u}_{k}}\right)$ associated with the infinitesimal action. For any fixed value $\chi_{0}>0$, usual symplectic reduction yields the reduced phase space

$$
\chi^{-1}\left(\chi_{0}\right) / U(1) \equiv \mathbb{C} P(n-1)
$$

For $\chi_{0}=1$, the reduced symplectic form is the standard Fubini-Study form $\omega_{\mathrm{FS}}$ of $\mathbb{C} P(n-1)$. On the $\mathbb{C}^{n-1}$ chart corresponding to those $u \in \chi^{-1}(1)$ for which $u_{n} \neq 0$, the reduced symplectic form becomes

$$
\omega_{\mathrm{FS}}\left(\mathbb{C}^{n-1}\right)=\mathrm{i} \frac{\sum_{k=1}^{n-1} d \bar{z}_{k} \wedge d z_{k}}{1+|z|^{2}}-\mathrm{i} \frac{\sum_{j, k=1}^{n-1} z_{j} \bar{z}_{k} d \bar{z}_{j} \wedge d z_{k}}{\left(1+|z|^{2}\right)^{2}}=\mathrm{i} \bar{\partial} \partial \log \left(|z|^{2}+1\right),
$$

where we use the 'inhomogeneous coordinates' $z_{j}:=\frac{u_{j}}{u_{n}}$ and $|z|^{2} \equiv \sum_{k=1}^{n-1}\left|z_{k}\right|^{2}$. It is wellknown that $\omega_{\mathrm{FS}}$ takes the form (3.71) in terms of all the $n$ possible systems of inhomogeneous coordinates that together cover $\mathbb{C} P(n-1)$. For arbitrary $\chi_{0}>0$, one has the following result.

Lemma 4. The reduced symplectic manifold $\chi^{-1}\left(\chi_{0}\right) / U(1)$ obtained from $\left(\mathbb{C}^{n}, \Omega_{\mathbb{C}^{n}}\right)$ as described above is the complex projective space $\mathbb{C} P(n-1)$ equipped with the symplectic form $\chi_{0} \omega_{\mathrm{FS}}$.

Now focus on the action $R: \mathbb{T}_{n-1} \times \mathbb{C}^{n} \rightarrow \mathbb{C}^{n}$ of the torus $\mathbb{T}_{n-1}$ on $\mathbb{C}^{n}$ furnished by

$$
R_{\tau}\left(u_{1}, \ldots, u_{n-1}, u_{n}\right):=\left(\tau_{1} u_{1}, \ldots, \tau_{n-1} u_{n-1}, u_{n}\right), \quad \forall \tau \in \mathbb{T}_{n-1}, \forall u \in \mathbb{C}^{n} .
$$

Defining

$$
J_{k}:=\left|u_{k}\right|^{2}, \quad \forall k=1, \ldots, n-1,
$$

the corresponding moment map can be taken to be $J=\left(J_{1}, \ldots, J_{n-1}\right): \mathbb{C}^{n} \rightarrow \mathbb{R}^{n-1}$. Of course, the moment map of the torus action is unique only up to a shift by an arbitrary constant, which we shall fix by convenience. 
The above $\mathbb{T}_{n-1}$-action and moment map survive the symplectic reduction by the $U(1)$ action (3.68) and descend to the rotational $\mathbb{T}_{n-1}$-action on $\left(\mathbb{C} P(n-1), \chi_{0} \omega_{\mathrm{FS}}\right)$, which thus becomes a Hamiltonian toric manifold. This means that the rotational $\mathbb{T}_{n-1}$-action, denoted as $\mathcal{R}: \mathbb{T}_{n-1} \times \mathbb{C} P(n-1) \rightarrow \mathbb{C} P(n-1)$, operates according to the rule

$$
\mathcal{R}_{\tau} \circ \pi_{\chi_{0}}=\pi_{\chi_{0}} \circ R_{\tau}
$$

where $\pi_{\chi_{0}}: \chi^{-1}\left(\chi_{0}\right) \rightarrow \mathbb{C} P(n-1)$ is the canonical projection. We define its moment map $\mathcal{J}=\left(\mathcal{J}_{1}, \ldots, \mathcal{J}_{n-1}\right): \mathbb{C} P(n-1) \rightarrow \mathbb{R}^{n-1}$ by the formula

$$
\mathcal{J}_{k} \circ \pi_{\chi_{0}}=J_{k}+J_{k}^{0}, \quad k=1, \ldots, n-1,
$$

where the $J_{k}^{0}$ are constants. It is obvious that

$$
0 \leq J_{k} \quad \text { and } \quad \sum_{k=1}^{n-1} J_{k} \leq \chi_{0} .
$$

The point to note is that if we choose $\chi_{0}:=(\pi-|y| n)$ and $J_{k}^{0}:=|y|$, then the Delzant polytope $\mathcal{J}(\mathbb{C} P(n-1))$ of the rotational $\mathbb{T}_{n-1}$-action coincides with the polytope $\mathcal{P}_{y}$ (3.11). Therefore we obtain the following main result of this section by combining Delzant's theorem with the statements proved previously.

Theorem 3. Choose $y \in \mathbb{R}$ for which $0<|y|<\frac{\pi}{n}$. Consider the Hamiltonian toric manifold $\left(\mathbb{C} P(n-1),(\pi-|y| n) \omega_{\mathrm{FS}}, \mathcal{J}\right)$, where $\mathcal{J}$ defined by $\mathcal{J}_{k} \circ \pi_{\chi_{0}}=J_{k}+|y|$ is the moment map of the rotational $\mathbb{T}_{n-1}$-action, and consider also the Hamiltonian toric manifolds $(P, \hat{\omega}, \hat{\alpha})$ and $(P, \hat{\omega}, \hat{\beta})$ that result from the quasi-Hamiltonian reduction according to Theorem 1 and Remark 3 . Then any two of these three Hamiltonian toric manifolds are $\mathbb{T}_{n-1}$-equivariantly symplectomorphic. More precisely, there exists a diffeomorphism $\phi_{\alpha}: P \rightarrow \mathbb{C} P(n-1)$ such that

$$
\phi_{\alpha}^{*}\left((\pi-|y| n) \omega_{\mathrm{FS}}\right)=\hat{\omega}, \quad \hat{\alpha}=\mathcal{J} \circ \phi_{\alpha},
$$

and also a diffeomorphism $\phi_{\beta}: P \rightarrow \mathbb{C} P(n-1)$ such that

$$
\phi_{\beta}^{*}\left((\pi-|y| n) \omega_{\mathrm{FS}}\right)=\hat{\omega}, \quad \hat{\beta}=\mathcal{J} \circ \phi_{\beta} .
$$

The composed diffeomorphism $\phi:=\phi_{\beta}^{-1} \circ \phi_{\alpha}: P \rightarrow P$ converts $(P, \hat{\omega}, \hat{\alpha})$ into $(P, \hat{\omega}, \hat{\beta})$.

Remark 5. Theorem 3 says that both completely integrable Hamiltonian systems $(P, \hat{\omega}, \hat{\alpha})$ and $(P, \hat{\omega}, \hat{\beta})$ obtained from the quasi-Hamiltonian reduction can be identified with the system on $\left(\mathbb{C} P(n-1),(\pi-|y| n) \omega_{\mathrm{FS}}\right)$ provided by the simple Hamiltonians $\mathcal{J}_{k}$ that generate the rotational action of $\mathbb{T}_{n-1}$. As we shall see later, the functions $\mathcal{J}_{k} \in C^{\infty}(\mathbb{C} P(n-1))$ play the role of particlepositions in the compactified $\mathrm{III}_{\mathrm{b}}$ system [31]. It will turn out that if one converts $\hat{\beta}$ into the particle-positions $\mathcal{J}$ of the $\mathrm{III}_{\mathrm{b}}$ system by the symplectomorphism $\phi_{\beta}$, then $\hat{\alpha}$ is converted by the same symplectomorphism into the action-variables of the system. Roughly speaking, the exchange of the roles of $\hat{\alpha}$ and $\hat{\beta}$ will then explain the Ruijsenaars self-duality since the other symplectomorphism $\phi_{\alpha}$ converts $\hat{\alpha}$ into the particle-positions and $\hat{\beta}$ into the action-variables. From now on, the symplectomorphisms $\phi_{\alpha}, \phi_{\beta}$ that appear in Theorem 3 as well as their inverses 
and compositions will be referred to as Delzant symplectomorphisms, or simply as Delzant maps. In the next section, we explicitly construct the Delzant maps

$$
f_{\alpha}:=\phi_{\alpha}^{-1} \quad \text { and } \quad f_{\beta}:=\phi_{\beta}^{-1}
$$

which will be utilized in Section 5 where the statements of this remark will be elaborated.

\section{Construction of the Delzant symplectomorphisms}

The aim of this section is to construct explicitly the Delzant maps $f_{\alpha}, f_{\beta}: \mathbb{C} P(n-1) \rightarrow P$ whose existence has been established by Theorem 3. We shall see that the Ruijsenaars-Schneider Lax matrix $L_{y}^{\text {loc }}$ appears as a principal building block of these symplectomorphisms. This remarkable fact will be further exploited in Section 5 where the emergence of the compactified $\mathrm{III}_{\mathrm{b}}$ system as the fruit of the quasi-Hamiltonian reduction will be established and the Ruijsenaars self-duality map of the $\mathrm{III}_{\mathrm{b}}$ system will be expressed in terms of the Delzant maps.

From the technical point of view, below we first describe a local version of the map $f_{\beta}$ defined in some dense open subset of $\mathbb{C} P(n-1)$, and then we construct its global extension that will involve the global extension of the local Lax matrix (appearing already in [31]). Finally, we shall construct $f_{\alpha}$ out of $f_{\beta}$ and certain involutions.

\subsection{Local version of the Delzant map $f_{\beta}$}

Let us denote by $\mathbb{C} P(n-1)_{0}$ the dense open submanifold of $\mathbb{C} P(n-1)=\chi^{-1}(\pi-n|y|) / U(1)$ where none of the $n$ homogeneous coordinates $u_{k}$ can vanish (cf. Eq. (3.70)). In what follows we construct a symplectomorphism

$$
f_{0}: \mathbb{C} P(n-1)_{0} \rightarrow P_{0}^{b},
$$

where $P_{0}^{b}=\hat{\beta}^{-1}\left(\mathcal{P}_{y}^{0}\right)$ is the dense open submanifold of $P$ introduced in (3.66) .

On the subset of the constraint surface $\chi^{-1}(\pi-n|y|)$ covering $\mathbb{C} P(n-1)_{0}$ we may impose the gauge fixing condition $u_{n}>0$, and then $u_{j}(j=1, \ldots, n-1)$ parametrize $\mathbb{C} P(n-1)_{0}$. Adopting this condition, we now introduce Darboux coordinates $\xi_{j}, \tau_{j}(j=1, \ldots, n-1)$ on $\mathbb{C} P(n-1)_{0}$ by setting $u_{j}:=\tau_{j} \sqrt{\xi_{j}-|y|}$ for $j=1, \ldots, n-1$, where $\xi \in \mathcal{P}_{y}^{0}(\underline{3.11}), \tau \in \mathbb{T}_{n-1}$. That is we parametrize $\mathbb{C} P(n-1)_{0}$ using the diffeomorphism

$$
\mathcal{E}: \mathcal{P}_{y}^{0} \times \mathbb{T}_{n-1} \rightarrow \mathbb{C} P(n-1)_{0}
$$

given by

$$
\mathcal{E}(\xi, \tau):=\pi_{\chi_{0}}\left(\tau_{1} \sqrt{\xi_{1}-|y|}, \ldots, \tau_{n-1} \sqrt{\xi_{n-1}-|y|}, \sqrt{\xi_{n}-|y|}\right) \quad \text { with } \quad \xi_{n} \equiv \pi-\sum_{k=1}^{n-1} \xi_{k}
$$

and $\pi_{\chi_{0}}$ defined in Subsection 3.4. An easy calculation shows that the Fubini-Study symplectic structure on $\mathbb{C} P(n-1)_{0}$ takes the Darboux form in the variables $\xi_{j}, \tau_{j}$. Speaking more precisely, 
with the parametrization $\tau_{k}:=e^{\mathrm{i} \theta_{k}}(k=1, \ldots, n-1)$, there holds the relation

$$
(\pi-n|y|) \mathcal{E}^{*}\left(\omega_{\mathrm{FS}}\right)=\mathrm{i} \sum_{k=1}^{n-1} d \xi_{k} \wedge d \tau_{k} \tau_{k}^{-1}=\sum_{k=1}^{n-1} d \theta_{k} \wedge d \xi_{k} .
$$

As further pieces of preparation, recall the isomorphism $\rho: \mathbb{T}_{n-1} \rightarrow S \mathbb{T}_{n}(2.28)$,

$$
\rho(\tau) \equiv \exp \left(\mathrm{i} \sum_{j=1}^{n-1} \theta_{j}\left(E_{j, j}-E_{j+1, j+1}\right)\right) \quad \text { for } \quad \tau=\left(e^{\mathrm{i} \theta_{1}}, \ldots, e^{\mathrm{i} \theta_{n-1}}\right),
$$

and consider the vector

$$
v_{j}(\xi, y):=\left[\frac{\sin y}{\sin n y}\right]^{\frac{1}{2}} W_{j}(\delta(\xi), y), \quad \forall \xi \in \mathcal{P}_{y}, \quad j=1, \ldots, n,
$$

with $W_{j}$ in (1.5) where non-negative square roots are taken. Observe that if $\xi \in \mathcal{P}_{y}^{0}$, then all $v_{j}(\xi, y)$ are strictly positive since their squares are the same as the right-hand side of (3.16). It is also important to notice that these are $C^{\infty}$ functions on the open alcove $\mathcal{P}_{y}^{0}$, but their first derivatives develop some singularities at the boundary of $\mathcal{P}_{y}^{0}$.

It is readily checked that the following formulae yield a unitary matrix $g(v) \in U(n)$ for any vector $v \in \mathbb{R}^{n}$ that has unit norm and component $v_{n} \neq-1$ :

$$
\begin{aligned}
& g(v)_{j n}:=-g(v)_{n j}:=v_{j}, \quad \forall j=1, \ldots, n-1, \quad g(v)_{n n}:=v_{n}, \\
& g(v)_{j l}:=\delta_{j l}-\frac{v_{j} v_{l}}{1+v_{n}}, \quad \forall j, l=1, \ldots, n-1 .
\end{aligned}
$$

Equations (1.5), (3.16)-(3.19) imply that the vector $v(\xi, y)$ in (4.6) has unit norm, and using this we now introduce the unitary (actually real-orthogonal) matrix $g_{y}(\xi) \in U(n)$ by setting

$$
g_{y}(\xi):=g(v(\xi, y)), \quad \forall \xi \in \mathcal{P}_{y} .
$$

Theorem 4. We can define a map $f_{0}: \mathbb{C} P(n-1)_{0} \rightarrow P_{0}^{b}$ by the formula

$$
\left(f_{0} \circ \mathcal{E}\right)(\xi, \tau):=p\left(g_{y}(\xi)^{-1} L_{y}^{\mathrm{loc}}\left(\delta(\xi), \rho(\tau)^{-1}\right) g_{y}(\xi), g_{y}(\xi)^{-1} \delta(\xi) g_{y}(\xi)\right),
$$

where $L_{y}^{\text {loc }}$ is the Lax matrix given by 1.4) and $p: \mu^{-1}\left(\mu_{0}\right) \rightarrow P$ is the canonical projection. This map is a symplectic diffeomorphism with respect to the restricted symplectic forms,

$$
f_{0}^{*}(\hat{\omega})=(\pi-n|y|) \omega_{\mathrm{FS}}
$$

and it intertwines the restrictions of the corresponding toric moment maps,

$$
f_{0}^{*}(\hat{\beta})=\mathcal{J} \text {. }
$$

The hardest part of the proof will be the verification of Eq. (4.10), and before dealing with this we present two lemmas. 
Lemma 5. The $S U(n)$ matrix $L_{y}^{\operatorname{loc}}(\delta(\xi), \Theta)$ given by Eq. 1.4) verifies the relation

$$
L_{y}^{\mathrm{loc}}(\delta(\xi), \Theta) \delta(\xi) L_{y}^{\mathrm{loc}}(\delta(\xi), \Theta)^{-1}=\left[e^{2 \mathrm{i} y} \mathbf{1}_{n}+\left(e^{2 \mathrm{i}(1-n) y}-e^{2 \mathrm{i} y}\right) v(\xi, y) v(\xi, y)^{\dagger}\right] \delta(\xi)
$$

for all $\xi \in \mathcal{P}_{y}^{0}$ and $\Theta \in S \mathbb{T}_{n}$, with the vector 4.6.).

Proof. We know from the proof of Theorem 2 (cf. the discussion around Eqs. (3.28) and (3.30) ) that the unitary matrices $\delta(\xi)$ and $\left[e^{2 \mathrm{i} y} \mathbf{1}_{n}+\left(e^{2 \mathrm{i}(1-n) y}-e^{2 \mathrm{i} y}\right) v(\xi, y) v(\xi, y)^{\dagger}\right] \delta(\xi)$ have the same spectra, and hence there exists a unitary matrix $N(\xi, y)$ such that

$$
N(\xi, y) \delta(\xi) N(\xi, y)^{-1}=\left[e^{2 \mathrm{i} y} \mathbf{1}_{n}+\left(e^{2 \mathrm{i}(1-n) y}-e^{2 \mathrm{i} y}\right) v(\xi, y) v(\xi, y)^{\dagger}\right] \delta(\xi) .
$$

By conjugating the last relation by $N(\xi, y)^{-1}$ we obtain

$$
\delta(\xi)=\left[e^{2 \mathrm{i} y} \mathbf{1}_{n}+\left(e^{2 \mathrm{i}(1-n) y}-e^{2 \mathrm{i} y}\right) N(\xi, y)^{-1} v(\xi, y)\left(N(\xi, y)^{-1} v(\xi, y)\right)^{\dagger}\right] N(\xi, y)^{-1} \delta(\xi) N(\xi, y)
$$

and by inverting the term in square brackets we arrive at

$$
N(\xi, y)^{-1} \delta(\xi) N(\xi, y)=\left[e^{-2 \mathrm{i} y} \mathbf{1}_{n}+\left(e^{2 \mathrm{i}(n-1) y}-e^{-2 \mathrm{i} y}\right) N(\xi, y)^{-1} v(\xi, y)\left(N(\xi, y)^{-1} v(\xi, y)\right)^{\dagger}\right] \delta(\xi) .
$$

Let us rewrite Eq. (4.13) for $-y$ as

$$
N(\xi,-y) \delta(\xi) N(\xi,-y)^{-1}=\left[e^{-2 \mathrm{i} y} \mathbf{1}_{n}+\left(e^{2 \mathrm{i}(n-1) y}-e^{-2 \mathrm{i} y}\right) v(\xi,-y) v(\xi,-y)^{\dagger}\right] \delta(\xi) .
$$

We have learned in proving Theorem 2 (cf. Eq. (3.33) ) that the equality of the spectra of the matrices $\delta(\xi)$ and $\left[e^{2 \mathrm{i} y} \mathbf{1}_{n}+\left(e^{2 \mathrm{i}(1-n) y}-e^{2 \mathrm{i} y}\right) w w^{\dagger}\right] \delta(\xi)$ fixes the absolute values of the components of the vector $w$ to be given by the right-hand side of (4.6). Comparing (4.16) with (4.15), we therefore see that the absolute values of the components of the vector $N(\xi, y)^{-1} v(\xi, y)$ are the same as the (strictly positive) components of the vector $v(\xi,-y)$. Note that the matrix $N(\xi, y)$ verifying Eq. (4.13) is not unique because it can be multiplied from the right by any diagonal element of $U(n)$ while keeping (4.13) valid. However, this ambiguity can be completely fixed by requiring that the vector $N(\xi, y)^{-1} v(\xi, y)$ has all components real and strictly positive. We denote the unique matrix $N(\xi, y)$ satisfying this requirement by $\tilde{N}(\xi, y)$. Thus we have

$$
v(\xi, y)=\tilde{N}(\xi, y) v(\xi,-y) \quad \text { and hence } \quad \tilde{N}(\xi,-y)=\tilde{N}(\xi, y)^{-1}
$$

By considering it for $N(\xi, y):=\tilde{N}(\xi, y)$, let us rewrite (4.13) as

$$
\tilde{N}(\xi, y) \delta(\xi)-e^{2 \mathrm{i} y} \delta(\xi) \tilde{N}(\xi, y)=\left(e^{2 \mathrm{i}(1-n) y}-e^{2 \mathrm{i} y}\right) v(\xi, y)\left(\tilde{N}(\xi, y)^{-1} v(\xi, y)\right)^{\dagger} \tilde{N}(\xi, y)^{-1} \delta(\xi) \tilde{N}(\xi, y) .
$$

With the help of (4.17), we can further rewrite the last relation as

$$
\tilde{N}(\xi, y) \delta(\xi)-e^{2 \mathrm{i} y} \delta(\xi) \tilde{N}(\xi, y)=\left(e^{2 \mathrm{i}(1-n) y}-e^{2 \mathrm{i} y}\right) v(\xi, y) v(\xi,-y)^{\dagger} \tilde{N}(\xi,-y) \delta(\xi) \tilde{N}(\xi,-y)^{-1}
$$

Expressing $\tilde{N}(\xi,-y) \delta(\xi) \tilde{N}(\xi,-y)^{-1}$ from Eq. (4.16) and using subsequently Eq. (3.19), we derive

$$
\tilde{N}(\xi, y) \delta(\xi)-e^{2 \mathrm{i} y} \delta(\xi) \tilde{N}(\xi, y)=\left(1-e^{2 n \mathrm{i} y}\right) v(\xi, y) v(\xi,-y)^{\dagger} \delta(\xi)
$$


By solving this for the components of $\tilde{N}(\xi, y)$ we obtain the equality

$$
\tilde{N}(\xi, y)=e^{\mathrm{i}(n-1) y} L_{y}^{\mathrm{loc}}\left(\delta(\xi), \mathbf{1}_{n}\right)
$$

This implies the desired relation (4.12). The above argument also shows that $L_{y}^{\text {loc }}(\delta(\xi), \Theta)$ is unitary, and the fact that its determinant equals 1 is easily checked by the determinant formula of Cauchy matrices.

Lemma 6. Every element $(A, B) \in \mu^{-1}\left(\mu_{0}\right)$ such that $p(A, B)$ belongs to $P_{0}^{b}$ (3.66) has the form

$$
(A, B)=\Psi_{\left(g_{y}(\xi) \eta\right)^{-1}}\left(L_{y}^{\mathrm{loc}}\left(\delta(\xi), \rho(\tau)^{-1}\right), \delta(\xi)\right)
$$

with $\xi=\beta(A, B), \tau \in \mathbb{T}_{n-1}$ and $\eta \in U(n)$ for which $\eta^{-1} \mu_{0} \eta=\mu_{0}$, using the notation (2.10). By this formula, the pair $(\xi, \tau) \in \mathcal{P}_{y}^{0} \times \mathbb{T}_{n-1}$ uniquely parametrizes the projection $p(A, B) \in P_{0}^{b}$.

Proof. Conjugating the relation (4.12) by $g_{y}(\xi)^{-1}$, and putting $\Theta:=\rho(\tau)^{-1}$ (4.5), we conclude by using Eq. (3.23) that the pair (4.22) belongs to the constraint surface $\mu^{-1}\left(\mu_{0}\right)$. It follows by tracing the definitions that, after the projection $p, \tau$ parametrizes a $\mathbb{T}_{n-1}$ orbit in $P_{0}^{b}$ under the $\hat{\Psi}^{b}$-action. As was noted in Remark 4 , this action is transitive and free on $P_{0}^{b}$. Hence the above solution of the constraint (3.1) projects to the most general element of the reduced phase space $P$ for which the value of the function $\hat{\beta}$ equals $\xi$, and specifying the pair $(\xi, \tau)$ is equivalent to specifying the projection $p(A, B) \in P_{0}^{b}$.

Proof of Theorem 4: It follows directly from Lemma 6 that the formula

$$
\left(F_{0} \circ \mathcal{E}\right)(\xi, \tau):=\left(g_{y}(\xi)^{-1} L_{y}^{\mathrm{loc}}\left(\delta(\xi), \rho(\tau)^{-1}\right) g_{y}(\xi), g_{y}(\xi)^{-1} \delta(\xi) g_{y}(\xi)\right)
$$

defines a smooth map

$$
F_{0}: \mathbb{C} P(n-1)_{0} \rightarrow p^{-1}\left(P_{0}^{b}\right)
$$

which is injective and its image intersects every gauge orbit in $p^{-1}\left(P_{0}^{b}\right)$ precisely in one point. Thus $f_{0}=p \circ F_{0}: \mathbb{C} P(n-1)_{0} \rightarrow P_{0}^{b}$ is an injective and surjective smooth map. On account of (4.10) (proved in what follows), the corresponding Jacobian determinant cannot vanish and hence $f_{0}$ is a diffeomorphism.

Since the validity of (4.11) is obvious, it remains to show that $f_{0}$ satisfies (4.10). Because of Eqs. (2.7) and (4.4), this amounts to proving that the restriction of the quasi-Hamiltonian 2-form $\omega$ on $p^{-1}\left(P_{0}^{b}\right)$ pulled back by the map $F_{0} \circ \mathcal{E}$ on $\mathcal{P}_{y}^{0} \times \mathbb{T}_{n-1}$ is the Darboux 2-form:

$$
\left.\left(F_{0} \circ \mathcal{E}\right)^{*} \omega\right|_{p^{-1}\left(P_{0}^{b}\right)}=\mathrm{i} \sum_{k=1}^{n-1} d \xi_{k} \wedge d \tau_{k} \tau_{k}^{-1}
$$

In fact, we here verify this by direct computation, by inserting the formula (4.23) into the formula (2.11):

$$
\left.\left(F_{0} \circ \mathcal{E}\right)^{*} \omega\right|_{p^{-1}\left(P_{0}^{b}\right)}=\frac{1}{2}\left\langle A_{\xi, \tau}^{-1} d A_{\xi, \tau} \hat{,} d B_{\xi} B_{\xi}^{-1}\right\rangle+\frac{1}{2}\left\langle d A_{\xi, \tau} A_{\xi, \tau}^{-1} \wedge B_{\xi}^{-1} d B_{\xi}\right\rangle
$$


where $A_{\xi, \tau}:=g_{y}(\xi)^{-1} L_{y}^{\text {loc }}\left(\delta(\xi), \rho(\tau)^{-1}\right) g_{y}(\xi)$ and $B_{\xi}:=g_{y}(\xi)^{-1} \delta(\xi) g_{y}(\xi)$. Note that we have omitted in (4.26) the third term of the form $\omega$ displayed in (2.11). This term does not contribute since, due to the moment map constraint (3.1), we have $\mu_{0} B_{\xi} A_{\xi, \tau}=A_{\xi, \tau} B_{\xi}$ and hence

$$
\left\langle\left(A_{\xi, \tau} B_{\xi}\right)^{-1} d\left(A_{\xi, \tau} B_{\xi}\right) \wedge\left(B_{\xi} A_{\xi, \tau}\right)^{-1} d\left(B_{\xi} A_{\xi, \tau}\right)\right\rangle=\left\langle\left(B_{\xi} A_{\xi, \tau}\right)^{-1} d\left(B_{\xi} A_{\xi, \tau}\right) \wedge\left(B_{\xi} A_{\xi, \tau}\right)^{-1} d\left(B_{\xi} A_{\xi, \tau}\right),\right.
$$

which vanishes as the scalar product is symmetric and the wedge product is anti-symmetric.

In the following calculation, we set for simplicity $g \equiv g_{y}(\xi), L \equiv L_{y}^{\text {loc }}\left(\delta(\xi), \rho(\tau)^{-1}\right), \rho \equiv \rho(\tau)$ and $\delta \equiv \delta(\xi)$. Thus we obtain from (4.26)

$$
\left.\left(F_{0} \circ \mathcal{E}\right)^{*} \omega\right|_{p^{-1}\left(P_{0}^{b}\right)}=\frac{1}{2}\left\langle L^{-1} d L+d g g^{-1}-L^{-1} d g g^{-1} L \wedge d \delta \delta^{-1}+\delta d g g^{-1} \delta^{-1}-d g g^{-1}\right\rangle-(L \leftrightarrow \delta),
$$

where $(L \leftrightarrow \delta)$ means the first term on the r.h.s. of (4.28) with the role of $L$ and $\delta$ interchanged. By using the invariance of the scalar product $\langle.,$.$\rangle and the fact that \langle\phi \hat{,} \psi\rangle=-\langle\psi \hat{,} \phi\rangle$ for any $s u(n)$-valued differential forms $\phi$ and $\psi$, we can rewrite Eq. (4.28) as

$$
\left.\left(F_{0} \circ \mathcal{E}\right)^{*} \omega\right|_{p^{-1}\left(P_{0}^{b}\right)}=\frac{1}{2}\left\langle\delta^{-\frac{1}{2}}\left(L^{-1} d L-L^{-1} d g g^{-1} L\right) \delta^{\frac{1}{2}}+\delta^{\frac{1}{2}}\left(d L L^{-1}+L d g g^{-1} L^{-1}\right) \delta^{-\frac{1}{2}} \hat{,} \kappa+\kappa^{t}\right\rangle .
$$

Here we have introduced the $s u(n)$-valued differential form $\kappa$ by

$$
\kappa:=\frac{1}{2} d \delta \delta^{-1}+\delta^{\frac{1}{2}} d g g^{-1} \delta^{-\frac{1}{2}},
$$

$\kappa^{t}$ denotes the transposed matrix, and by using (2.20) we have $\delta^{\frac{1}{2}} \equiv \exp \left(-\mathrm{i} \sum_{k=1}^{n-1} \xi_{k} \lambda_{k}\right)$.

We can write the matrix $L$ as

$$
L \equiv L_{1}(\xi) \rho(\tau)^{-1}
$$

Thus in Eq. (4.29) the dependence of the form $\left.\left(F_{0} \circ \mathcal{E}\right)^{*} \omega\right|_{p^{-1}\left(P_{0}^{b}\right)}$ on the variable $\tau$ is hidden in the (diagonal) matrix $\rho(\tau)$. It will be convenient to employ also the decomposition

$$
\left.\left(F_{0} \circ \mathcal{E}\right)^{*} \omega\right|_{p^{-1}\left(P_{0}^{b}\right)} \equiv V+\hat{V}
$$

where $V$ depends on $\rho$ differentially, i.e., $V$ collects the terms that contain $d \rho$. The part $V$ is easily singled out from (4.29) as

$$
V=-\frac{1}{2}\left\langle d \rho \rho^{-1} \wedge d \delta \delta^{-1}+\delta d g g^{-1} \delta^{-1}-d g g^{-1}+L_{1}^{-1}\left(d \delta \delta^{-1}+d g g^{-1}-\delta^{-1} d g g^{-1} \delta\right) L_{1}\right\rangle .
$$

For $g$ is real orthogonal, $d g g^{-1}$ is anti-symmetric. Because the trace of the product of a symmetric matrix with an anti-symmetric one vanishes, we obtain

$$
\left\langle d \rho \rho^{-1} \wedge d g g^{-1}\right\rangle=0
$$

and then $V$ can be rewritten as

$$
V=-\frac{1}{2}\left\langle d \rho \rho^{-1} \hat{\prime} d \delta \delta^{-1}+L_{1}^{-1} d g g^{-1} L_{1}-L_{1}^{-1} d L_{1}+\left(\delta L_{1}\right)^{-1} d\left(\delta L_{1}\right)-\left(\delta L_{1}\right)^{-1} d g g^{-1}\left(\delta L_{1}\right)\right\rangle
$$


Now note that the constraint (3.1) implies

$$
\delta L_{1}=\zeta^{-1} L_{1} \delta, \quad \zeta:=g \mu_{0} g^{-1} .
$$

Inserting this in the last two terms of (4.35) gives directly the Darboux form:

$$
V=-\left\langle d \rho \rho^{-1} \wedge d \delta \delta^{-1}\right\rangle-\frac{1}{2}\left\langle d \rho \rho^{-1}-\delta d \rho \rho^{-1} \delta^{-1} \wedge L_{1}^{-1} d \zeta \zeta^{-1} L_{1}-L_{1}^{-1} d L_{1}\right\rangle=-\left\langle d \rho \rho^{-1} \hat{,} d \delta \delta^{-1}\right\rangle .
$$

The restricted form $\left.\omega\right|_{p^{-1}\left(P_{0}^{b}\right)}$ is closed because $p^{-1}\left(P_{0}^{b}\right)$ is a subset of a level set of the moment map, and thus its pull-back (4.32) is closed as well. Moreover, the Darboux differential form $V$ is also closed. Then we observe that the part $\hat{V}$ in (4.32) cannot depend on $\rho$ because otherwise it would not be closed. By taking this into account, (4.29) gives

$$
\hat{V}=\frac{1}{2}\left\langle\delta^{-\frac{1}{2}}\left(L_{1}^{-1} d L_{1}-L_{1}^{-1} d g g^{-1} L_{1}\right) \delta^{\frac{1}{2}}+\delta^{\frac{1}{2}}\left(d L_{1} L_{1}^{-1}+L_{1} d g g^{-1} L_{1}^{-1}\right) \delta^{-\frac{1}{2}} \hat{,} \kappa+\kappa^{t}\right\rangle .
$$

Now we again use the constraint (4.36) to derive

$$
L_{1}^{-1} d L_{1}-L_{1}^{-1} d g g^{-1} L_{1}=\delta\left(L_{1}^{-1} d L_{1}+L_{1}^{-1}\left(\delta^{-1} d \delta-\delta^{-1} d g g^{-1} \delta\right) L_{1}\right) \delta^{-1}-d \delta \delta^{-1} .
$$

Inserting the expression (4.39) into (4.38) yields immediately

$$
\hat{V}=\frac{1}{2}\left\langle L_{1}^{-1} d L_{1}+L_{1}^{-1}\left(\delta^{-1} d \delta-\delta^{-1} d g g^{-1} \delta\right) L_{1}+d L_{1} L_{1}^{-1}+L_{1} d g g^{-1} L_{1}^{-1} \hat{,} \delta^{-\frac{1}{2}}\left(\kappa+\kappa^{t}\right) \delta^{\frac{1}{2}}\right\rangle .
$$

Coming back to the formula (1.4), we notice that the unitary matrix $L_{1}$ can be cast as

$$
L_{1}=\delta^{-\frac{1}{2}} \mathcal{M} \delta^{\frac{1}{2}}
$$

where $\mathcal{M}$ is a real orthogonal matrix. With this representation of $L_{1}$, the expression $\hat{V}$ can be rewritten as

$$
\hat{V}=\frac{1}{2}\left\langle d \mathcal{M} \mathcal{M}^{-1}+\mathcal{M}^{-1} d \mathcal{M}+\mathcal{M} \kappa \mathcal{M}^{-1}+\mathcal{M}^{-1} \kappa^{t} \mathcal{M} \wedge \kappa+\kappa^{t}\right\rangle .
$$

By using again that the trace of the product of a symmetric matrix with an anti-symmetric one vanishes, and using also the invariance of the scalar product and that $\langle\phi \hat{,} \psi\rangle=\left\langle\phi^{t} \hat{,} \psi^{t}\right\rangle$ for any $s u(n)$-valued forms, the last equation implies that $\hat{V}=0$. Having calculated $V$ and $\hat{V}$ in (4.32), we finally obtain the desired equality:

$$
\left.\left(F_{0} \circ \mathcal{E}\right)^{*} \omega\right|_{p^{-1}\left(P_{0}^{b}\right)}=-\left\langle d \rho \rho^{-1} \wedge d \delta \delta^{-1}\right\rangle=\mathrm{i} \sum_{k=1}^{n-1} d \xi_{k} \wedge d \tau_{k} \tau_{k}^{-1} .
$$

Remark 6. We know from the theory of the quasi-Hamiltonian reduction that the reduced spectral Hamiltonians $\hat{\alpha}_{i}$ Poisson commute, and Theorem 4 permits to identify the $\hat{\alpha}_{i}$ on $P_{0}^{b}$ with the spectral functions of the Ruijsenaars-Schneider Lax matrix. The proof of Theorem 4 shows that this commutativity property of the $\hat{\alpha}_{i}$ can be viewed as a consequence of the 
Darboux form of the reduced symplectic structure. The fact that the spectral invariants of the Ruijsenaars-Schneider Lax matrix Poisson commute with respect to the Darboux structure was also proved previously by means of different methods (see [33, 32, 25] and references therein).

It follows easily from Theorem 4 that the local Delzant map $f_{0}$ converts $\hat{\beta}$ into particleposition variables and converts $\hat{\alpha}$ into action-variables of the local $\mathrm{III}_{\mathrm{b}}$ system. Consequently, the full reduced phase space $P$ must carry a completion of the local $\mathrm{III}_{\mathrm{b}}$ system. Eventually this completion will be identified with the one introduced by Ruijsenaars, but before explaining this further effort is needed in order to work out certain details of our picture that will enable us to give precise comparison with the results of [31] regarding also the self-duality of the completed $\mathrm{III}_{\mathrm{b}}$ system. In particular, we need to prove that $f_{0}$ extends to a global Delzant map.

\subsection{Global extension of the Lax matrix}

The local Lax matrix $L_{y}^{\text {loc }}\left(\delta(\xi), \rho(\tau)^{-1}\right)$, viewed as a function on $\mathbb{C} P(n-1)_{0}$, is the crucial ingredient of the local Delzant map of Theorem 4. The global Delzant map that we shall construct later will involve an extension of (a conjugate of) this Lax matrix to a smooth function on $\mathbb{C} P(n-1)$. We here present this extension, which appears also in [31]. In order to save space, from now on we assume that

$$
y>0 .
$$

We continue to realize $\mathbb{C} P(n-1)$ as the factor space $\mathbb{C} P(n-1)=S_{\chi_{0}}^{2 n-1} / U(1)$ with

$$
S_{\chi_{0}}^{2 n-1}=\left\{\left.\left(u_{1}, \ldots, u_{n}\right) \in \mathbb{C}^{n}\left|\sum_{k=1}^{n}\right| u_{k}\right|^{2}=\chi_{0}\right\}, \quad \chi_{0}=\pi-n|y| .
$$

In the subsequent arguments we identify the $U(1)$-invariant functions defined on $S_{\chi_{0}}^{2 n-1}$ by

$$
r_{i}:=\left|u_{i}\right| \quad \text { and } \quad \xi_{i}:=\left|u_{i}\right|^{2}+|y|, \quad i=1, \ldots, n,
$$

as functions on $\mathbb{C} P(n-1)$. Regarded in this way, $\xi_{i}$ belongs to $C^{\infty}(\mathbb{C} P(n-1))$, while $r_{i}$ is not even differentiable at its zero locus. (For $i=1, \ldots, n-1$, the function $\xi_{i}$ is just another name for the moment map component $\mathcal{J}_{i}$.)

Now we give a simple technical lemma, whose proof contains the essential observation that will lead to the global Lax matrix. Its statement will be utilized also in Subsection 4.3.

Lemma 7. By combining equations (1.5), (2.19) and (4.46), with $0<y<\frac{\pi}{n}$, consider the expressions $W_{k}(\delta(\xi), \pm y)$ as functions on $\mathbb{C} P(n-1)$. Then $W_{k}(\delta(\xi), y)$ can be written as

$$
W_{k}(\delta(\xi), y)=r_{k} w_{k}^{y}(\xi)
$$

where $w_{k}^{y}(\xi)$ represents a positive $C^{\infty}$ function on $\mathbb{C} P(n-1)$ for each $k=1, \ldots, n$. Similarly,

$$
W_{k}(\delta(\xi),-y)=r_{k-1} w_{k}^{-y}(\xi), \quad r_{0}:=r_{n},
$$

where the function $w_{k}^{-y}$ has the same properties as those mentioned for $w_{k}^{y}$. 
Proof. First restricting to $\mathbb{C} P(n-1)_{0}$ where $r_{k} \neq 0$, we directly spell out $W_{k}(\delta(\xi), y)$ in the form (4.47) with

$$
w_{k}^{y}(\xi)=\left[\frac{\sin \left(r_{k}^{2}\right)}{r_{k}^{2} \sin \left(\xi_{k}\right)}\right]^{\frac{1}{2}} R_{k}^{y}(\xi)
$$

where, introducing the shorthand $\xi_{i, l}:=\sum_{m=i}^{l} \xi_{m}$ for all $1 \leq i \leq l \leq n$, we have

$$
R_{k}^{y}(\xi)=\left(\prod_{1 \leq j \leq k-1}\left[\frac{\sin \left(\xi_{j, k-1}+y\right)}{\sin \left(\xi_{j, k-1}\right)}\right]^{\frac{1}{2}}\right)\left(\prod_{k+2 \leq j \leq n}\left[\frac{\sin \left(\xi_{k, j-1}-y\right)}{\sin \left(\xi_{k, j-1}\right)}\right]^{\frac{1}{2}}\right) .
$$

By using (4.46), it is easily checked that all arguments of the sinus-functions involved in (4.50) lie strictly in the interval $(0, \pi)$ even when running over the full $\mathbb{C} P(n-1)$, which immediately implies that $R_{k}^{y}(\xi)$ represents a positive $C^{\infty}$-function on $\mathbb{C} P(n-1)$. Since the function $\sin x / x$ remains smooth and positive at $x=0$, and $y \leq \xi_{k} \leq \pi-(n-1) y$, we then see from (4.49) that $w_{k}^{y}(\xi)$ also represents a positive $C^{\infty}$ function on $\mathbb{C} P(n-1)$. The claim about $W_{k}(\delta(\xi),-y)$ can be verified in an analogous manner.

Next, using $L_{y}^{\text {loc }}$ given in (1.4), we introduce the functions $\Lambda_{k, l}^{y}(\xi)(1 \leq k, l \leq n)$ by the equations

$$
\begin{gathered}
r_{k} r_{l-1} \Lambda_{k, l}^{y}(\xi) \equiv L_{y}^{\mathrm{loc}}\left(\delta(\xi), \mathbf{1}_{n}\right)_{k, l} \quad \text { for } \quad l \neq k+1, \quad(k, l) \neq(n, 1), \\
\Lambda_{k, k+1}^{y}(\xi) \equiv L_{y}^{\mathrm{loc}}\left(\delta(\xi), \mathbf{1}_{n}\right)_{k, k+1}, \quad \Lambda_{n, 1}^{y}(\xi) \equiv L_{y}^{\mathrm{loc}}\left(\delta(\xi), \mathbf{1}_{n}\right)_{n, 1} .
\end{gathered}
$$

These equations directly define $\Lambda_{k, l}^{y}$ as functions on $\mathbb{C} P(n-1)_{0}$, where all $r_{i}$ are non-zero. Then, by using Lemma 7 and a similar analysis for the denominators in the formula (1.4), we find that $\Lambda_{k, l}^{y}(\xi)$ extends to a $C^{\infty}$ function on $\mathbb{C} P(n-1)$ for each $1 \leq k, l \leq n$. The extended function, which we denote by the same letter, vanishes nowhere on $\mathbb{C} P(n-1)$. The last statement follows by easy inspection, and will be utilized later.

Lemma 8. By using the above functions $\Lambda_{k, l}^{y}$ and (4.46), and setting $u_{0}:=u_{n}$, we can define $C^{\infty}$ functions $L_{k, l}^{y}$ on $S_{\chi_{0}}^{2 n-1}$ by the formulae

$$
\begin{gathered}
L_{k, l}^{y}(u):=\bar{u}_{k} u_{l-1} \Lambda_{k, l}^{y}(\xi) \quad \text { for } \quad l \neq k+1, \quad(k, l) \neq(n, 1), \\
L_{k, k+1}^{y}(u):=\Lambda_{k, k+1}^{y}(\xi), \quad L^{y}(u)_{n, 1}:=\Lambda_{n, 1}^{y}(\xi) .
\end{gathered}
$$

The functions $L_{k, l}^{y}$ are $U(1)$-invariant and thus yield $C^{\infty}$ functions on $\mathbb{C} P(n-1)$ that together form an $S U(n)$-valued $C^{\infty}$ function on $\mathbb{C} P(n-1)$, also denoted as $L^{y}$. The restriction of this matrix function to $\mathbb{C} P(n-1)_{0}$ satisfies the following identity:

$$
\left(L^{y} \circ \mathcal{E}\right)(\xi, \tau)=\Delta(\tau)^{-1} L_{y}^{\mathrm{loc}}\left(\delta(\xi), \rho(\tau)^{-1}\right) \Delta(\tau)
$$

where $\mathcal{E}$ denotes the parametrization introduced in (4.3) and

$$
\Delta(\tau):=\operatorname{diag}\left(\tau_{1}, \ldots, \tau_{n-1}, 1\right) .
$$


Proof. It follows from what we established before that the formulas (4.53) and (4.54) yield $C^{\infty}$ functions on $S_{\chi_{0}}^{2 n-1}$, which are obviously invariant under the $U(1)$-action $u \mapsto e^{\mathrm{i} \gamma} u$. By using (4.5) and (4.56), one readily checks that the right-hand-side of (4.55) is equal to the matrix

$$
\operatorname{diag}\left(\tau_{1}^{-1}, \ldots, \tau_{n-1}^{-1}, 1\right) L_{y}^{\mathrm{loc}}\left(\delta(\xi), \mathbf{1}_{n}\right) \operatorname{diag}\left(1, \tau_{1}, \ldots, \tau_{n-1}\right) .
$$

On account of (4.51) and (4.52), this is further equal to the matrix $L^{y}(u)$ at $u$ given by

$$
u_{i}=\tau_{i} \sqrt{\xi_{i}-|y|} \quad(i=1, \ldots, n-1), \quad u_{n}=\sqrt{\xi_{n}-|y|} .
$$

By the definition of the map $\mathcal{E}(4.3)$, this proves the equality (4.55). Finally, note that $L^{y}$ is $S U(n)$ valued since its restriction to $\mathbb{C} P(n-1)_{0}$ is $S U(n)$ valued.

Remark 7. The $C^{\infty}$ function $L^{y}: \mathbb{C} P(n-1) \rightarrow S U(n)$ specified by Lemma 8 will be referred to as the global Lax matrix. Since by (4.55) it reduces to a conjugate of the local Lax matrix $L_{y}^{\text {loc }}$ on $\mathbb{C} P(n-1)_{0}, L^{y}$ can serve as the Lax matrix of an integrable system defined on $\mathbb{C} P(n-1)$. Apart from slight differences of conventions, our $L^{y}$ actually coincides with the Lax matrix of the compactified $\mathrm{III}_{\mathrm{b}}$ system constructed in [31] (pages 311-312 loc. cit.) relying on arguments similar to the above. It might be worth noting $L^{y}$ is not only $C^{\infty}$ but real-analytic on $\mathbb{C} P(n-1)$, as follows by inspection of the above proof and established also in [31].

\subsection{Construction of the global Delzant map $f_{\beta}$}

Let us remember that $\mu^{-1}\left(\mu_{0}\right)$ is the total space of a principal bundle with projection

$$
\phi_{\beta} \circ p: \mu^{-1}\left(\mu_{0}\right) \rightarrow P \rightarrow \mathbb{C} P(n-1)
$$

We established the characteristic properties (3.78) of $\phi_{\beta}$, but not yet its explicit form. Now we wish to give a construction of the inverse map $f_{\beta}=\phi_{\beta}^{-1}$. Our plan to achieve this is as follows. We first cover $\mathbb{C} P(n-1)$ by $n$ 'coordinate charts' $\mathbb{C} P(n-1)_{j}$, where $\mathbb{C} P(n-1)_{j} \subset \mathbb{C} P(n-1)$ $(j=1, \ldots, n)$ is by definition the set of those $U(1)$ orbits in $S_{\chi_{0}}^{2 n-1}$ (4.45) for which $u_{j} \neq 0$. By an explicit formula, we then introduce a map

$$
F_{j}: \mathbb{C} P(n-1)_{j} \rightarrow \mu^{-1}\left(\mu_{0}\right)
$$

which turns out to define a local section of the principal bundle over $\mathbb{C} P(n-1)_{j}$. These maps have the property that the projected maps

$$
p \circ F_{j}: \mathbb{C} P(n-1)_{j} \rightarrow P
$$

coincide on the overlaps of their domains, and engender the desired global symplectomorphism

$$
f_{\beta}: \mathbb{C} P(n-1) \rightarrow P
$$

We shall also see that $f_{\beta}$ extends the map $f_{0}: \mathbb{C} P(n-1)_{0} \rightarrow P$ described in Theorem 4 . This will be shown by using that $\mathbb{C} P(n-1)_{0}=\cap_{j=1}^{n} \mathbb{C} P(n-1)_{j}$ and $f_{0}$ has the form $f_{0}=p \circ F_{0}$ with the local section $F_{0}: \mathbb{C} P(n-1)_{0} \rightarrow \mu^{-1}\left(\mu_{0}\right)$ given in equation (4.23). 
To begin, we introduce coordinates on $\mathbb{C} P(n-1)_{j}$ for each $j$ by considering the $n$-tuples

$$
u^{j}:=\left(u_{1}^{j}, \ldots, u_{n}^{j}\right)
$$

subject to the conditions

$$
\sum_{k \neq j}^{n}\left|u_{k}^{j}\right|^{2}<\chi_{0}, \quad u_{j}^{j}:=\sqrt{\chi_{0}-\sum_{k \neq j}^{n}\left|u_{k}^{j}\right|^{2}}, \quad \chi_{0}=\pi-n|y| .
$$

Since $u_{j}^{j}$ is a function of the other components of $u^{j}$, we may think of $u^{j}$ as a variable running over the open ball $\mathfrak{B}_{\chi_{0}} \subset \mathbb{C}^{n-1}$ defined by

$$
\mathfrak{B}_{\chi_{0}}:=\left\{\left.\left(z_{1}, \ldots, z_{n-1}\right) \in \mathbb{C}^{n-1}\left|\sum_{k=1}^{n-1}\right| z_{k}\right|^{2}<\chi_{0}\right\} .
$$

Accordingly, we let

$$
\left(\mathbb{C} P(n-1)_{j}, \mathfrak{B}_{\chi_{0}}^{j}\right)
$$

denote the dense open subset $\mathbb{C} P(n-1)_{j}$ of $\mathbb{C} P(n-1)$ equipped with the coordinates $u_{k}^{j}$. For notational convenience, we also keep the component $u_{j}^{j}$, although it is a function of the true coordinates $u_{k}^{j}(k \neq j)$ on $\mathbb{C} P(n-1)_{j}$. In this notation the formula for the change of coordinates between the charts in especially simple. For example, the $n$ alternative coordinates $u^{j}(j=1, \ldots, n)$ of the same point $\mathcal{E}(\xi, \tau) \in \mathbb{C} P(n-1)_{0}(4.3)$ can be written briefly as

$$
u_{k}^{j}=r_{k} \bar{\tau}_{j} \tau_{k} \quad \text { with } \quad r_{k}=\sqrt{\xi_{k}-|y|}, \quad k=1, \ldots, n, \quad \tau_{n}:=1 .
$$

Since it is a reduction of $\Omega_{\mathbb{C}^{n}}$ (3.67), the symplectic form $\chi_{0} \omega_{\mathrm{FS}}$ is represented by the Darboux form i $\sum_{k \neq j} d \bar{u}_{k}^{j} \wedge d u_{k}^{j}$ on the chart (4.66)

Consider now the component $v_{j}$ of the vector (4.6). Notice from Lemma 7 that (since $y>0$ ) $v_{j}(\xi, y)$ yields a $C^{\infty}$ function on $\mathbb{C} P(n-1)_{j}$. Then, for each $j=1, \ldots, n$, define the $U(n)$ valued function $g_{y}^{j}(\xi)$ as follows. First of all, set $g_{y}^{n}:=g_{y}$ in (4.8). For $1 \leq j<n$, let $T^{j}$ denote the $n$ by $n$ transposition matrix given explicitly by

$$
T^{j}:=\mathbf{1}_{n}-E_{j, j}-E_{n, n}+E_{j, n}+E_{n, j} .
$$

Then using the formula (4.7) and the vector $v(\xi, y)$ in (4.6) define

$$
g_{y}^{j}(\xi):=T^{j} g\left(T^{j} v(\xi, y)\right) .
$$

It is clear that $g_{y}^{j}(\xi)$ is actually a real-orthogonal matrix for all $j$. Moreover, we point out that

$$
g_{y}^{j}(\xi)=g_{y}^{n}(\xi) \eta_{y}^{j}(\xi) \quad \text { with } \quad \eta_{y}^{j}(\xi) \in U(n) \quad \text { for which } \quad \eta_{y}^{j}(\xi) \mu_{0} \eta_{y}^{j}(\xi)^{-1}=\mu_{0},
$$

which holds simply because the matrices $g_{y}^{j}(\xi)$ have the same last column for all $j$.

As examples that illustrate well the general case, for $n=3$ we display the matrices

$$
g_{y}^{1}=\left[\begin{array}{ccc}
-v_{3} & -v_{2} & v_{1} \\
-\frac{v_{2} v_{3}}{d_{1}} & \left(1-\frac{v_{2}^{2}}{d_{1}}\right) & v_{2} \\
\left(1-\frac{v_{3}^{2}}{d_{1}}\right) & -\frac{v_{3} v_{2}}{d_{1}} & v_{3}
\end{array}\right] \quad \text { and } \quad g_{y}^{2}=\left[\begin{array}{ccc}
\left(1-\frac{v_{1}^{2}}{d_{2}}\right) & -\frac{v_{1} v_{3}}{d_{2}} & v_{1} \\
-v_{1} & -v_{3} & v_{2} \\
-\frac{v_{3} v_{1}}{d_{2}} & \left(1-\frac{v_{3}^{2}}{d_{2}}\right) & v_{3}
\end{array}\right]
$$


where $d_{1}:=1+v_{1}, d_{2}:=1+v_{2}$ and $v:=v(\xi, y)$ in (4.6). The point is that, in general, $g_{y}^{j}$ contains the denominator $d_{j}=1+v_{j}$, which yields a $C^{\infty}$ function on $\mathbb{C} P(n-1)_{j}$.

The rationale behind the definition of $g_{y}^{j}$ is that using $\Delta_{n}:=\Delta$ in (4.56) and introducing

$$
\Delta_{j}(\tau):=T^{j} \Delta_{n}(\tau) T^{j}=\operatorname{diag}\left(\tau_{1}, \ldots, 1, \ldots, \tau_{n-1}, \tau_{j}\right), \quad \forall j=1, \ldots, n-1,
$$

where the entry 1 appears in the $j j$ position, one can verify the following lemma.

Lemma 9. The $U(n)$-valued $C^{\infty}$ function $\mathcal{G}_{y}^{j}$ defined on $\mathbb{C} P(n-1)_{0}$ by the formula

$$
\left(\mathcal{G}_{y}^{j} \circ \mathcal{E}\right)(\xi, \tau):=\Delta(\tau)^{-1} g_{y}^{j}(\xi) \Delta_{j}(\tau), \quad j=1, \ldots, n,
$$

extends to a $C^{\infty}$ function on $\mathbb{C} P(n-1){ }_{j}$. The extended function is denoted by the same letter, $\mathcal{G}_{y}^{j}: \mathbb{C} P(n-1)_{j} \rightarrow U(n)$.

Proof. This is a simple inspection of the matrix elements of $\mathcal{G}_{y}^{j}$ based on the properties of $w_{j}^{y}(\xi)$ in (4.47) and the formula (4.67). Indeed, if $u^{j}$ is representative of $\mathcal{E}(\xi, \tau)$ according to (4.67), then for $j \neq n$ one finds for example that

$\mathcal{G}_{y}^{j}\left(u^{j}\right)_{k n} \sim \bar{u}_{k}^{j}, \quad \mathcal{G}_{y}^{j}\left(u^{j}\right)_{n j} \sim 1, \quad \mathcal{G}_{y}^{j}\left(u^{j}\right)_{j j} \sim u_{n}^{j}, \quad \mathcal{G}_{y}^{j}\left(u^{j}\right)_{k l} \sim \delta_{k l}+\left(1-\delta_{k l}\right) \bar{u}_{k}^{j} u_{l}^{j}$ for $k, l \notin\{j, n\}$,

where the symbol $\sim$ means proportionality by a function of $\xi$ that extends to a $C^{\infty}$, nowhere zero function on $\mathbb{C} P(n-1)_{j}$. The relations (4.74), and similar relations that hold for all matrix elements of $\mathcal{G}_{y}^{j}\left(u^{j}\right)$, including $j=n$, imply that $\mathcal{G}_{y}^{j}$ extends from $\mathbb{C} P(n-1)_{0}$ to a $C^{\infty}$ function on $\mathbb{C} P(n-1)_{j}$.

For any fixed $j=1, \ldots, n$ and $y>0$, define the $S U(n) \times S U(n)$ valued $C^{\infty}$ map $F_{j}$ on the chart $\left(\mathbb{C} P(n-1)_{j}, \mathfrak{B}_{\chi_{0}}^{j}\right)$ by the following formula:

$$
F_{j}\left(u^{j}\right):=\left(\mathcal{G}_{y}^{j}\left(u^{j}\right)^{-1} L^{y}\left(u^{j}\right) \mathcal{G}_{y}^{j}\left(u^{j}\right), \mathcal{G}_{y}^{j}\left(u^{j}\right)^{-1} \delta(\xi) \mathcal{G}_{y}^{j}\left(u^{j}\right)\right)
$$

where $\xi_{i}=\left|u_{i}^{j}\right|^{2}+y$ for every $i=1, \ldots, n$ and $L^{y}$ is the global Lax matrix given in Lemma 8 .

Theorem 5. The maps $F_{j}$ (4.75) enjoy the following properties:

1. $F_{j}\left(u^{j}\right)$ belongs to $\mu^{-1}\left(\mu_{0}\right)$ and $p \circ F_{j}: \mathbb{C} P(n-1)_{j} \rightarrow P$ is a smooth map.

2. $p \circ F_{j}$ coincides with $p \circ F_{k}$ on $\mathbb{C} P(n-1)_{j} \cap \mathbb{C} P(n-1)_{k}$ and it coincides with $f_{0}$ of Theorem 4 on $\mathbb{C} P(n-1)_{0}=\cap_{j=1}^{n} \mathbb{C} P(n-1)_{j}$.

3. One can define a smooth map $f_{\beta}: \mathbb{C} P(n-1) \rightarrow P$ by requiring that $f_{\beta}$ coincides with $p \circ F_{j}$ on $\mathbb{C} P(n-1)_{j}$. The so-obtained map satisfies

$$
f_{\beta}^{*}(\hat{\omega})=\chi_{0} \omega_{\mathrm{FS}} \quad \text { and } \quad f_{\beta}^{*}(\hat{\beta})=\mathcal{J} .
$$

4. The $\operatorname{map} f_{\beta}$ is surjective and injective. 
Consequently, $f_{\beta}$ is a symplectomorphism that extends the local Delzant map $f_{0}$ of Theorem 4 and its inverse $\phi_{\beta}:=f_{\beta}^{-1}$ is a Delzant symplectomorphism satisfying equation (3.78).

Proof. It follows directly from the definitions that

$$
\left(F_{j} \circ \mathcal{E}\right)(\xi, \tau)=\Psi_{\eta_{y}^{j}(\xi, \tau)^{-1}}\left(\left(F_{0} \circ \mathcal{E}\right)(\xi, \tau)\right), \quad \forall(\xi, \tau) \in \mathcal{P}_{y}^{0} \times \mathbb{T}_{n-1},
$$

where we use (4.23) and the definition $\eta_{y}^{j}(\xi, \tau):=\eta_{y}^{j}(\xi) \Delta_{j}(\tau)$ with (4.70). Since $\eta_{y}^{j}(\xi, \tau)$ belongs to the little group of $\mu_{0}$ in $U(n)$, this entails that $\left(F_{j} \circ \mathcal{E}\right)(\xi, \tau) \in \mu^{-1}\left(\mu_{0}\right)$. Then we obtain property 1 since $\mu^{-1}\left(\mu_{0}\right) \subset D$ is closed, the values $\mathcal{E}(\xi, \tau)$ cover $\mathbb{C} P(n-1)_{0}$ which is dense in $\mathbb{C} P(n-1)_{j}$, and $F_{j}: \mathbb{C} P(n-1)_{j} \rightarrow D$ is a $C^{\infty}$ map as shown by its formula (4.75).

Property 2 holds since $\mathbb{C} P(n-1)_{0} \subset \mathbb{C} P(n-1)_{j} \cap \mathbb{C} P(n-1)_{k}$ is dense, and $p \circ F_{j}$ coincides with $f_{0}=p \circ F_{0}$ on $\mathbb{C} P(n-1)_{0}$ because of (4.77).

Property 3 is immediate from the preceding properties and the fact that $f_{0}$ is a local Delzant map satisfying (4.10) and (4.11).

To establish the surjectivity of $f_{\beta}$, notice that equivariance with respect to the torus actions (3.74) and (3.54),

$$
f_{\beta} \circ \mathcal{R}_{\tau}=\hat{\Psi}_{\tau}^{b} \circ f_{\beta}, \quad \forall \tau \in \mathbb{T}_{n-1},
$$

follows from (4.76) . Then $f_{\beta}^{*}(\hat{\beta})=\mathcal{J}$ and (4.78) imply that the image of $f_{\beta}$ contains each $\mathbb{T}_{n-1}$-orbit in $P$. This entails the surjectivity.

Our final task it to demonstrate the injectivity of $f_{\beta}$. To do this, we remark that if $f_{\beta}$ takes the same values on two elements of $\mathbb{C} P(n-1)$, then those elements must belong to the same chart $\mathbb{C} P(n-1)_{j}$ at least for one $j$. Indeed, this is a consequence of the second equality in (4.76) and the definition of $\mathbb{C} P(n-1)_{j}$. Then, assume that $f_{\beta}\left(u^{j}\right)=f_{\beta}\left(z^{j}\right)$ for two elements $u^{j}, z^{j} \in \mathfrak{B}_{\chi_{0}}^{j}$. By the definition of $f_{\beta}$, this is equivalent to the existence of an element $\eta$ from the little group of $\mu_{0}$ in $U(n)$ such that

$$
\begin{array}{r}
\left(\eta^{-1} \mathcal{G}_{y}^{j}\left(u^{j}\right)^{-1} L^{y}\left(u^{j}\right) \mathcal{G}_{y}^{j}\left(u^{j}\right) \eta, \eta^{-1} \mathcal{G}_{y}^{j}\left(u^{j}\right)^{-1} \delta(\xi) \mathcal{G}_{y}^{j}\left(u^{j}\right) \eta\right)= \\
=\left(\mathcal{G}_{y}^{j}\left(z^{j}\right)^{-1} L^{y}\left(z^{j}\right) \mathcal{G}_{y}^{j}\left(z^{j}\right), \mathcal{G}_{y}^{j}\left(z^{j}\right)^{-1} \delta(\xi) \mathcal{G}_{y}^{j}\left(z^{j}\right)\right)
\end{array}
$$

where $\xi$ is given by $\xi_{i}=\left|u_{i}^{j}\right|^{2}+|y|=\left|z_{i}^{j}\right|^{2}+|y|$. We see from the second component of (4.79) that

$$
T:=\mathcal{G}_{y}^{j}\left(u^{j}\right) \eta \mathcal{G}_{y}^{j}\left(z^{j}\right)^{-1}
$$

must belong to the torus $\mathbb{T}_{n} \subset U(n)$. Then the first component of (4.79) and the fact that $L_{k, k+1}^{y}$ (4.54) depends only on $\xi$ and never vanishes for any $k$ imply that $T=\lambda \mathbf{1}_{n}$ for some $\lambda \in U(1)$. Upon re-substitution into the first component of (4.79), this gives the equality

$$
L^{y}\left(u^{j}\right)=L^{y}\left(z^{j}\right) .
$$

By using that the components of $\Lambda^{y}(\xi)$ in (4.53) are non-zero, we infer from the inspection of $L_{k, l}^{y}\left(u^{j}\right)=L_{k, l}^{y}\left(z^{j}\right)$ for the fixed index $l=j+1(l:=1$ if $j=n)$ that $\bar{u}_{k}^{j} u_{j}^{j}=\bar{z}_{k}^{j} z_{j}^{j}$ holds for each $k$. Since $u_{j}^{j}=z_{j}^{j}$ as this component depends only on $\xi$, and $u_{j}^{j} \neq 0$, we conclude that $z^{j}=u^{j}$, whereby the injectivity of $f_{\beta}$ follows. 
As an alternative to the above self-contained reasoning, we can also give a shorter proof of the injectivity of $f_{\beta}$ by invoking that for any Hamiltonian toric manifold and the pre-image of any moment map value there exists a certain subtorus that acts freely on that pre-image [2]. The subtorus in question (which is the whole torus for the interior of the Delzant polytope) depends only on the moment map value, and by using this the injectivity of $f_{\beta}$ follows easily from $f_{\beta}^{*}(\hat{\beta})=\mathcal{J}$ and (4.78).

Remark 8. It is readily seen from the above that the maps $F_{j}: \mathbb{C} P(n-1)_{j} \rightarrow \mu^{-1}\left(\mu_{0}\right)$ defined by (4.75) are indeed local sections of the principal bundle in (4.59) whose base is $\mathbb{C} P(n-1)$ and total space is the constraint surface $\mu^{-1}\left(\mu_{0}\right)$. We constructed the global Delzant symplectomorphism $f_{\beta}: \mathbb{C} P(n-1) \rightarrow P$ by patching together the projected maps $p \circ F_{j}$.

\subsection{The global Delzant map $f_{\alpha}$ and involution properties}

We here present the construction of $f_{\alpha}$ in terms of $f_{\beta}$, and establish the involution properties (4.92) and (4.118) for the maps (4.117) whose significance will become clear in Section 5.

We need some preparations. First, let us define the map $\nu$ on the unreduced double by

$$
\nu(A, B):=(\bar{B}, \bar{A}),
$$

where 'bar' means complex conjugation. This is an involution of $D$ that enjoys the properties

$$
\nu^{*}(\omega)=-\omega, \quad \nu^{*}(\mu)=\bar{\mu}^{-1}, \quad \nu \circ \Psi_{g}=\Psi_{\bar{g}} \circ \nu \quad(\forall g \in G) .
$$

By using these properties and the fact that $\left(\bar{\mu}_{0}\right)^{-1}=\mu_{0}$, we see that $\nu$ maps $\mu^{-1}\left(\mu_{0}\right)$ to itself and it induces an anti-symplectic involution, $\hat{\nu}$, of the reduced phase space $P$.

Second, let us define the anti-symplectic involution $\Gamma$ of the symplectic vector space $\mathbb{C}^{n}$ by

$$
\Gamma\left(u_{1}, \ldots, u_{n-1}, u_{n}\right):=\left(\bar{u}_{n-1}, \ldots, \bar{u}_{1}, \bar{u}_{n}\right),
$$

which acts as 'reflection composed with complex conjugation' on the first $(n-1)$ coordinates. Straightforwardly, $\Gamma$ induces an anti-symplectic involution, $\hat{\Gamma}$, of $\left(\mathbb{C} P(n-1), \chi_{0} \omega_{\mathrm{FS}}\right)$. We also need the anti-symplectic involution $\hat{C}: \mathbb{C} P(n-1) \rightarrow \mathbb{C} P(n-1)$ that descends from componentwise complex conjugation on $\mathbb{C}^{n}$, i.e. for which

$$
\hat{C} \circ \pi_{\chi_{0}}\left(u_{1}, \ldots, u_{n-1}, u_{n}\right)=\pi_{\chi_{0}}\left(\bar{u}_{1}, \ldots, \bar{u}_{n-1}, \bar{u}_{n}\right) \quad \text { with } \quad u \in S_{\chi_{0}}^{2 n-1},
$$

as well the symplectic involution $\hat{\sigma}$ furnished by 8

$$
\hat{\sigma}:=\hat{C} \circ \hat{\Gamma}=\hat{\Gamma} \circ \hat{C} .
$$

Third, note that the spectral functions $\Xi_{k}$ defined in Section 2 (Eq. (2.22)) verify the identity

$$
\Xi_{k}(\bar{A})=\Xi_{n-k}(A) \quad \forall k=1, \ldots, n-1, \quad \Xi_{n}(\bar{A})=\Xi_{n}(A), \quad \forall A \in G .
$$

\footnotetext{
${ }^{8}$ The value $n=2$ is special since in this case $\hat{C}=\hat{\Gamma}$ and $\hat{\sigma}$ (as well as $\sigma$ in (4.88) ) becomes the identity map.
} 
This can be checked by direct calculation starting from (2.19), and also follows from well-known group theoretic facts via the formula (2.20).

Fourth, let $\sigma$ denote the involutive map on $\mathbb{C}^{n-1}$ given by

$$
\sigma(x)_{k}:=x_{n-k}, \quad \forall x \in \mathbb{C}^{n-1}, \quad \forall k=1, \ldots, n-1 .
$$

As a result of (4.87), the $\mathbb{R}^{n-1}$-valued spectral Hamiltonians, and their respective reductions, are subject to the relations

$$
\alpha=\sigma \circ \beta \circ \nu, \quad \hat{\alpha}=\sigma \circ \hat{\beta} \circ \hat{\nu} .
$$

Fifth, the moment map $\mathcal{J}: \mathbb{C} P(n-1) \rightarrow \mathbb{R}^{n-1}$ of the rotational $\mathbb{T}_{n-1}$-action used in Theorem 3 obeys

$$
\mathcal{J} \circ \hat{\Gamma}=\mathcal{J} \circ \hat{\sigma}=\sigma \circ \mathcal{J}
$$

Now we can construct $f_{\alpha}$ in terms of $f_{\beta}$.

Theorem 6. If $f_{\beta}$ is a Delzant map in the sense of Theorem 3 (cf. also Eq. (3.79)), then

$$
f_{\alpha}:=\hat{\nu} \circ f_{\beta} \circ \hat{\Gamma}
$$

is also a Delzant map in the sense of Theorem 3. Equation 4.91) implies the involution property

$$
\left(\hat{\Gamma} \circ f_{\alpha}^{-1} \circ f_{\beta}\right)^{2}=\operatorname{id}_{\mathbb{C} P(n-1)} .
$$

Proof. It is clear that $f_{\alpha}$ as defined by (4.91) is a symplectomorphism provided that $f_{\beta}$ is a symplectomorphism. By assuming that $\hat{\beta} \circ f_{\beta}=\mathcal{J}$, (4.91) and the previous relations entail

$$
\hat{\alpha} \circ f_{\alpha}=\hat{\alpha} \circ \hat{\nu} \circ f_{\beta} \circ \hat{\Gamma}=\sigma \circ \hat{\beta} \circ f_{\beta} \circ \hat{\Gamma}=\sigma \circ \mathcal{J} \circ \hat{\Gamma}=\mathcal{J}
$$

which is the required property of the Delzant map $f_{\alpha}$. Eq. (4.92) follows directly from (4.91).

It is worth noting that we did not use the explicit formula of $f_{\beta}$ to establish Theorem 6 .

Lemma 10. It follows from the local formula (4.9) of $f_{\beta}$ that the restriction of the map $f_{\alpha}$ (4.91) to $\mathbb{C} P(n-1)_{0}$ operates according to

$$
\left(f_{\alpha} \circ \mathcal{E}\right)(\xi, \tau)=\left(p \circ \Psi_{g_{-y}(\xi)^{-1}}\right)\left(\delta(\xi), L_{-y}^{\text {loc }}(\delta(\xi), \rho(\tau))\right),
$$

using the same notations as in (4.9) and $\Psi$ in (2.10). Moreover, the following identity holds:

$$
f_{\beta}^{-1} \circ f_{\alpha}=\hat{\Gamma} \circ\left(f_{\beta}^{-1} \circ f_{\alpha}\right) \circ \hat{C} .
$$


Proof. For typographic reasons, in this proof we use both alternative notations

$$
M^{*} \equiv \bar{M}
$$

to denote the complex conjugate of any matrix $M ; M^{t}$ denotes transpose and $M^{\dagger}$ adjoint. We begin the proof of formula (4.94) by remarking that the map $\mathcal{E}(\underline{4.3}$ ) satisfies

$$
(\hat{\Gamma} \circ \mathcal{E})(\xi, \tau)=\mathcal{E}(\sigma(\xi), \sigma(\bar{\tau})), \quad \forall(\xi, \tau) \in \mathcal{P}_{y}^{0} \times \mathbb{T}_{n-1}
$$

with $\hat{\Gamma}$ and $\sigma$ defined earlier. Then the combination of equations (4.9), (4.91) and (4.97) gives

$$
\left(f_{\alpha} \circ \mathcal{E}\right)(\xi, \tau)=p \circ \Psi_{g_{y}(\sigma(\xi))^{-1}}\left(\delta(\sigma(\xi))^{*}, L_{y}^{\text {loc }}\left(\delta(\sigma(\xi)), \rho(\sigma(\bar{\tau}))^{-1}\right)^{*}\right) .
$$

Here, we have taken into account that $g_{y}(\sigma(\xi))^{*}=g_{y}(\sigma(\xi))$ holds since $g_{y}(4.7)$ is real. Let $\eta_{0}$ be the $n \times n$ matrix whose non-zero entries are $\left(\eta_{0}\right)_{j, n+1-j}=1$ for all $j=1, \ldots, n ; \eta_{0}=\eta_{0}^{-1}=\eta_{0}^{t}$. It is not difficult, although somewhat long, to check that

$$
\delta(\sigma(\xi))^{*}=\eta_{0} \delta(\xi) \eta_{0}
$$

and

$$
L_{y}^{\text {loc }}\left(\delta(\sigma(\xi)), \rho(\sigma(\bar{\tau}))^{-1}\right)^{*}=\eta_{0} L_{-y}^{\text {loc }}(\delta(\xi), \rho(\tau)) \eta_{0}
$$

In the course of deriving these relations we utilized that

$$
W_{j}(\delta(\sigma(\xi)), y)=W_{n+1-j}(\xi,-y), \quad \forall j=1, \ldots, n,
$$

and

$$
\rho(\sigma(\bar{\tau}))^{-1}=\rho(\sigma(\tau))=\eta_{0} \rho(\tau)^{-1} \eta_{0} .
$$

By using (4.99) and (4.100), we can rewrite (4.98) as

$$
\left(f_{\alpha} \circ \mathcal{E}\right)(\xi, \tau)=p \circ \Psi_{\left(\eta_{0} g_{y}(\sigma(\xi))\right)^{-1}}\left(\delta(\xi), L_{-y}^{\text {loc }}(\delta(\xi), \rho(\tau))\right) .
$$

It follows from (4.101) that $\eta_{0} g_{y}(\sigma(\xi))$ is a unitary matrix whose last column is given by the vector $v(\xi,-y)$ defined in (4.6). This permits to conclude that $\eta_{0} g_{y}(\sigma(\xi))=g_{-y}(\xi) g$ with some ( $\xi$ and $y$-dependent) $g \in U(n)$ for which $g \mu_{0} g^{-1}=\mu_{0}$. Taking into account that $\Psi_{g}$ is a gauge transformation, equation (4.103) implies the desired formula (4.94).

Now are are going to prove the identity (4.95). It is enough to verify this identity on the dense open submanifold of $\mathbb{C} P(n-1)_{0}$ whose image under the map $f_{\beta}^{-1} \circ f_{\alpha}$ is also contained in $\mathbb{C} P(n-1)_{0}$. For any $\mathcal{E}(\xi, \tau)$ from this submanifold, we define $\left(\xi^{\prime}, \tau^{\prime}\right) \in \mathcal{P}_{y}^{0} \times \mathbb{T}_{n-1}$ by the equation

$$
\left(f_{\beta}^{-1} \circ f_{\alpha}\right)(\mathcal{E}(\xi, \tau))=\mathcal{E}\left(\xi^{\prime}, \tau^{\prime}\right)
$$

which can be rewritten equivalently as

$$
f_{\alpha}(\mathcal{E}(\xi, \tau))=f_{\beta}\left(\mathcal{E}\left(\xi^{\prime}, \tau^{\prime}\right)\right)
$$

Then the claim (4.95) can be reformulated as the statement that the relation (4.105) is equivalent to the relation

$$
\left(\hat{\Gamma} \circ\left(f_{\beta}^{-1} \circ f_{\alpha}\right) \circ \hat{C}\right)(\mathcal{E}(\xi, \tau))=\mathcal{E}\left(\xi^{\prime}, \tau^{\prime}\right)
$$


which (by taking into account (4.97) ) is in turn equivalent to

$$
f_{\alpha}(\mathcal{E}(\xi, \bar{\tau}))=f_{\beta}\left(\mathcal{E}\left(\sigma\left(\xi^{\prime}\right), \sigma\left(\tau^{\prime}\right)^{-1}\right)\right) .
$$

Consequently, we have to show that (4.105) is equivalent to (4.107).

We now introduce the notation $\left(A_{1}, B_{1}\right) \sim\left(A_{2}, B_{2}\right)$ for elements of the double $D$ for which there exists $g \in U(n)$ such that $g A_{1} g^{-1}=A_{2}$ and $g B_{1} g^{-1}=B_{2}$. We notice that two pairs $\left(A_{1}, B_{1}\right)$ and $\left(A_{2}, B_{2}\right)$ in $\mu^{-1}\left(\mu_{0}\right)$ represent the same element of $P$ if and only if $\left(A_{1}, B_{1}\right) \sim$ $\left(A_{2}, B_{2}\right)$. Therefore, by using the local formulae (4.9) of $f_{\beta}$ and (4.94) of $f_{\alpha}$, we can reformulate the equivalence of (4.105) and (4.107) as the equivalence between the relation

$$
\left(\delta(\xi), L_{-y}^{\mathrm{loc}}(\delta(\xi), \rho(\tau))\right) \sim\left(L_{y}^{\mathrm{loc}}\left(\delta\left(\xi^{\prime}\right), \rho\left(\tau^{\prime}\right)^{-1}\right), \delta\left(\xi^{\prime}\right)\right)
$$

and the relation

$$
\left(\delta(\xi), L_{-y}^{\mathrm{loc}}(\delta(\xi), \rho(\bar{\tau}))\right) \sim\left(L_{y}^{\mathrm{loc}}\left(\delta\left(\sigma\left(\xi^{\prime}\right)\right), \rho\left(\sigma\left(\tau^{\prime}\right)\right)\right), \delta\left(\sigma\left(\xi^{\prime}\right)\right)\right) .
$$

By applying (4.99) and (4.100), we observe that (4.109) is equivalent to

$$
\left(\delta(\xi), L_{-y}^{\mathrm{loc}}(\delta(\xi), \rho(\bar{\tau}))\right) \sim\left(L_{-y}^{\mathrm{loc}}\left(\delta\left(\xi^{\prime}\right), \rho\left(\tau^{\prime}\right)\right)^{*}, \delta\left(\xi^{\prime}\right)^{*}\right) .
$$

To finish the proof, we need the identities

$$
\begin{gathered}
L_{-y}^{\mathrm{loc}}\left(\delta\left(\xi^{\prime}\right), \rho\left(\tau^{\prime}\right)\right)^{*}=\rho\left(\tau^{\prime}\right)\left[L_{y}^{\mathrm{loc}}\left(\delta\left(\xi^{\prime}\right), \rho\left(\tau^{\prime}\right)^{-1}\right)\right]^{t} \rho\left(\tau^{\prime}\right)^{-1}, \\
L_{-y}^{\mathrm{loc}}(\delta(\xi), \rho(\tau))^{*}=\delta(\xi) L_{-y}^{\mathrm{loc}}\left(\delta(\xi), \rho(\tau)^{-1}\right) \delta(\xi)^{-1},
\end{gathered}
$$

which can be readily verified.

Now suppose that (4.108) holds (for some arbitrarily fixed $(\xi, \tau)$ ). This assumption is equivalent to the existence of a unitary matrix $g$ for which

$$
g \delta(\xi) g^{-1}=L_{y}^{\mathrm{loc}}\left(\delta\left(\xi^{\prime}\right), \rho\left(\tau^{\prime}\right)^{-1}\right) \quad \text { and } \quad g^{-1} \delta\left(\xi^{\prime}\right) g=L_{-y}^{\mathrm{loc}}(\delta(\xi), \rho(\tau)) .
$$

Then, by means of (4.111), the validity of the first equality in (4.113) implies that

$$
L_{-y}^{\mathrm{loc}}\left(\delta\left(\xi^{\prime}\right), \rho\left(\tau^{\prime}\right)\right)^{*}=\rho\left(\tau^{\prime}\right)\left(g \delta(\xi) g^{-1}\right)^{t} \rho\left(\tau^{\prime}\right)^{-1}=\left[\rho\left(\tau^{\prime}\right) \bar{g} \delta(\xi)\right] \delta(\xi)\left[\rho\left(\tau^{\prime}\right) \bar{g} \delta(\xi)\right]^{-1},
$$

which can be recognized as the 'first component' of the relation (4.110). By using (4.112), the second equality in (4.113) becomes

$$
\delta\left(\xi^{\prime}\right)^{*}=\bar{g} L_{-y}^{\mathrm{loc}}(\delta(\xi), \rho(\tau))^{*} \bar{g}^{-1}=\bar{g} \delta(\xi) L_{-y}^{\mathrm{loc}}\left(\delta(\xi), \rho(\tau)^{-1}\right)(\bar{g} \delta(\xi))^{-1},
$$

which (since $\delta$ and $\rho$ take values in $\mathbb{T}_{n}$ ) entails that

$$
\delta\left(\xi^{\prime}\right)^{*}=\left[\rho\left(\tau^{\prime}\right) \bar{g} \delta(\xi)\right] L_{-y}^{\mathrm{loc}}\left(\delta(\xi), \rho(\tau)^{-1}\right)\left[\rho\left(\tau^{\prime}\right) \bar{g} \delta(\xi)\right]^{-1} .
$$

Thus we have derived (4.114) and (4.116) from (4.113), which tells us that (4.108) implies (4.109). The converse implication can be demonstrated by following the above equations in reverse order, whereby the proof is complete. 
In Section 5, we shall identify the maps

$$
\mathfrak{S}:=f_{\alpha}^{-1} \circ f_{\beta} \text { and } \mathfrak{R}:=\hat{C} \circ \mathfrak{S}
$$

as the symplectic and respectively the anti-symplectic version of Ruijsenaars' self-duality map of the compactified $\mathrm{III}_{\mathrm{b}}$ system. Then the properties (4.92) and (4.95) will reproduce certain relations established in [31]. The same is true regarding the following identities that can be derived easily from the above:

$$
\mathfrak{S}^{2}=\hat{\sigma} \quad \text { and } \quad \mathfrak{R}^{2}=\operatorname{id}_{\mathbb{C} P(n-1)} .
$$

As for their derivation, the first identity in (4.118) is obtained by recasting (4.92) as

$$
\operatorname{id}_{\mathbb{C} P(n-1)}=\hat{\Gamma} \circ \mathfrak{S} \circ \hat{\Gamma} \circ \mathfrak{S}=\hat{\Gamma} \circ \hat{C} \circ \mathfrak{S}^{2}=\hat{\sigma} \circ \mathfrak{S}^{2},
$$

where we applied (4.95) to establish the second equality. Similarly, we can write

$$
\mathfrak{R}=\hat{C} \circ \mathfrak{S}=\mathfrak{S} \circ \hat{\Gamma}=\hat{\Gamma} \circ(\hat{\Gamma} \circ \mathfrak{S}) \circ \hat{\Gamma}
$$

whereby the second identity in (4.118) follows from (4.119).

Finally, let us record also the following useful identities:

$$
\begin{gathered}
\delta \circ \mathcal{J} \circ \hat{C}=\delta \circ \mathcal{J}, \quad \delta \circ \mathcal{J} \circ \hat{\Gamma}=\delta \circ \mathcal{J} \circ \hat{\sigma}=\eta_{0}(\delta \circ \mathcal{J})^{\dagger} \eta_{0}, \\
L^{y} \circ \hat{C}=\bar{L}^{y}, \quad L^{y} \circ \hat{\Gamma}=\eta_{0}\left(L^{y}\right)^{t} \eta_{0}, \quad L^{y} \circ \hat{\sigma}=\eta_{0}\left(L^{y}\right)^{\dagger} \eta_{0},
\end{gathered}
$$

with $\eta_{0}$ defined above (4.99). The identities in (4.121) are equivalent to (4.90) and (4.99), and those in (4.122) can be verified directly by using the definition of the global Lax matrix $L^{y}$.

\section{Self-duality of the compactified $\mathrm{III}_{\mathrm{b}}$ system}

The construction of the compactified $\mathrm{III}_{\mathrm{b}}$ system and the discovery of its self-duality properties are due to Ruijsenaars [31. Below we first recall the definition of this system from [31], interlaced with some explanatory comments in terms of our present work. Then we establish that our reduction yields the compactified $\mathrm{III}_{\mathrm{b}}$ system, which is the message of Theorem 7 . The subsequent Theorem 8 reproduces the symplectic version of Ruijsenaars' self-duality map as an automatic consequence of our construction. Finally, we briefly explain how the anti-symplectic self-duality involution of Ruijsenaars fits into our framework.

Theorems 7 and 8 represent the first main result of this paper (the second will be encapsulated in Theorem 9 of Section 6). We can be relatively brief here, since these theorems are just easy corollaries of our preceding technical results. In particular, they follow from our detailed description of the Delzant symplectomorphisms $f_{\beta}$ and $f_{\alpha}$ that incorporate the Lax matrix (1.4) and its global extension (4.55). Although we gained inspiration from the seminal paper [31] with which we must compare our results, we wish to emphasize that our approach is self-contained.

The space of particle-positions $\mathcal{D}_{y} \subset S \mathbb{T}_{n}$ of the local $\mathrm{III}_{\mathrm{b}}$ system can be identified with the open polytope $\mathcal{P}_{y}^{0}$ by means of the map $\delta$ (cf. (2.20) $)$. Although the true particle-positions 
are the components of $\delta(\xi)$, we may (and often do) regard the equivalent $\xi \in \mathcal{P}_{y}^{0}$ as the local particle-position variable of the system. The canonical conjugates of the coordinates $\xi_{k}$ are the $\theta_{k}$ that parametrize $\Theta=\rho\left(e^{\mathrm{i} \theta}\right)^{-1}$ by (4.5). By inserting these parametrizations into (1.3) one indeed obtains

$$
\Omega^{\mathrm{loc}}=-\frac{1}{2} \operatorname{tr}\left(\delta(\xi)^{-1} d \delta(\xi) \wedge \rho(\tau)^{-1} d \rho(\tau)\right)=\mathrm{i} \sum_{k=1}^{n-1} d \xi_{k} \wedge \tau_{k}^{-1} d \tau_{k}=\sum_{k=1}^{n-1} d \theta_{k} \wedge d \xi_{k} .
$$

Taking $\xi$ and $\tau=e^{\mathrm{i} \theta}$ as the basic variables, from now on we identify the local phase space as

$$
M_{y}^{\mathrm{loc}} \equiv \mathcal{P}_{y}^{0} \times \mathbb{T}_{n-1}=\{(\xi, \tau)\}
$$

The same variables were used in 31 to describe the relative motion of the particles governed by the Hamiltonian (1.1). The local $\mathrm{III}_{\mathrm{b}}$ system of [31] is thus encapsulated by the triple

$$
\left(M_{y}^{\text {loc }}, \Omega^{\text {loc }}, \mathcal{L}_{y}^{\text {loc }}\right)
$$

where the value of the Lax matrix $\mathcal{L}_{y}^{\text {loc }}$ at $(\xi, \tau) \in M_{y}^{\text {loc }}$ is defined to be

$$
\mathcal{L}_{y}^{\mathrm{loc}}(\xi, \tau):=L_{y}^{\mathrm{loc}}\left(\delta(\xi), \rho(\tau)^{-1}\right)
$$

with the expression (1.4). The commuting Hamiltonians of the system are provided by the functions $h \circ \mathcal{L}_{y}^{\text {loc }}$ for all $h \in C^{\infty}\left(G_{\text {reg }}\right)^{G}$. The composed function $h \circ \mathcal{L}_{y}^{\text {loc }}$ belongs to $C^{\infty}\left(M_{y}^{\text {loc }}\right)$ since $\mathcal{L}_{y}^{\text {loc }}: M_{y}^{\text {loc }} \rightarrow G_{\text {reg }}$, as was shown in [31] and follows also from our Theorem 2 combined with Lemma 6.

Expressed in our notations, the compactified $\mathrm{III}_{\mathrm{b}}$ system was defined in [31] as the triple

$$
\left(\mathbb{C} P(n-1), \chi_{0} \omega_{\mathrm{FS}}, L^{y}\right), \quad \chi_{0}=\pi-n|y|,
$$

where $L^{y}$ is the global Lax matrix described in Subsection 4.2. The commuting Hamiltonians of this system were identified in [31] as the $C^{\infty}$ functions 9 of the form $h \circ L^{y}$ for all $h \in C^{\infty}\left(G_{\mathrm{reg}}\right)^{G}$. In particular, it was established in [31] (and is obvious in our setting) that the functions

$$
\Xi_{k} \circ L^{y}, \quad k=1, \ldots, n-1,
$$

represent action-variables for the system (5.5), where $\Xi_{k} \in C^{\infty}\left(G_{\mathrm{reg}}\right)^{G}$ was defined in (2.22). The crucial fact [31] is that by the identification (5.2) the map $\mathcal{E}$ (4.2) embeds the local III $_{\mathrm{b}}$ system into $\mathbb{C} P(n-1)$, converting $M_{y}^{\text {loc }}$ into the dense open submanifold $\mathbb{C} P(n-1)_{0}$. As seen upon comparison of (4.4) and (5.1), this embedding is symplectic. Because of Lemma 8, the commuting Hamiltonians of the local system extend to those of the compactified one. The compactified system has complete flows, simply since $\mathbb{C} P(n-1)$ is compact and the Hamiltonians of interest are smooth.

Theorem 7. For any $h \in C^{\infty}\left(G_{\mathrm{reg}}\right)^{G}$, let $\hat{h}_{1}$ and $\hat{h}_{2}$ denote the reduced Hamiltonians defined by means of equation (2.7) with (2.14). Consider the Delzant symplectomorphisms $f_{\beta}$ and $f_{\alpha}$

\footnotetext{
${ }^{9}$ Actually in 31 (Eq. (5.50) loc. cit.) real-analytic Hamiltonians were studied, which is a negligible difference.
} 
that map $\left(\mathbb{C} P(n-1), \chi_{0} \omega_{\mathrm{FS}}\right)$ to the reduced phase space $(P, \hat{\omega})$ as given by Theorems 5 and 6 . Then, using the preceding notations, the following relations hold for $f_{\beta}$ :

$$
\hat{h}_{1} \circ f_{\beta}=h \circ L^{y}, \quad \hat{h}_{2} \circ f_{\beta}=h \circ \delta \circ \mathcal{J} .
$$

In particular, with $h=\Xi_{k}$ for any $k=1, \ldots, n-1$,

$$
\hat{\alpha}_{k} \circ f_{\beta}=\Xi_{k} \circ L^{y}, \quad \hat{\beta}_{k} \circ f_{\beta}=\mathcal{J}_{k} .
$$

Regarding $f_{\alpha}$, there hold the analogous relations,

$$
\hat{h}_{1} \circ f_{\alpha}=h \circ \delta \circ \mathcal{J}, \quad \hat{h}_{2} \circ f_{\alpha}=h \circ\left(L^{y}\right)^{\dagger},
$$

and in particular

$$
\hat{\alpha}_{k} \circ f_{\alpha}=\mathcal{J}_{k}, \quad \hat{\beta}_{k} \circ f_{\alpha}=\Xi_{n-k} \circ L^{y} .
$$

Proof. After recalling the underlying definitions, all these relations follow in a direct and straightforward way from the formulae for the maps $f_{\alpha}$ and $f_{\beta}$. Actually it is enough to use the local formulae (4.9) for $f_{\beta}$ and (4.94) for $f_{\alpha}$, since any smooth function is determined by its restrictions to a dense open submanifold. What was non-trivial to establish is that the local formulae just mentioned represent the restrictions of global Delzant maps.

Let us discuss the meaning of Theorem 7. First note that the function $\delta \circ \mathcal{J}$ (or equivalently just $\mathcal{J})$ on $\mathbb{C} P(n-1)$ represents the global analogue of the particle-positions, since under the embedding $\mathcal{E}: M_{y}^{\text {loc }} \rightarrow \mathbb{C} P(n-1)$ we have

$$
\left(\mathcal{J}_{k} \circ \mathcal{E}\right)(\xi, \tau)=\xi_{k}
$$

According to (5.7),$f_{\beta}$ converts the reductions of the invariant functions of the form $h_{1}$, where $h_{1}(A, B)=h(A)$ before reduction, into the respective functions of the global Lax matrix $L^{y}$, and converts the reductions of the invariant functions $h_{2}$, where $h_{2}(A, B)=h(B)$ before reduction, into the respective functions of the particle-position matrix $\delta \circ \mathcal{J}$. In particular, $f_{\beta}$ converts the reduced spectral Hamiltonian $\hat{\alpha}_{k}$ into the action-variable $\Xi_{k} \circ L^{y}$ of the compactified $\mathrm{III}_{\mathrm{b}}$ system, and at the same time it converts the reduced spectral Hamiltonian $\hat{\beta}_{k}$ into the global particle-position variable $\mathcal{J}_{k}$, respectively for each $k=1, \ldots, n-1$. The formula (5.9) for the map $f_{\alpha}$ works similarly, but in addition to the exchange of the subscripts 1 and 2 on the function $\hat{h}$, which goes back to the exchange of the two factors of the double, it applies the adjoint of the unitary Lax matrix $L^{y}$ instead of $L^{y}$. This implies that, for each $k=1, \ldots, n-1, f_{\alpha}$ converts $\hat{\alpha}_{k}$ into the particle-position variable $\mathcal{J}_{k}$, and at the same time converts $\hat{\beta}_{k}$ into the "flipped action-variable' $\Xi_{n-k} \circ L^{y}$. Such a flip, which arises from the involutions inevitably involved in the relation (4.91) between $f_{\alpha}$ and $f_{\beta}$, is necessary in order to ensure the symplectic property of the map.

The phase space $\mathbb{C} P(n-1)$ of the compactified $\mathrm{III}_{\mathrm{b}}$ system is equipped with the two Abelian Poisson algebras formed by the respective functions of the form

$$
h \circ \delta \circ \mathcal{J} \text { and } h \circ L^{y} \text { with } h \in C^{\infty}\left(G_{\mathrm{reg}}\right)^{G} .
$$


These Abelian algebras are generated respectively by the global particle-position variables $\mathcal{J}_{k}$ and action-variables $\Xi_{k} \circ L^{y}$. We now describe the behaviour of these algebras under the Delzant symplectomorphism $\mathfrak{S}$,

$$
\mathfrak{S} \equiv f_{\alpha}^{-1} \circ f_{\beta}: \mathbb{C} P(n-1) \rightarrow \mathbb{C} P(n-1)
$$

as introduced in (4.117). In the following theorem, we shall use the 'flip-involution' $h \mapsto h^{\sharp}$ of $C^{\infty}\left(G_{\text {reg }}\right)^{G}$ defined by

$$
h^{\sharp}(g):=h\left(g^{\dagger}\right), \quad \forall g \in G_{\text {reg }} .
$$

With this notation, the property (4.87) of $\Xi_{k} \in C^{\infty}\left(G_{\text {reg }}\right)^{G}$ implies

$$
\Xi_{k}^{\sharp}=\Xi_{n-k}, \quad k=1, \ldots, n-1 .
$$

Theorem 8. The Delzant symplectomorphism $\mathfrak{S}$ given by (5.13) satisfies the identities

$$
(h \circ(\delta \circ \mathcal{J})) \circ \mathfrak{S}=h \circ L^{y} \quad \text { and } \quad\left(h \circ L^{y}\right) \circ \mathfrak{S}=h^{\sharp} \circ(\delta \circ \mathcal{J})
$$

for every $h \in C^{\infty}\left(G_{\mathrm{reg}}\right)^{G}$. In particular, with $h=\Xi_{k}(k=1, \ldots, n-1)$ this yields

$$
\mathcal{J}_{k} \circ \mathfrak{S}=\Xi_{k} \circ L^{y} \quad \text { and } \quad\left(\Xi_{k} \circ L^{y}\right) \circ \mathfrak{S}=\mathcal{J}_{n-k} .
$$

In this way, $\mathfrak{S}$ converts the particle-position variables into the action-variables and converts the action-variables into the flipped particle-position variables.

Proof. Both equalities in (5.16) follow by trivial one line calculations from our preceding results. In fact, by using the definition of $\mathfrak{S}$ and Theorem 7 we can write

$$
(h \circ \delta \circ \mathcal{J}) \circ \mathfrak{S}=\left(\hat{h}_{1} \circ f_{\alpha}\right) \circ\left(f_{\alpha}^{-1} \circ f_{\beta}\right)=\hat{h}_{1} \circ f_{\beta}=h \circ L^{y},
$$

and similarly

$$
\left(h \circ L^{y}\right) \circ \mathfrak{S}=\left(h^{\sharp} \circ\left(L^{y}\right)^{\dagger}\right) \circ\left(f_{\alpha}^{-1} \circ f_{\beta}\right)=\left(h^{\sharp} \circ\left(L^{y}\right)^{\dagger} \circ f_{\alpha}^{-1}\right) \circ f_{\beta}=h_{2}^{\sharp} \circ f_{\beta}=h^{\sharp} \circ(\delta \circ \mathcal{J}) .
$$

The equalities in (5.17) are special cases of those in (5.16), using also (5.15).

The message of Theorem 8 is that $\mathfrak{S}$ is the symplectic version of Ruijsenaars' self-duality map of the compactified $\mathrm{III}_{\mathrm{b}}$ system. The symplectic property of this map is an automatic consequence of our construction, while it was proved in [31] in a very different manner.

It is worth noting that, as follows immediately from Theorem 8 , the symplectomorphism $\mathfrak{S}^{2}$ satisfies the relations

$$
(h \circ(\delta \circ \mathcal{J})) \circ \mathfrak{S}^{2}=h^{\sharp} \circ(\delta \circ \mathcal{J}), \quad\left(h \circ L^{y}\right) \circ \mathfrak{S}^{2}=h^{\sharp} \circ L^{y},
$$

\footnotetext{
${ }^{10}$ To give a precise comparison, our map $\mathfrak{S}$ corresponds to $f=k \circ \phi$ in Eq. (4.125) of 31. The anti-symplectic involution $\mathfrak{R}=\hat{C} \circ \mathfrak{S}$ defined here in Eq. (4.117) corresponds to $\phi$ in [31. The involutions $\hat{C}, \hat{\Gamma}, \hat{\sigma}$ that we introduced in Subsection 4.4 correspond respectively to $k, \hat{k}, p$ in Subsection 4.4 of [31, which also contains the equivalents of the relations (4.92), (4.95) and (4.118) enjoyed by $\mathfrak{S}$ and $\mathfrak{R}$. The identification of $k$ and $p$ with time reversal and parity is further discussed in 32] (page 69).
} 


$$
\mathcal{J}_{k} \circ \mathfrak{S}^{2}=\mathcal{J}_{n-k}, \quad\left(\Xi_{k} \circ L^{y}\right) \circ \mathfrak{S}^{2}=\Xi_{n-k} \circ L^{y} .
$$

These are consistent with the fact that $\mathfrak{S}^{2}$ is equal to the symplectic involution $\hat{\sigma}$ of $\mathbb{C} P(n-1)$ according to (4.118).

Ruijsenaars focused mainly on the anti-symplectic version of his self-duality map that has somewhat simpler (but equivalent) properties as the symplectic map $\mathfrak{S}$. As we now explain, this map is provided in our setting by $\mathfrak{R} \equiv \hat{C} \circ \mathfrak{S}$, defined already in (4.117). Our map $\mathfrak{R}$ is obviously anti-symplectic, $\mathfrak{R}^{*} \omega_{\mathrm{FS}}=-\omega_{\mathrm{FS}}$, and it is involutive according to (4.118). For any $h \in C^{\infty}\left(G_{\mathrm{reg}}\right)^{G}$, we readily derive that

$$
(h \circ(\delta \circ \mathcal{J})) \circ \mathfrak{R}=h \circ L^{y}, \quad\left(h \circ L^{y}\right) \circ \mathfrak{R}=h \circ(\delta \circ \mathcal{J}),
$$

which in particular implies that

$$
\mathcal{J}_{k} \circ \mathfrak{R}=\Xi_{k} \circ L^{y} \quad \text { and } \quad\left(\Xi_{k} \circ L^{y}\right) \circ \mathfrak{R}=\mathcal{J}_{k} \quad \text { for all } \quad k=1, \ldots, n-1 .
$$

These are precisely the characteristic properties of the anti-symplectic self-duality involution of Ruijsenaars [31]. The derivation of the first relation in (5.22) goes simply as

$$
h \circ \delta \circ \mathcal{J} \circ \hat{C} \circ \mathfrak{S}=h \circ \delta \circ \mathcal{J} \circ \mathfrak{S}=h \circ L^{y},
$$

by combining (5.16) with $\mathcal{J} \circ \hat{C}=\mathcal{J}$. The second relation in (5.22) can be derived similarly using (5.16) and the equality $L^{y} \circ \hat{C}=\bar{L}^{y}$ (4.122).

The relative advantage of $\mathfrak{R}$ over $\mathfrak{S}$ is that it simply interchanges the respective action and particle-position variables, with the price that $\mathfrak{R}$ is anti-symplectic while $\mathfrak{S}$ is symplectic. The relationship between $\mathfrak{S}$ and $\mathfrak{R}$ is similar to that between the symplectic map $(q, p) \mapsto(p,-q)$ and the anti-symplectic map $(q, p) \mapsto(p, q)$ on the canonical phase space $\mathbb{R}^{2 n}$. We note that the seminal paper [31] contains further interesting properties of the maps $\mathfrak{S}, \mathfrak{R}$ and 'discrete symmetries' (including but not exhausted by the time reversal $\hat{C}$ and the parity $\hat{\sigma}$ ), which we could also reproduce. This, perhaps together with the investigation of open questions mentioned in [31, will be reported in a survey that we plan to write about the reduction approach to Ruijsenaars dualities in a few years.

\section{Duality and the mapping class group}

As was already mentioned, quasi-Hamiltonian geometry provides universal finite-dimensional 'master' objects by reductions of which one may obtain the symplectic moduli spaces of nonAbelian flat connections on Riemann surfaces with prescribed boundary conditions [1]. Originally those moduli spaces were derived by infinite-dimensional reductions of infinite-dimensional symplectic manifolds $\mathcal{A}(\Sigma)$ consisting of all (suitably smooth) non-Abelian connections on the Riemann surface $\Sigma$. If $\Sigma$ is a torus with an open disc removed (called 'one-holed torus'), then the relevant finite-dimensional master object is precisely the internally fused quasi-Hamiltonian double of the non-Abelian group.

The treatment of the compactified $\mathrm{III}_{\mathrm{b}}$ system by quasi-Hamiltonian reduction now permits us to establish the validity of a remarkable interpretation of this system that goes back to 
Gorsky and Nekrasov [14]. Namely, it is immediate from [1] and our results that the compactified Ruijsenaars-Schneider system is the natural integrable system on the moduli space of flat $S U(n)$ connections on the one-holed torus, where the holonomy around the hole is constrained to the conjugacy class of the moment map value $\mu_{0}$ that we used to define our reduction. In this picture, the pair $(A, B)$ in the double represents the holonomies of the flat connection along the standard cycles on the torus. This interpretation of the $\mathrm{III}_{\mathrm{b}}$ system was anticipated in [14] based on some formal, non-rigorous arguments. Utilizing the quasi-Hamiltonian framework, we here further develop this interpretation and clarify the connection (conjectured in [10]) between the mapping class group of the one-holed torus and the Ruijsenaars duality.

In the next two subsections 6.1 and 6.2 we briefly summarize necessary background material [1, 13], and present new results in the last two subsections 6.3 and 6.4. In the background material $G$ can be taken to be any connected and simply connected compact Lie group, with invariant scalar product $\langle$,$\rangle on its Lie algebra \mathcal{G}$, but when dealing with our example it will be understood without further notice that $G \equiv S U(n)$.

\subsection{Flat connections and the quasi-Hamiltonian double}

Let $\mathcal{A}(\Sigma)$ be the space of smooth $\mathcal{G}$-valued connection forms on the one-holed torus $\Sigma$. The space $\mathcal{A}(\Sigma)$ carries a natural symplectic form $\Omega$ with respect to which the gauge group $G(\Sigma)$ of smooth maps from $\Sigma$ to $G$ acts in the Hamiltonian way. The symplectic form reads

$$
\Omega(a, b)=\int_{\Sigma}\langle a \wedge, b\rangle
$$

where $a$ and $b$ are $\mathcal{G}$-valued 1 -forms on $\Sigma$ interpreted as tangent vectors to $\mathcal{A}(\Sigma)$. Consider then the space $\mathcal{A}_{\text {flat }}(\Sigma)$ of connections $\phi$ verifying the flatness condition $d \phi+[\phi, \phi]=0$ and the subgroup $G_{\text {res }}(\Sigma)$ of $G(\Sigma)$ mapping a chosen point $p_{0}$ on the boundary of the hole to the unit element of the group $G$. The quotient $\mathcal{A}_{\text {flat }}(\Sigma) / G_{\text {res }}(\Sigma)$ can be identified with the finite-dimensional manifold $G \times G$ because every flat connection is completely determined by its $\left(G_{\text {res }}(\Sigma)\right.$-independent) holonomies along two generators of the fundamental group $\pi_{1}\left(\Sigma, p_{0}\right)$. Furthermore, as shown in [1], the symplectic form $\Omega$ induces the quasi-Hamiltonian form (2.11) on the double $G \times G$ such that every quasi-Hamiltonian reduction of the double $(G \times G, \omega)$ gives the same outcome as a corresponding symplectic reduction of $(\mathcal{A}(\Sigma), \Omega)$. In particular, the quasi-Hamiltonian reduction based on the moment map value $\mu_{0}(3.2)$ that we have performed in Section 3 gives the symplectic manifold $P$ that can be also interpreted as the moduli space $\mathcal{A}_{\text {flat }}(\Sigma, C) / G(\Sigma)$ consisting of flat connections with holonomies around the boundary of the hole belonging to the conjugacy class $C$ of $\mu_{0}$ modulo $G(\Sigma)$ gauge transformations.

Since the concepts of quasi-Hamiltonian geometry and quasi-Hamiltonian reduction make no reference to flat connections on Riemann surfaces, the following question appears. Despite technical advantages of the quasi-Hamiltonian vantage point, are there some relevant structural insights concerning the symplectic geometry of the moduli spaces that are still more transparent in the infinite-dimensional reduction approach? The answer to this question is affirmative and this section is devoted to an important illustration of this fact. Indeed, we shall show that the symplectic version $\mathfrak{S}$ of the Ruijsenaars duality map introduced in Section 4 is nothing but the 
natural symplectomorphism induced by a particular element of the mapping class group of the one-holed torus.

Recall that the group $\operatorname{Diff}^{+}(\Sigma)$ of orientation-preserving diffeomorphisms acts symplectically on the space of all connections $(\mathcal{A}(\Sigma), \Omega)$ by simply associating to a connection 1-form $\phi$ its pull-back by the diffeomorphism. This infinite-dimensional group of symplectomorphisms has an immense 'reduction kernel' consisting of all diffeomorphisms which can be connected to the identity. This means that effectively only the corresponding discrete quotient group (known as the mapping class group) acts on the moduli space $\mathcal{A}_{\text {flat }}(\Sigma, C) / G(\Sigma)=P$. We shall explain how this symplectic action on the reduced phase space $P$ can be understood entirely in the quasiHamiltonian context as well. In fact, it turns out that an appropriate central extension of the mapping class group acts by automorphisms on the quasi-Hamiltonian double, and upon quasiHamiltonian reduction the center factors out yielding the symplectic action of the orientationpreserving mapping classes on the reduced phase space.

\subsection{Mapping class group of the one-holed torus}

The diffeomorphisms considered below include also those which reverse the orientation of the Riemann surface. We shall need this generality later on in order to explain the geometrical origin of both the symplectic version $\mathfrak{S}$ and the anti-symplectic version $\mathfrak{R}$ of the duality map.

We recall (see e.g. [13]) that the fundamental group $\pi_{1}:=\pi_{1}\left(\Sigma, p_{0}\right)$ of the one-holed torus is a free group with two generators $X$ and $Y$ corresponding to the pathes passing through the point $p_{0}$ and forming the standard homology basis of the torus. It admits a redundant presentation in terms of three generators $X, Y, K$ :

$$
\pi_{1}=\left\{X, Y, K \mid X Y X^{-1} Y^{-1}=K\right\}
$$

where $K$ corresponds to the generator of the boundary fundamental group $\pi_{1}\left(\partial \Sigma, p_{0}\right)$. Every element from the group of diffeomorphisms Diff $p_{0}(\Sigma)$ preserving the point $p_{0}$ induces an automorphism of the fundamental group $\pi_{1}$ and two diffeomorphisms from Diff $p_{0}(\Sigma)$ which can be connected by a path in $\operatorname{Diff}(\Sigma)$ induce automorphisms of $\pi_{1}$ differing by an inner automorphism. Let $\operatorname{Diff}_{p_{0}, 0}(\Sigma)$ be the normal subgroup of $\operatorname{Diff}_{p_{0}}(\Sigma)$ consisting of the elements connected to the identity by a path in $\operatorname{Diff}(\Sigma)$. The mapping class group can be defined as

$$
\mathrm{MCG}_{p_{0}}(\Sigma) \equiv \operatorname{Diff}_{p_{0}}(\Sigma) / \operatorname{Diff}_{p_{0}, 0}(\Sigma),
$$

and there exists a natural homomorphism

$$
\mathcal{N}: \operatorname{MCG}_{p_{0}}(\Sigma) \rightarrow \operatorname{Out}\left(\pi_{1}\right) \equiv \operatorname{Aut}\left(\pi_{1}\right) / \operatorname{Inn}\left(\pi_{1}\right) .
$$

Nielsen [24] has proved that $\mathcal{N}$ is actually an isomorphism identifying $\mathrm{MCG}_{p_{0}}(\Sigma)$ with $\operatorname{Out}\left(\pi_{1}\right)$. Furthermore one has the standard Hurewicz homomorphism from $\pi_{1}$ into the homology group $H_{1}(\Sigma ; \mathbb{Z})=\pi_{1} /\left[\pi_{1}, \pi_{1}\right] \cong \mathbb{Z}^{2}$. Since $H_{1}(\Sigma ; \mathbb{Z})$ is Abelian, its outer automorphisms obviously form the discrete linear group $G L(2, \mathbb{Z})$. Hence one obtains a homomorphism

$$
\mathcal{H}: \operatorname{Out}\left(\pi_{1}\right) \rightarrow G L(2, \mathbb{Z}),
$$


which again turns out to be an isomorphism [23].

In addition to the isomorphisms $\mathcal{N}$ and $\mathcal{H}$, there exists another isomorphism (see e.g. [13])

$$
\mathcal{I}: G L(2, \mathbb{Z}) \rightarrow \operatorname{Aut}\left(\pi_{1}, K\right) /\left\langle\iota_{K}\right\rangle
$$

which will be especially useful for us. Here $\operatorname{Aut}\left(\pi_{1}, K\right)$ denotes the subgroup of automorphisms of $\pi_{1}$ which take $K$ either to $K$ or to $K^{-1}$ and $\left\langle\iota_{K}\right\rangle$ is the normal subgroup of $\operatorname{Aut}\left(\pi_{1}, K\right)$ formed by conjugations by powers of $K$. The existence of the isomorphism $\mathcal{I}$ is based on the fact that the centralizer of $K$ in $\pi_{1}$ is the infinite cyclic group $\langle K\rangle$ and on the result of Nielsen [23] stating that any automorphism of $\pi_{1}$ takes $K$ to a conjugate of either $K$ itself or to a conjugate of its inverse $K^{-1}$. Notice that as the one-holed torus has just one boundary component, which is therefore preserved by every element of $\operatorname{Diff}_{p_{0}}(\Sigma)$, in the image of $\mathcal{N}$ (6.4) there may occur only such elements of Out $\left(\pi_{1}\right)$ that preserve the union of the conjugacy classes of $K$ and of $K^{-1}$ in $\pi_{1}$. The orientation-preserving mapping classes are mapped by $\mathcal{N}$ into outer automorphisms respecting the conjugacy class of $K$ alone. (For the results recalled so far, the reader may also consult the reviews [16, 19].)

By composing the isomorphisms $\mathcal{N}, \mathcal{H}$ and $\mathcal{I}$ we identify the mapping class group $\operatorname{MCG}_{p_{0}}(\Sigma)$ with the group $\operatorname{Aut}\left(\pi_{1}, K\right) /\left\langle\iota_{K}\right\rangle$. Four particular elements $F, S, T$ and $\tilde{T}$ of $\operatorname{Aut}\left(\pi_{1}, K\right)$ will feature subsequently. We define them as follows:

$$
\begin{aligned}
& F: X \rightarrow Y, \quad Y \rightarrow X ; \quad S: X \rightarrow Y^{-1}, \quad Y \rightarrow Y X Y^{-1} \\
& T: X \rightarrow X Y, \quad Y \rightarrow Y ; \quad \tilde{T}: X \rightarrow X, \quad Y \rightarrow Y X^{-1}
\end{aligned}
$$

These elements (redundantly) generate $\operatorname{Aut}\left(\pi_{1}, K\right)$ [13. Since $\mathcal{N}$ (6.4) is an isomorphism, there exist diffeomorphisms inducing the actions of their equivalence classes on $\pi_{1}$. The diffeomorphisms (and their mapping classes) that yield $[T]$ and $[\tilde{T}]$ are commonly referred to as Dehn twists around the cycles $Y$ and $X$, respectively.

By an easy calculation we find

$$
F^{2}=(F S)^{2}=(F S T)^{2}=\mathrm{Id}, \quad S^{2}=(S T)^{3}, \quad S^{4}: X \rightarrow K^{-1} X K, \quad Y \rightarrow K^{-1} Y K,
$$

and therefore the equivalence classes $[F],[S]$ and $[T]$ in $\operatorname{Aut}\left(\pi_{1}, K\right) /\left\langle\iota_{K}\right\rangle$ verify

$$
[F]^{2}=([F][S])^{2}=([F][S][T])^{2}=\mathrm{Id}, \quad[S]^{2}=([S][T])^{3}, \quad[S]^{4}=\mathrm{Id} .
$$

It is well-known ([4], Eq. (7.21)) that the relations in (6.9) are defining relations of the group $G L(2, \mathbb{Z})$ and the last two of them define the subgroup $S L(2, \mathbb{Z})$. Correspondingly, we have the identifications

$$
S L(2, \mathbb{Z}) \simeq \operatorname{Aut}^{+}\left(\pi_{1}, K\right) /\left\langle\iota_{K}\right\rangle \simeq \mathrm{MCG}_{p_{0}}^{+}(\Sigma),
$$

where $\operatorname{Aut}^{+}\left(\pi_{1}, K\right)$ is generated by $S$ and $T$ and $\mathrm{MCG}_{p_{0}}^{+}(\Sigma)$ contains the orientation-preserving mapping classes. It is helpful to display the integer matrices associated to the classes $[F],[S]$ and $[T]$ by the isomorphism $\mathcal{I}([6.6)$. Following [13], we obtain

$$
[F] \rightarrow\left[\begin{array}{ll}
0 & 1 \\
1 & 0
\end{array}\right], \quad[S] \rightarrow\left[\begin{array}{cc}
0 & 1 \\
-1 & 0
\end{array}\right], \quad[T] \rightarrow\left[\begin{array}{ll}
1 & 0 \\
1 & 1
\end{array}\right]
$$


We did not need all four automorphisms $F, S, T$ and $\tilde{T}$ to characterize the mapping class group, since the relation $\tilde{T}=(T S T)^{-1}$ is valid. Instead of $F, S$ and $T$ we can use as generators $F, T$ and $\tilde{T}$, whereby $[S]$ can be expressed as the composition of three successive Dehn twists:

$$
[S]=([T][\tilde{T}][T])^{-1} .
$$

\subsection{The duality map $\mathfrak{S}$ as a mapping class symplectomorphism}

By definition, an automorphism of a quasi-Hamiltonian space $(M, G, \omega, \mu)$ is a diffeomorphism of $M$ that preserves $\omega$ and $\mu$ and commutes with the $G$-action. We next exhibit a natural homomorphism from the group $\mathrm{Aut}^{+}\left(\pi_{1}, K\right)$ generated by $S$ and $T$ (6.7) into the group $\operatorname{Aut}(D(G))$ of automorphisms of the double. For this purpose, we set

$$
S(A, B):=\left(B^{-1}, B A B^{-1}\right), \quad T(A, B):=(A B, B), \quad \tilde{T}(A, B):=\left(A, B A^{-1}\right) .
$$

One readily verifies that the maps $S, T$ and $\tilde{T}$ defined by (6.13) preserve the basic structures (2.10), (2.11) and (2.12) of the double, giving automorphisms of $D(G)$. Note that we slightly abuse the notation by using the same symbols $S, T, \tilde{T}$ for the elements of $\operatorname{Aut}^{+}\left(\pi_{1}, K\right)$ and of $\operatorname{Aut}(D(G))$, and later we shall use them even for corresponding elements of factor groups of $\operatorname{Aut}^{+}\left(\pi_{1}, K\right)$ and of $\operatorname{Aut}(D(G))$. We believe however that the reader prefers to figure out from the context which group we have in mind rather than to remember which of several different putative notations corresponds to which group.

The similarity of the formulae (6.7) and (6.13) implies that in (6.13) we have constructed a homomorphism of $\operatorname{Aut}^{+}\left(\pi_{1}, K\right)$ into $\operatorname{Aut}(D(G))$. This similarity is not accidental, of course. Indeed, the assignment of a pair of $G$-elements $A$ and $B$ to a flat connection is not unique but it is fixed by the choice of the homology cycles along which one computes the holonomies of the flat connection. It is the standard choice of homology basis which we have adopted previously. The transition from the standard homology basis to another one following the matrices (6.11) induces the automorphisms (6.13) of the double.

As we know, the fourth power $S^{4}$ in $\operatorname{Aut}^{+}\left(\pi_{1}, K\right)$ is the conjugation by the element $K^{-1}$, which belongs to the center of $\operatorname{Aut}^{+}\left(\pi_{1}, K\right)$. On the other hand, calculating $S^{4}$ (6.13) in $\operatorname{Aut}(D(G))$ gives the so-called twist automorphism $Q$ introduced in [1]:

$$
Q(A, B)=\Psi_{\mu(A, B)^{-1}}(A, B) .
$$

It is easy to check directly that the twist automorphism is in the center of the group $\operatorname{Aut}(D(G))$ (this is by the way true for any quasi-Hamiltonian manifold). The Abelian group $\langle Q\rangle$ generated by $Q$ is thus a distinguished normal subgroup of $\operatorname{Aut}(D(G))$, and $\operatorname{Aut}(D(G))$ can be viewed as a central extension of the group $\operatorname{Aut}(D(G)) /\langle Q\rangle$. On account of (6.10), we may conclude that the orientation-preserving subgroup of the mapping class group acts on the double $D(G)$ 'projectively'.

Turning to quasi-Hamiltonian reduction, note that the automorphisms $S, T$ and $\tilde{T}$ defined in (6.13) respect the constraint surface $\mu^{-1}\left(\mu_{0}\right)$. Moreover, $\mu_{0}$ belongs to the isotropy group $G_{0}$ and therefore the automorphism $Q$ descends to the trivial identity map on the reduced phase space $P=\mu^{-1}\left(\mu_{0}\right) / G_{0}$. Thus $S, T$ and $\tilde{T}$ descended on $P$ generate a true action of the 
orientation-preserving subgroup of the mapping class group, $\mathrm{MCG}_{p_{0}}^{+}(\Sigma)$ given by (6.10). By construction, this action operates via symplectomorphisms of $(P, \hat{\omega})$.

We are now going to demonstrate that in the $\mathbb{C} P(n-1)$ parametrization of the reduced phase space $P$ of our interest the mapping class generator $S$ yields just the Ruijsenaars self-duality symplectomorphism $\mathfrak{S}$ of $\mathbb{C} P(n-1)$.

Theorem 9. With the choice $\mu_{0}$ in (3.2), let us denote by $S_{P}: P \rightarrow P$ the mapping class symplectomorphism that descends from the automorphism $S$ (6.13). Then

$$
\mathfrak{S}=f_{\beta}^{-1} \circ S_{P} \circ f_{\beta},
$$

where $f_{\beta}: \mathbb{C} P(n-1) \rightarrow P$ is the Delzant symplectomorphism constructed in Section 4 and $\mathfrak{S}: \mathbb{C} P(n-1) \rightarrow \mathbb{C} P(n-1)$ is the Ruijsenaars symplectomorphism defined in (4.117).

Proof. We have obviously

$$
\left(S_{P} \circ p\right)(A, B)=p\left(B^{-1}, B A B^{-1}\right), \quad \forall(A, B) \in \mu^{-1}\left(\mu_{0}\right),
$$

where $p$ is the projection from $\mu^{-1}\left(\mu_{0}\right)$ to $P=\mu^{-1}\left(\mu_{0}\right) / G_{0}$. Recall the involution $\hat{\nu}$ that verifies $(\hat{\nu} \circ p)(A, B)=p(\bar{B}, \bar{A})$ and descends from the map $\nu(A, B)=(\bar{B}, \bar{A})$ defined on the double in (4.82). We can thus write

$$
\left(\hat{\nu} \circ S_{P} \circ p\right)(A, B)=p\left(\bar{B} \bar{A} \bar{B}^{-1}, \bar{B}^{-1}\right), \quad \forall(A, B) \in \mu^{-1}\left(\mu_{0}\right) .
$$

Let us show that

$$
\hat{\nu} \circ S_{P} \circ f_{\beta}=f_{\beta} \circ \hat{C},
$$

where $\hat{C}$ is the complex conjugation on $\mathbb{C} P(n-1)$ introduced in (4.85). By continuity, it is sufficient to verify (6.18) on $\mathbb{C} P(n-1)_{0}$, where (thanks to the formula (4.9)) it can be rewritten as the equality

$$
p \circ \Psi_{g_{y}(\xi)^{-1}}\left(\delta(\xi)^{-1} L_{y}^{\mathrm{loc}}\left(\delta(\xi), \rho(\tau)^{-1}\right)^{*} \delta(\xi), \delta(\xi)\right)=p \circ \Psi_{g_{y}(\xi)^{-1}}\left(L_{y}^{\mathrm{loc}}(\delta(\xi), \rho(\tau)), \delta(\xi)\right),
$$

for all $(\xi, \tau) \in \mathcal{P}_{y}^{0} \times \mathbb{T}_{n-1}$. But this holds simply on account of equation (4.112) applied to $-y$ instead of $y$.

Now we use the involutivity of $\hat{\nu}$, then Theorem 6 , which states that $f_{\alpha}=\hat{\nu} \circ f_{\beta} \circ \hat{\Gamma}$, then also equation (4.119), rewritten as $\mathfrak{S}^{2}=\hat{\Gamma} \circ \hat{C}$, and the definition of the map $\mathfrak{S}:=f_{\alpha}^{-1} \circ f_{\beta}$ (4.117) to conclude from (6.18)

$$
f_{\beta}^{-1} \circ S_{P} \circ f_{\beta}=f_{\beta}^{-1} \circ \hat{\nu} \circ f_{\beta} \circ \hat{C}=f_{\beta}^{-1} \circ f_{\alpha} \circ \hat{\Gamma} \circ \hat{C}=f_{\beta}^{-1} \circ f_{\alpha} \circ \mathfrak{S}^{2}=\mathfrak{S}^{-1} \circ \mathfrak{S}^{2}=\mathfrak{S} .
$$

As is well-known, and is evident from (6.12), the Dehn twists $T$ and $\tilde{T}$ can be used as alternative generators of the (orientation-preserving) mapping class group instead of $T$ and $S$. This directly leads to the decomposition

$$
S_{P}=\left(T_{P} \circ \tilde{T}_{P} \circ T_{P}\right)^{-1}
$$


Applying ideas of Goldman [12] (see also [21]), we now show that the Dehn twist symplectomorphisms $T_{P}$ and $\tilde{T}_{P}$ themselves are specializations of simple Hamiltonian flows on $P$. More precisely, we can realize already the automorphisms $T$ and $\tilde{T}$ of $D$ given in (6.13) by means of quasi-Hamiltonian flows as stated by the following lemma.

Lemma 11. Employing the notations of Subsection 2.2, define the functions $h \in C^{\infty}\left(D_{b}\right)^{G}$ and $\tilde{h} \in C^{\infty}\left(D_{a}\right)^{G}$ by

$$
h=\operatorname{tr}\left(\sum_{k=1}^{n-1} \beta_{k} \lambda_{k}\right)^{2}, \quad \tilde{h}=\operatorname{tr}\left(\sum_{k=1}^{n-1} \alpha_{k} \lambda_{k}\right)^{2},
$$

and let $\phi_{h, s}$ and $\phi_{\tilde{h}, s}$ be the corresponding quasi-Hamiltonian flows. Then, respectively on $D_{b}$ and on $D_{a}$, there hold the equalities $T(A, B)=\phi_{h, 1}(A, B)$ and $\tilde{T}(A, B)=\phi_{\tilde{h}, 1}(A, B)$.

Proof. Using the definitions of Subsection 2.2, introduce arbitrary real powers of any $C \in G_{\text {reg }}$ by setting

$$
C^{s}:=g(C)^{-1} \exp \left(-2 \mathrm{i} s \sum_{k=1}^{n-1} \Xi_{k}(C) \lambda_{k}\right) g(C), \quad \forall s \in \mathbb{R} .
$$

Then it follows from equations (2.15), (2.16) and (2.24) that

$$
\phi_{h, s}(A, B)=\left(A B^{s}, B\right), \quad \phi_{\tilde{h}, s}(A, B)=\left(A, B A^{-s}\right),
$$

and comparison with (6.13) entails the claim.

The functions $h$ and $\tilde{h}$ descend to $P$, and when transferred to the model $\mathbb{C} P(n-1)$ they become functions of the global particle-positions, $\hat{\beta}_{k} \circ f_{\beta}$, and action-variables, $\hat{\alpha}_{k} \circ f_{\beta}$. The decomposition of the Ruijsenaars duality map $\mathfrak{S}$ implied by (6.15) and (6.21) represents a new result. This is a simple by-product of the reduction approach, which would have been difficult to notice in the direct approach [31] to the compactified $\mathrm{III}_{\mathrm{b}}$ system.

\subsection{The anti-symplectomorphism $\mathfrak{R}$ as a $G L(2, \mathbb{Z})$ generator}

In Section 6.3, we have implemented the generators $S$ and $T$ of the orientation-preserving $S L(2, \mathbb{Z})$ part of the full mapping class group $G L(2, \mathbb{Z})$ as automorphisms of the double that descend upon reduction to the symplectomorphisms $S_{P}$ and $T_{P}$ of our reduced phase space $P$. We now observe that, on the one hand, the third generator $[F]$ of $G L(2, \mathbb{Z})$ implemented, according to (6.7), as the map $F_{D}(A, B):=(B, A)$ is not an automorphism of the double and it does not survive the quasi-Hamiltonian reduction. On the other hand, if we consider instead of $F_{D}$ the related map $\nu: D(G) \rightarrow D(G)$ defined by (4.82) as $\nu(A, B)=(\bar{B}, \bar{A})$, then $\nu$ maps $\mu^{-1}\left(\mu_{0}\right)$ to itself and induces the anti-symplectic involution $\hat{\nu}$ of the reduced phase space. Moreover, it is readily checked that under the assignment

$$
[F] \mapsto \hat{\nu}, \quad[S] \mapsto S_{P}, \quad[T] \mapsto T_{P}
$$

$\hat{\nu}, S_{P}$ and $T_{P}$ fulfil the generating relations (6.9) of $G L(2, \mathbb{Z})$, and thus they induce an action of $G L(2, \mathbb{Z})$ on $P$. Note also that $G L(2, \mathbb{Z})$ can be written as the semi-direct product

$$
G L(2, \mathbb{Z})=\mathbb{Z}_{2} \ltimes S L(2, \mathbb{Z}),
$$


where the $\mathbb{Z}_{2}$ subgroup is generated by $[F]$. Correspondingly, there are two kinds of elements of $G L(2, \mathbb{Z}):(+, \rho)$ and $(-, \rho)$ where $\rho \in S L(2, \mathbb{Z})$ and \pm is the sign of the determinant of the $G L(2, \mathbb{Z})$ matrix. The elements $(+, \rho)$ acting on $P$ are symplectomorpisms and $(-, \rho)$ are anti-symplectomorphisms. This follows from the fact that $\hat{\nu} \equiv(-, e)$ reverses the sign of the symplectic form on $P$ (since $\nu$ reverses the sign of $\omega$ on $D(G)$ ) while $S_{P}$ and $T_{P}$ preserve it.

Parametrizing our reduced phase space $P$ as $\mathbb{C} P(n-1)$ by means of the Delzant symplectomorphism $f_{\beta}$, as before, the generator $\hat{\nu}$ of $G L(2, \mathbb{Z})$ becomes $f_{\beta}^{-1} \circ \hat{\nu} \circ f_{\beta}$ and it is directly related to the involutive Ruijsenaars anti-symplectomorphism $\mathfrak{R}: \mathbb{C} P(n-1) \rightarrow \mathbb{C} P(n-1)$. Indeed, we find the following identities by combining Theorem 6 , which states that $f_{\alpha}=\hat{\nu} \circ f_{\beta} \circ \hat{\Gamma}$, the definition of the map $\mathfrak{S}:=f_{\alpha}^{-1} \circ f_{\beta}$ (4.117) and Eq. (4.120) saying that $\mathfrak{S} \circ \hat{\Gamma}=\mathfrak{R}$ :

$$
\mathfrak{R}=\mathfrak{S} \circ \hat{\Gamma}=f_{\alpha}^{-1} \circ f_{\beta} \circ \hat{\Gamma}=f_{\alpha}^{-1} \circ \hat{\nu} \circ f_{\alpha}=\mathfrak{S} \circ f_{\beta}^{-1} \circ \hat{\nu} \circ f_{\beta} \circ \mathfrak{S}^{-1} .
$$

Hence, the Ruijsenaars map $\mathfrak{R}$ can be viewed as an alternative anti-symplectic generator of the $G L(2, \mathbb{Z})$ action on $\mathbb{C} P(n-1)$.

We have exhibited an (anti)-symplectic action of the full group $G L(2, \mathbb{Z})$ on our reduced phase space. This action does not descend from the (projective) action of the full mapping class group on the double since we have replaced the generator $F_{D}$ by the new generator $\nu$. It is thus natural to ask the following question: Does the $G L(2, \mathbb{Z})$ generator $\hat{\nu}$ has its origin in some natural (anti)-symplectomorphism of the space of connections $(\mathcal{A}(\Sigma), \Omega)$ ? It turns out that the answer to this question is positive. Indeed, take any orientation-reversing diffeomorphism of $\Sigma$ which is in the class $F$ of the mapping class group and compose it with the complex conjugation acting on the su(n)-valued connection 1-forms (without touching their argument). Since the complex conjugation is an automorphism of the group $S U(n)$ and of its Lie algebra, this composed map is an anti-symplectomorphism of $(\mathcal{A}(\Sigma), \Omega)$ which descends to the involutive anti-symplectomorphism $\hat{\nu}$.

\section{Discussion}

In this paper we have demonstrated that an appropriate quasi-Hamiltonian reduction [1] of the internally fused double $D=S U(n) \times S U(n)$ yields a reduced phase space $P$ that turns into a Hamiltonian toric manifold (i.e. a compact completely integrable system) in two different but equivariantly symplectomorphic ways. The underlying two toric moment maps on $P$, with respective components $\hat{\alpha}_{k}$ and $\hat{\beta}_{k}$, arise from the reductions of the two sets of spectral Hamiltonians on $D$ generated by the two components of the pair $(A, B) \in D$. On the other hand, the phase space $\mathbb{C} P(n-1)$ also carries two distinguished toric structures, with moment maps $\mathcal{J}_{k}$ and $\Xi_{k} \circ L^{y}$ that encode, respectively, the particle-positions and the action-variables of the compactified $\mathrm{III}_{\mathrm{b}}$ system as discovered in [31]. We have explicitly constructed two 'Delzant symplectomorphisms' $f_{\alpha}$ and $f_{\beta}$ from $\mathbb{C} P(n-1)$ to $P$ that relate these toric moment maps according to Eqs. (5.8) and (5.10), and have identified the composed map $\mathfrak{S}=f_{\alpha}^{-1} \circ f_{\beta}$ as the symplectic self-duality map [31] of the compactified $\mathrm{III}_{\mathrm{b}}$ system, which satisfies Eq. (5.17). In our setting the symplectic property of the pertinent self-duality map is obvious, while in the original approach of 31] it required a special proof. We have also recovered the anti-symplectic version $\mathfrak{R}=\hat{C} \circ \mathfrak{S}$ of Ruijsenaars' self-duality map, which satisfies Eq. (5.23). 
In addition, we have rigorously established the interpretation of the compactified $\mathrm{III}_{\mathrm{b}}$ system in terms of flat $S U(n)$ connections on the one-holed torus suggested by Gorsky and Nekrasov [14] and, by proving the formula $\mathfrak{S}=f_{\beta}^{-1} \circ S_{P} \circ f_{\beta}$ (6.15), we have demonstrated that the Ruijsenaars self-duality map $\mathfrak{S}$ represents the natural action of the mapping class generator $S \in S L(2, \mathbb{Z})$ on $\mathbb{C} P(n-1) \simeq P$. As for the map $\mathfrak{R}$, we have shown that it arises from a $G L(2, \mathbb{Z})$ extension of the $S L(2, \mathbb{Z})$ mapping class group action on our reduced phase space.

The interpretation of the Ruijsenaars self-duality as the reduction remnant of the $S L(2, \mathbb{Z})$ mapping class generator $S$ is a long-expected result that we finally succeeded to prove thanks to the quasi-Hamiltonian technique. For the sake of objectivity, we should mention that Gorsky and his collaborators were very close to establish this interpretation; formula (4.31) of their paper [10] coincides essentially with our formula (6.13). However, they remarked that their definition $(4.31)$ of $S$ violates the $S L(2, \mathbb{Z})$ relations and they could recover a true $S L(2, \mathbb{Z})$ action only for the rational Calogero limiting case of the reduced system [10]. Since they have not furnished more quantitative details we cannot extract from their paper the precise cause of the trouble, but we believe that it may be related to the fact that our formula (6.13) also defines only the action of a suitable central extension of $S L(2, \mathbb{Z})$ on the double and not a true $S L(2, \mathbb{Z})$ action. Our point is, however, that upon the quasi-Hamiltonian reduction this projective action descends to a true $S L(2, \mathbb{Z})$ action on the reduced phase space.

Besides the coupling constant, $y$, a second parameter, $\Lambda$, can be introduced into the $\mathrm{III}_{\mathrm{b}}$ system by replacing the symplectic form (1.3) by $\Omega_{\Lambda}^{\text {loc }}:=\Lambda \Omega^{\text {loc }}$. The local Darboux variables $p_{j}$ and $x_{j}$ then parametrize $\delta$ and $\Theta$ in (1.2) as $\delta_{j}=e^{2 \mathrm{i} x_{j} / \Lambda}$ and $\Theta_{j}=e^{-\mathrm{i} p_{j}}$, whereby $x_{j}$ becomes $x_{j} / \Lambda$ also in the Hamiltonian (1.1). The parameter $\Lambda$ can be encoded in the reduction approach by choosing the invariant scalar product on $s u(n)$ to be $-\frac{\Lambda}{2} \operatorname{tr}$, which scales the 2 -form $\omega$ (2.11) as well as the reduced symplectic form and the corresponding toric moment polytope. Being a mere scale parameter of the symplectic structure, $\Lambda$ essentially plays no role at the classical level, and we omitted it to simplify the notations. However, this parameter is important at the quantum level (see [35]).

After the present paper, just one from the list of the known Ruijsenaars dualities remains to be derived in the reduction approach. It is the self-duality of the hyperbolic RuijsenaarsSchneider system for which a suitable 'double' to be reduced is still to be discovered. It may appear tempting to search for distinct real forms of the complex holomorphic constructions of [11, 26], but this scenario does not seem to work and the problem is wide open. An intriguing reformulation of the problem is to enquire whether the known self-duality map of the hyperbolic system [29] can be factorized similarly to the representation $\mathfrak{S}=f_{\alpha}^{-1} \circ f_{\beta}$ that we obtained here in the case of the compactified III $_{\mathrm{b}}$ system.

Another interesting problem for the future is to study the Ruijsenaars duality in relation to root systems different from $A_{n}$. For progress in this direction, we refer to the paper [28]. 
Acknowledgements. We wish to thank A. Alekseev for relevant remarks and for bringing reference [20] to our attention, and are also grateful to J. Huebschmann for useful information on moduli spaces. We are indebted to V. Fock, who in relation to an earlier draft posed us a question about the role of the mapping class group, which motivated our research described in Subsections 6.3 and 6.4. We thank the referee for suggesting the simple proof of the free action of $\bar{G}_{0}$, which prompted us to present here a short and rather general proof of Theorem 1 . This work was supported in part by the Hungarian Scientific Research Fund (OTKA, K77400).

\section{References}

[1] Alekseev, A., Malkin, A., Meinrenken, E.: Lie group valued moment maps. J. Differential Geom. 48, 445-495 (1998)

[2] Audin, M., Cannas da Silva, A., Lerman, E.: Symplectic Geometry of Integrable Hamiltonian Systems. Basel: Birkhäuser, 2003

[3] Calogero, F.: Solution of the one-dimensional $N$-body problem with quadratic and/or inversely quadratic pair potentials. J. Math. Phys. 12, 419-436 (1971)

[4] Coxeter, H.S.M., Moser, W.O.J.: Generators and relations for discrete groups. 3d ed., Berlin-Heidelberg-New York: Springer, 1972

[5] Delzant, T.: Hamiltoniens périodiques et images convexes de l'application moment. Bull. Soc. math. France 116, 315-339 (1988)

[6] Duistermaat, J.J., Grünbaum, F.A.: Differential equations in the spectral parameter. Commun. Math. Phys. 103, 177-240 (1986)

[7] Fehér, L., Ayadi, V.: Trigonometric Sutherland systems and their Ruijsenaars duals from symplectic reduction. J. Math. Phys. 51, 103511 (2010)

[8] Fehér, L., Klimčík, C.: On the duality between the hyperbolic Sutherland and the rational Ruijsenaars-Schneider models. J. Phys. A: Math. Theor. 42, 185202 (2009)

[9] Fehér, L., Klimčík, C.: Poisson-Lie interpretation of trigonometric Ruijsenaars duality. Commun. Math. Phys. 301, 55-104 (2011)

[10] Fock, V., Gorsky, A., Nekrasov, N., Rubtsov, V.: Duality in integrable systems and gauge theories. JHEP 07, 028 (2000)

[11] Fock, V.V., Rosly, A.A.: Poisson structure on moduli of flat connections on Riemann surfaces and the $r$-matrix. In: Moscow Seminar in Mathematical Physics, AMS Transl. Ser. 2, Vol. 191, Providence, RI: Amer. Math. Soc., 1999, pp. 67-86

[12] Goldman, W.: Invariant functions on Lie groups and Hamiltonian flows of surface group representations. Invent. Math. 85, 263-302 (1986) 
[13] Goldman, W.: The modular group action on real $S L(2)$-characters of a one-holed torus. Geometry and Topology 7, 443-486 (2003)

[14] Gorsky, A., Nekrasov, N.: Relativistic Calogero-Moser model as gauged WZW theory. Nucl. Phys. B 436, 582-608 (1995)

[15] Gotô, M.: A theorem on compact semi-simple groups. Journ. Math. Soc. Japan 1, 270-272 (1949)

[16] Ivanov, N.V.: Mapping class groups. In: Handbook of Geometric Topology, Amsterdam: Elsevier, 2002, pp. 523-633

[17] Kazhdan, D., Kostant, B., Sternberg, S.: Hamiltonian group actions and dynamical systems of Calogero type. Comm. Pure Appl. Math. XXXI, 481-507 (1978)

[18] Khesin, B., Wendt, R.: The Geometry of Infinite-Dimensional Groups. Berlin-HeidelbergNew York: Springer, 2009

[19] Lyndon, R.C., Schupp, P.E.: Combinatorial Group Theory. Berlin-Heidelberg-New York: Springer, 1977

[20] Meinrenken, E., Woodward, C.: Hamiltonian loop group actions and Verlinde factorization. J. Differential Geom. 50, 417-469 (1998)

[21] Meusburger, C.: Dual generators of the fundamental group and the moduli space of flat connections. J. Phys. A 39, 14781-14832 (2006)

[22] Moser, J.: Three integrable Hamiltonian systems connected with isospectral deformations. Adv. Math. 16, 197-220 (1975)

[23] Nielsen, J.: Die Isomorphismen der allgemeinen unendlichen Gruppe mit zwei Erzeugenden. Math. Ann. 71, 385-397 (1918)

[24] Nielsen, J.: Untersuchungen zur Topologie der geschlossenen zweiseitigen Flächen I. Acta. Math. 50, 189-358 (1927)

[25] Nijhoff, F.W., Kuznetsov, V.B., Sklyanin, E.K., Ragnisco, O.: Dynamical $r$-matrix for the elliptic Ruijsenaars-Schneider system. J. Phys. A: Math. Gen. 29, L333-L340 (1996)

[26] Oblomkov, A.: Double affine Hecke algebras and Calogero-Moser spaces. Represent. Theory 8, 243-266 (2004)

[27] Olshanetsky, M.A., Perelomov, A.M.: Explicit solution of the Calogero model in the classical case and geodesic flows on symmetric spaces of zero curvature. Lett. Nouvo Cim. 16, 333-339 (1976)

[28] Pusztai, B.G.: Action-angle duality between the $C(n)$-type hyperbolic Sutherland and the rational Ruijsenaars-Schneider-van Diejen models. Nucl. Phys. B 853, 139-173 (2011) 
[29] Ruijsenaars, S.N.M.: Action-angle maps and scattering theory for some finite-dimensional integrable systems I. The pure soliton case. Commun. Math. Phys. 115, 127-165 (1988)

[30] Ruijsenaars, S.N.M.: Finite-dimensional soliton systems. In: Integrable and Superintegrable Systems, ed. Kupershmidt, B., Singapore: World Scientific, 1990, pp. 165-206

[31] Ruijsenaars, S.N.M.: Action-angle maps and scattering theory for some finite-dimensional integrable systems III. Sutherland type systems and their duals. Publ. RIMS 31, 247-353 (1995)

[32] Ruijsenaars, S.N.M.: Systems of Calogero-Moser type. In: Proceedings of the 1994 CRMBanff Summer School 'Particles and Fields', Berlin-Heidelberg-New York: Springer, 1999, pp. 251-352

[33] Ruijsenaars, S.N.M., Schneider, H.: A new class of integrable models and their relation to solitons. Ann. Phys. (N.Y.) 170, 370-405 (1986)

[34] Sutherland, B.: Exact results for a quantum many-body problem in one dimension. Phys. Rev. A4, 2019-2021 (1971)

[35] van Diejen, J.F., Vinet, L.: The quantum dynamics of the compactified trigonometric Ruijsenaars-Schneider model. Commun. Math. Phys. 197, 33-74 (1998) 